\title{
Dessins d'enfants, Seiberg-Witten curves and conformal blocks
}

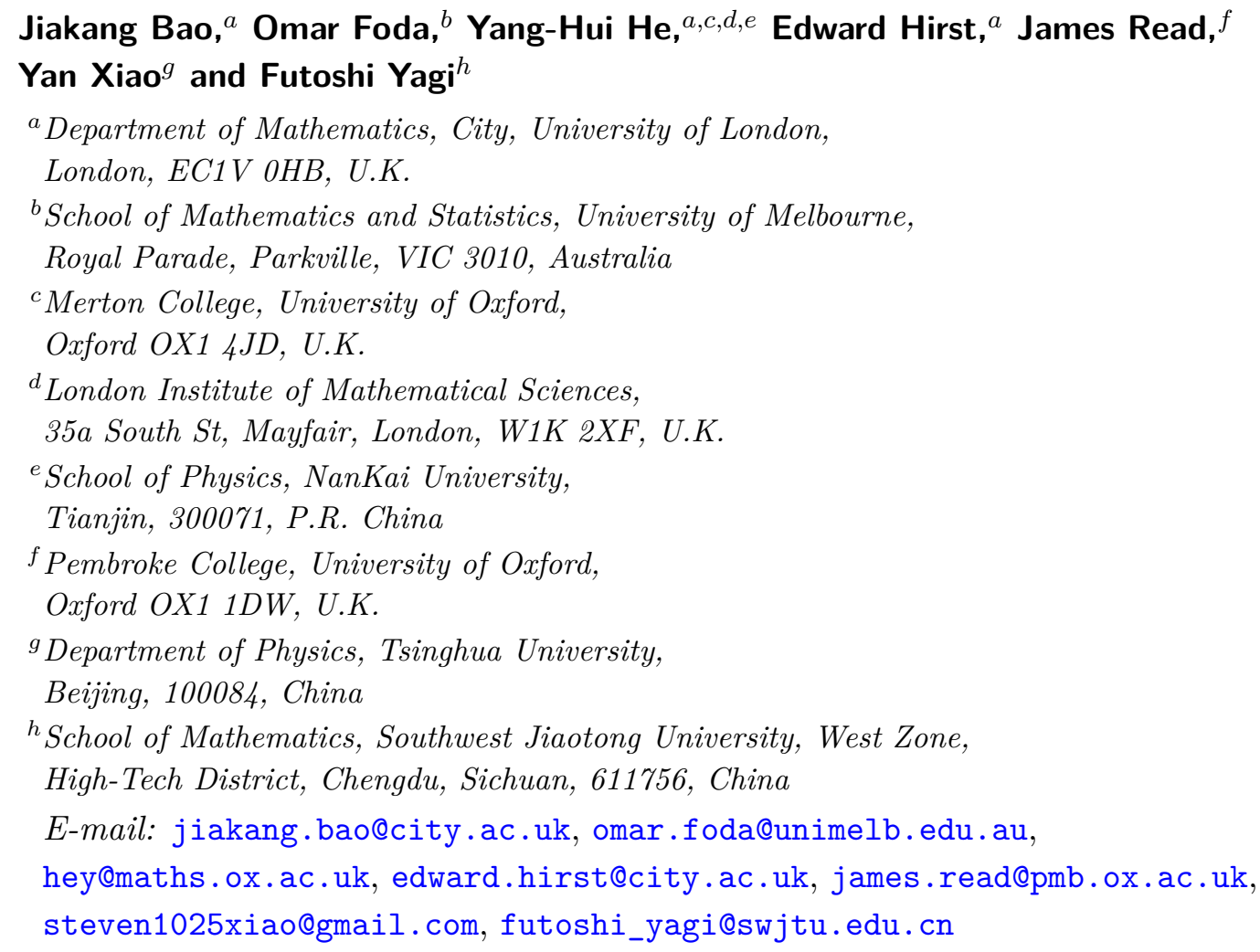

ABSTRACT: We show how to map Grothendieck's dessins d'enfants to algebraic curves as Seiberg-Witten curves, then use the mirror map and the AGT map to obtain the corresponding $4 \mathrm{~d} \mathcal{N}=2$ supersymmetric instanton partition functions and $2 \mathrm{~d}$ Virasoro conformal blocks. We explicitly demonstrate the 6 trivalent dessins with 4 punctures on the sphere. We find that the parametrizations obtained from a dessin should be related by certain duality for gauge theories. Then we will discuss that some dessins could correspond to conformal blocks satisfying certain rules in different minimal models.

Keywords: Conformal Field Theory, Differential and Algebraic Geometry, Supersymmetric Gauge Theory

ArXIV EPRINT: 2101.08843

Dedicated to the memory of our dear friend,Professor Omar Foda, a gentleman and a scholar ... 


\section{Contents}

1 Introduction and summary 1

2 From conformal blocks to dessins d'enfants 3

2.1 From 2d conformal blocks to 4d instanton partition functions 4

2.2 From $4 \mathrm{~d}$ to $5 \mathrm{~d}$ instanton partition functions and A-model topological string partition functions

2.3 From topological string partition functions to Seiberg-Witten curves 9

$\begin{array}{lll}2.4 & \text { From Seiberg-Witten curves to dessins d'enfants } & 12\end{array}$

3 From dessins to conformal blocks $\quad \mathbf{1 5}$

$\begin{array}{ll}3.1 \text { The } \mathrm{SU}(2) \text { with } 4 \text { flavours } & 15\end{array}$

$\begin{array}{lll}3.2 & \text { Example: } \Gamma(3) & 18\end{array}$

$\begin{array}{lll}3.3 & \text { Matching parameters } & 21\end{array}$

3.4 Minimal models and $\Gamma(3) \quad 26$

3.5 Minimal models and $\Gamma_{0}(4) \cap \Gamma(2) \quad 32$

3.6 Minimal models and general dessins 34

A The B-model and omega deformations $\quad 36$

$\begin{array}{ll}\text { B Brane configurations } & 37\end{array}$

$\begin{array}{lll}\text { B.1 The type IIA brane configuration } & 37\end{array}$

$\begin{array}{ll}\text { B.2 The M-theory brane configuration } & 38\end{array}$

$\begin{array}{ll}\text { C Congruence subgroups of the modular group } & 39\end{array}$

$\begin{array}{ll}\text { D Elliptic curves and } j \text {-invariants } & 40\end{array}$

E Elliptic functions and Coulomb moduli $\quad 41$

E.1 The elliptic integral of first kind 41

E.2 The elliptic logarithm 42

\section{Introduction and summary}

Consider a 4-point conformal block (CB) in a $2 \mathrm{~d}$ conformal field theory (CFT) based on $\mathcal{W}_{2} \times \mathcal{H}$, where $\mathcal{W}_{2}$ is the Virasoro algebra and $\mathcal{H}$ is the Heisenberg algebra. Using the Alday-Gaiotto-Tachikawa (AGT) correspondence [1], this is identified with an instanton partition function in an $\mathcal{N}=2$ supersymmetric Yang-Mills (SYM) theory, with an SU(2) gauge group and four fundamental hypers. 
The low energy physics of this gauge theory is described in terms of a Seiberg-Witten (SW) curve and the SW differential on it [2,3]. Then in [4], a method of instanton counting was introduced to find these low energy solutions to SW theories. Later, the S-duality for $\mathcal{N}=2$ supersymmetric systems was studied in [5]. In recent works [6-12], connections between Grothendieck's dessins d'enfants on the one hand and $4 \mathrm{~d} \mathcal{N}=2 \mathrm{SYM}$ on the other were studied. In this note, we further explore these connections and extend them to $2 \mathrm{~d}$ conformal field theory. We focus on six specific trivalent dessins with 4 punctures on the sphere, which, as we will see, are related to a simple and important class of $4 \mathrm{~d} \mathcal{N}=2$ SYM theories and conformal blocks in $2 \mathrm{~d}$ conformal field theory.

From these dessins, we obtain algebraic curves that we interpret as SW curves of $4 \mathrm{~d}$ $\mathrm{SU}(2) \mathcal{N}=2 N_{f}=4$, SYM theories. These curves are given in terms of six parameters, four mass parameters $\left(\mu_{1}, \mu_{2}, \mu_{3}, \mu_{4}\right)$, a parameter $\zeta$ and a modulus $U$. We write these curves in the form that appears in $[13,14]$, and use their mirror map to translate the above parameters to those characterizing the $4 \mathrm{~d}$ instanton partition function of a $4 \mathrm{~d} \mathcal{N}=2$ gauge theory. In particular, we map the modulus $U$ to the Coulomb parameter $a$. Following that, we use the AGT dictionary to interpret the result in 2d CFT terms.

Let us take a closer look at the six parameters for the $\mathrm{SU}(2)$ gauge theory. With $N_{f}=4$, the theory has an $\mathrm{SO}(8) \supset \mathrm{SU}(2)^{4}$ flavour symmetry. Then the mass parameters of the four hypers could be indentified as the charges of the primaries in Liouville theory under AGT correspondence [1]. Following [15, 16], the AGT map could also lead to diagonal minimal models by further restrictions on the partition pairs. As usual, we would arrange the poles of the SW curves at $z=0,1, \infty$ and $\zeta$. This $\zeta$ is nothing but the UV gauge coupling $\tau$ via $\zeta=\mathrm{e}^{2 \pi i \tau}$. For each dessin, we find that $\zeta$ could have several different values but these values enjoy certain triality.

Recall that the Coulomb parameter $a$ denotes the vev of the adjoint scalar $\phi$, or equivalently, a could be obtained by integrating the SW differential along the so-called $A$-cycle on SW curve. Such supersymmetric vacua can be gauge invariantly parametrized by $u=\left\langle\operatorname{tr} \phi^{2}\right\rangle / 2=a^{2}$ up to quantum corrections. As we will discuss in section 2.3, the parameter $U$, which will appear in the parametrization of the curve, is linear in the Coulomb moduli $u$. In fact, as we will see, each dessin gives a family of solutions for the gauge theory parameters, and indeed, we would have the same corresponding dessin under the change $m_{i} \rightarrow k m_{i}, a \rightarrow k a, U \rightarrow k^{2} U$ for $k^{2} \in \mathbb{R}$. This is consistent with their mass dimensions.

The above discussions can go the other way as well. Starting from the CBs in CFTs, we can write down the Nekrasov partition functions under the AGT dictionary. This $4 \mathrm{~d}$ partition function can also be lifted to $5 \mathrm{~d}$, which leads to topological string partition functions and SW curves. As the SW differential and the Strebel differential from the dessin side are both quadratic, the gauge theories are naturally related to dessins.

Since the instanton partition functions with extra conditions on the Young tableaux pairs could be mapped to conformal blocks in minimal models [15, 16], we can then check whether (the parametrizations from) the dessins could correspond to such CBs in minimal models. As we will see in sections 3.4-3.6, such map is not one-to-one. A dessin could correspond to one or more possible CBs in multiple minimal models. These CBs, albeit in 
different minimal models, would satisfy certain (fixed) rules for the dessin. There might also exist dessins that do not give rise to minimal models. As we are focusing on $\mathrm{SU}(2)$ gauge theory with 4 flavours, we will show that

Proposition 1.1. There is a subset of trivalent dessins with four punctures on the sphere such that each dessin therein corresponds to the (external and internal) states of a family of 4-point conformal blocks for (A-series) minimal models.

As we will discuss in section 3.6, we may also conjecture that these dessins would contain the full information for certain CBs. In principle, there are countably infinite such dessins although only a small part of them have been studied in details. As we will show, not all the dessins would give minimal models. For those in the above subset, we will also determine the families of CBs they correspond to. In particular, we will illustrate this proposition with six well-known dessins as examples. Notice that we can only say that this correspondence is not for all dessins. On the minimal model side, it is still not determined whether this subset of dessins could recover all the CBs or just part of them.

Now that the dessins are rigid, it would be interesting to understand whether the parametrizations from these dessins are special for gauge theories and (R)CFTs in future. It would also be natural to extend this for dessins with more faces and other gauge theories. So far, we only get the values for $\zeta$ from the dessins which are related by triality as briefly mentioned above, and the calculations should be non-perturbative. More details are worth studying for future works. It would be nice to even know more about the bulk of AdS through holographic duality. On the other hand, we would like to know whether the physical theories could in turn help us learn more about the dessins. For example, we know that not all dessins can give CBs in minimal models, but what kind of dessins have this correspondence is still unknown. Moreover, the Gorenthedieck-Teichmuller group, which is related to the Galois group $\Gamma(\overline{\mathbb{Q}}: \mathbb{Q})$ and dessins, may be related to the monodromy of CBs in RCFT [17]. This might lead to deeper connections between the mathematics and the physics.

The paper is outlined as follows. In section 2, we start from the CFT side and review the AGT correspondence to get the corresponding partition functions. Then from A-model topological strings, we obtain the SW curve for SU(2) with 4 flavours and thence the dessins. In section 3, we reverse the discussion and contemplate six of the dessins which would yield specific parametrizations for SW curves. Then we will study if these parametrizations would give conformal blocks in minimal models following the AGT dictionary. In the appendices, we give some background on brane systems as well as elliptic curves and elliptic functions.

This work is dedicated to the memory of Professor Omar Foda, who was instrumental in initiating this project.

\section{From conformal blocks to dessins d'enfants}

Before we derive the results in 2d CFT from the 6 dessins with 4 punctures on the sphere, we give a brief review of different subjects including CBs, topological strings, SW curves and dessins, following a route map from CBs to dessins. 


\subsection{From 2d conformal blocks to $4 d$ instanton partition functions}

The connection between 2d Liouville CFTs and $\mathrm{SU}(2)$ supersymmetric gauge theories in $4 \mathrm{~d}$ with $\mathcal{N}=2$ was first raised in [1]. The free parameters of the two areas are naturally mapped to each other under AGT correspondence. Later in $[15,16]$, the AGT correspondence was also extended to minimal models by further restrictions on the partitions/Young diagrams. We first start with the CFT side under the Coulomb-gas formalism.

Conformal blocks. Conformal blocks form a basis of the vertex operator (VO) algebra, used when performing a particular operator product expansion (OPE) of a correlation function. They are a key ingredient in the conformal bootstrap approach to calculating these correlators in 2d CFTs. Global conformal Ward identities of the CFT allow 2-point functions to be completely determined, whilst 3-point functions to have fixed results up to their respective structure constants. Thus when calculating an $N \geq 4$-point function the recursion of applying OPEs allows expression of the correlator in terms of these simpler 3-point function structure constants, and conformal blocks.

More specifically, an OPE amounts to summation over all representations of the vertex operator algebra. In the common case where this algebra factorises into two Virasoro algebras the sum includes all combinations of the left and right Virasoro algebras' representations for the CFT. Each term in the sum has a product of two conformal blocks, one in each of the term's left and right representations respectively.

Conformal blocks in general are sums over the states in their representation, they're functions of the fields' positions \& conformal dimensions, the conformal dimension of the basis expansion, and the central charge of the algebra. Blocks over primary fields have simpler properties, whilst those including descendent fields can be determined with use of local Ward identities. If the correlator in question includes a degenerate field then BPZ equations need to be enforced, which can simplify conformal block computations [18].

In the classic example of 4-point functions, the global Ward identities allow Möbius transformation, mapping 3 of the 4 complex coordinates to $\{0, \zeta, 1, \infty\}$, leaving a single 'cross-ratio' coordinate $\zeta$ for the conformal blocks to be a function of. Explicitly

$$
\left\langle\prod_{i=1}^{4} V_{i}\right\rangle=\sum_{R, \bar{R}} C_{12 R} C_{34 \bar{R}} B_{R}(\zeta) B_{\bar{R}}(\zeta)
$$

for Virasoro operators $V_{i}$. The sum is over the fields' representations in the left and right Virasoro algebras, denoted $R, \bar{R}$; with the sum including structure constants, $C_{i j k}$, and conformal blocks, $B$. These highest-weight representations of the Virasoro algebra are described in terms of Verma modules. They are generated by primary states and are irreducible in the absence of degenerate fields.

Since fields in a correlator can be permuted without change to the result, this translates into allowing different OPEs of the same correlator as different conformal block bases are used for expansion. The equivalence of these OPEs leads to 'crossing symmetry' and introduces additional consistency constraints which allow structure constants and block dimensions to be calculated. This is the conformal bootstrap methodology, and leaves calculation of conformal blocks as the final ingredient for computing correlators [19]. 
Conformal blocks are traditionally computed via Zamolodchikov recursion methods, however in the cases of degenerate fields in the correlators the BPZ equations provide a shortcut to finding them. In special cases these blocks can be expressed simply - for example a 4-point function on the sphere with one degenerate field (with a 2nd order null vector) can be expressed in terms of hypergeometric functions.

The AGT correspondence then makes a connection between the conformal dimensions of the fields in the correlator, and the coordinate $\zeta$, with parameters arising in Nekrasov instanton partition functions, as subsequently described. With the help of this correspondence we then compute the conformal blocks associated to the 6 dessins considered in this study.

The Nekrasov partition function. For generic vev $a$, the general $\mathcal{N}=2$ low energy effective action reads

$$
S_{\text {eff }}=\int \mathrm{d}^{4} x \mathrm{~d}^{4} \theta \mathcal{F}(\Psi)(+ \text { c.c. }),
$$

where $\Psi$ is the $\mathcal{N}=2 \mathrm{~V}$-plet, and the holomorphic function $\mathcal{F}$ is known as the prepotential. First conjectured in [4] and then proven in [20], the prepotential can be solved by

$$
\mathcal{F}=\lim _{\epsilon_{1,2} \rightarrow 0} \epsilon_{1} \epsilon_{2} \log Z_{\mathrm{Nek}}
$$

where $\epsilon_{i}$ 's are known as the deformation parameters, and $Z_{\mathrm{Nek}}$ is the Nekrasov partition function, which reads $Z_{\mathrm{Nek}}=Z_{\text {tree }} Z_{1 \text {-loop }} Z_{\text {inst }}$, where $Z_{\text {tree/1-loop }}$ is the tree/1-loop level partition function and $Z_{\text {inst }}$ denotes the contribution from instantons.

We will now focus on the instanton partition function $Z_{\text {inst }}$. For $\mathrm{SU}(2)$ quiver theories, the Coulomb branches are parametrized by the Coulomb moduli $\vec{a}=\left(a_{1}, a_{2}\right)=(a,-a)$. Each Coulomb modulus is associated with a Young tableau $Y$, in which every box is labelled by a pair $s=(i, j)$ to denote its position. Hence, the instanton partition function depends on $\vec{Y}=\left(Y_{1}, Y_{2}\right)$, the vev $a$, and possibly the mass $m$ of matter in the theory. Let us define $[14]$

$$
E\left(a, Y_{1}, Y_{2}, s\right) \equiv a-\epsilon_{1} L_{Y_{2}}(s)+\epsilon_{2}\left(A_{Y_{1}}(s)+1\right)
$$

with

$$
L_{Y_{2}}(s)=k_{i}-j, A_{Y_{1}}(s)=k_{j}^{\prime}-i,
$$

where $k_{i}$ is the length of $i^{\text {th }}$ row of $Y_{2}$, and $k_{j}^{\prime}$ is the height of $j^{\text {th }}$ column of $Y_{2}$. Let $I, J$ label the gauge nodes. Then

$$
\begin{aligned}
& z_{\mathrm{bifund}}\left(a^{I}, \vec{Y}^{I} ; a^{J}, \vec{Y}^{J} ; m\right) \\
& \quad \equiv \prod_{i, j=1}^{2}\left(\prod_{s \in Y_{i}^{I}}\left(E\left(a_{i}^{I}-a_{j}^{J}, Y_{i}^{I}, Y_{j}^{J}, s\right)-m\right) \prod_{s \in Y_{j}^{J}}\left(\epsilon-E\left(a_{j}^{J}-a_{i}^{I}, Y_{j}^{J}, Y_{i}^{I}, s\right)-m\right)\right),
\end{aligned}
$$

where $\epsilon=\epsilon_{1}+\epsilon_{2}$. For (anti-)fundamentals,

$$
z_{\text {fund }}(\vec{a}, \vec{Y}, m) \equiv \prod_{i=1}^{2} \prod_{s \in Y_{i}}\left(\phi\left(a_{i}, s\right)-m+\epsilon\right), z_{\text {antifund }}(\vec{a}, \vec{Y}, m) \equiv z_{\text {fund }}(\vec{a}, \vec{Y}, \epsilon-m),
$$

where $\phi\left(a_{i}, s\right)=a_{i}+\epsilon_{1}(i-1)+\epsilon_{2}(j-1)$. For adjoint chiral and vector multiplets,

$$
z_{\text {adj }}(\vec{a}, \vec{Y}, m) \equiv z_{\text {bifund }}(\vec{a}, \vec{Y} ; \vec{a}, \vec{Y} ; m), z_{\mathrm{vec}}(\vec{a}, \vec{Y}) \equiv \frac{1}{z_{\text {adj }}(\vec{a}, \vec{Y}, 0)} .
$$


The AGT correspondence. The (chiral) VO can be written as $V_{\alpha}=: \mathrm{e}^{2 \alpha \phi}$ : for some free scalar $\phi$. If we introduce some background charge $Q$, by considering the OPE between stress tensor and the $\mathrm{VO}$, we get the conformal dimension of $V_{\alpha}$, which reads $\Delta_{\alpha}=\alpha(Q-\alpha)$. Likewise, the OPE between stress tensors yields the central charge $c=1+6 Q^{2}$.

Now we are ready to bridge the CBs and instanton partition functions. Originally, this was done for Liouville theory in [1]. We can fix the scale by setting $b=\frac{\epsilon_{1}}{\sqrt{\epsilon_{1} \epsilon_{2}}}$, where $Q=b+$ $\frac{1}{b}$ and $b$ is the parameter coming from the Liouville potential. Therefore, $Q=\frac{\epsilon_{1}+\epsilon_{2}}{\sqrt{\epsilon_{1} \epsilon_{2}}} \equiv \frac{\epsilon}{\sqrt{\epsilon_{1} \epsilon_{2}}}$. Consider a quiver consisting of an $\mathrm{SU}(2)$ gauge group with $2 \mathrm{SU}(2)$ antifundamentals and $2 \mathrm{SU}(2)$ fundamentals with mass parameters $\mu_{1,2}$ and $\mu_{3,4}$ respectively. Then the instanton partition function reads

$$
Z_{\text {inst }}=\sum_{Y_{1,2}} \frac{\mathrm{e}^{2 \pi \mathrm{i} \tau\left(\left|Y_{1}\right|+\left|Y_{2}\right|\right)}}{\left(1-\mathrm{e}^{2 \pi \mathrm{i} \tau}\right)^{\frac{1}{2}\left(\mu_{1}+\mu_{2}\right)\left(2 \epsilon-\left(\mu_{3}+\mu_{4}\right)\right)}} z_{\mathrm{vec}}(\vec{a}, \vec{Y}) z_{\text {matter }},
$$

where the denominator correpsonds to the decoupling of a U(1) factor, and

$$
z_{\text {matter }}=z_{\text {antifund }}\left(\vec{a}, \vec{Y}, \mu_{1}\right) z_{\text {antifund }}\left(\vec{a}, \vec{Y}, \mu_{2}\right) z_{\text {fund }}\left(\vec{a}, \vec{Y}, \mu_{3}\right) z_{\text {fund }}\left(\vec{a}, \vec{Y}, \mu_{4}\right) .
$$

The instanton number $\left|Y_{i}\right|$ is the number of boxes in $Y_{i}$. Then under the following AGT dictionary,

$$
\begin{aligned}
\frac{\mu_{1}}{\sqrt{\epsilon_{1} \epsilon_{2}}}=\alpha_{2}+\alpha_{1}-\frac{Q}{2}, & \frac{\mu_{2}}{\sqrt{\epsilon_{1} \epsilon_{2}}}=\alpha_{2}-\alpha_{1}+\frac{Q}{2}, & \frac{\mu_{3}}{\sqrt{\epsilon_{1} \epsilon_{2}}}=\alpha_{3}+\alpha_{4}-\frac{Q}{2}, \\
\frac{\mu_{4}}{\sqrt{\epsilon_{1} \epsilon_{2}}}=\alpha_{3}-\alpha_{4}+\frac{Q}{2}, & \frac{a}{\sqrt{\epsilon_{1} \epsilon_{2}}}=\alpha_{\text {int }}-\frac{Q}{2}, & \mathrm{e}^{2 \pi \mathrm{i} \tau}=\zeta,
\end{aligned}
$$

the instanton partition function is equal to $\mathcal{B}_{\alpha_{\text {int }}}\left(\alpha_{i} \mid \zeta\right)$, where the conformal block from $\left\langle V_{\alpha_{1}} V_{\alpha_{2}} V_{\alpha_{3}} V_{\alpha_{4}}\right\rangle$ as in (2.1) can be written as $B=\zeta^{\Delta_{\alpha_{\text {int }}}-\Delta_{\alpha_{1}}-\Delta_{\alpha_{2}}} \mathcal{B}_{\alpha_{\text {int }}}\left(\alpha_{i} \mid \zeta\right)$ and "int" stands for (the VO in) the intermediate channel. One may check this perturbatively, and at level $|Y|_{\max }, \mathcal{B}_{\alpha_{\text {int }}}$ and $Z_{\text {inst }}$ should agree up to $\mathcal{O}\left(\zeta^{|Y|_{\max }+1}\right)[1,21]$. Notice that when we have $c=1 \mathrm{CBs}$, viz, $Q=0$, the AGT relation is simplified to

$$
\frac{\mu_{1}}{\sqrt{\epsilon_{1} \epsilon_{2}}}=\alpha_{1}+\alpha_{2}, \frac{\mu_{2}}{\sqrt{\epsilon_{1} \epsilon_{2}}}=\alpha_{1}-\alpha_{2}, \frac{\mu_{3}}{\sqrt{\epsilon_{1} \epsilon_{2}}}=\alpha_{3}+\alpha_{4}, \frac{\mu_{4}}{\sqrt{\epsilon_{1} \epsilon_{2}}}=\alpha_{3}-\alpha_{4}, \frac{a}{\sqrt{\epsilon_{1} \epsilon_{2}}}=\alpha_{\mathrm{int}} .
$$

It is also possible to build a similar correspondence between gauge theories and minimal models. In [15], it was shown that $Z_{\text {inst }}$ should recover the CBs for minimal models if we put further restrictions to the Young tableaux pairs known as the Burge condition. For a minimal model, we may write the central charge as

$$
c=1-6 \frac{\left(p^{\prime}-p\right)^{2}}{p^{\prime} p},
$$

where $p^{\prime}, p$ are coprime integers and $p^{\prime}>p>1$. The spectrum is finite and all the VOs have conformal dimensions

$$
\Delta_{r, s}=\frac{\left(p^{\prime} r-p s\right)^{2}-\left(p^{\prime}-p\right)^{2}}{4 p^{\prime} p}
$$


for integers $r, s$ and $1 \leq r<p, 1 \leq s<p^{\prime}$. In other words, they should all live in the Kac table. Then the instanton partition function leads to well-defined A-series minimal models under (2.11) if the partitions $Y_{1,2}$ are restricted to be Burge pairs, that is, they satisfy [15]

$$
Y_{2, R}-Y_{1, R+s-1} \geq 1-r, Y_{1, R}-Y_{2, R+p-s-1} \geq 1-p^{\prime}+r
$$

where $Y_{i, R}$ denotes (the number of boxes in) the $R^{\text {th }}$ row of $Y_{i}$. In particular, the deformation parameters can now be written in terms of the screening charges as

$$
\frac{\epsilon_{1}}{\sqrt{\epsilon_{1} \epsilon_{2}}}=-\mathrm{i} \sqrt{\frac{p}{p^{\prime}}}, \frac{\epsilon_{2}}{\sqrt{\epsilon_{1} \epsilon_{2}}}=\mathrm{i} \sqrt{\frac{p^{\prime}}{p}} .
$$

\subsection{From 4d to 5d instanton partition functions and A-model topological string partition functions}

For type II string/M-theory (whose brane configurations are discussed in appendix B) compactified on a Calabi-Yau 3-fold, the amplitudes at genus $g$ correspond to the A-model string amplitudes of the $\mathrm{CY}_{3}$ which enumerates the holomorphic functions from genus $g$ Riemann surfaces to the $\mathrm{CY}_{3}[22,23]$. The topological amplitudes for toric CY threefolds can be computed by topological vertices introduced in [24-26]. A topological vertex is a trivalent vertex as the (black) dual graph of the (grey) toric diagram:

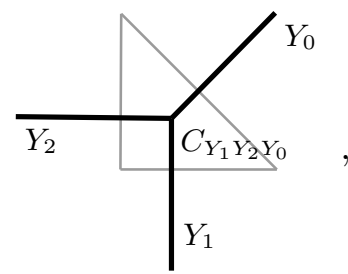

where $Y_{i}$ 's are the Young tableaux associated to the legs, and $C_{Y_{1} Y_{2} Y_{0}}(q)$ is the factor associated to the vertex, which can be expressed in terms of Schur and skew-Schur functions [24]. Albeit not labelled explicitly, each leg also has a direction such that the three legs attached to the same vertex all have outcoming or incoming directions. Then each leg is assigned a vector $\boldsymbol{v}_{i}=\left(v_{i 1}, v_{i 2}\right)$ in that direction, such that the sum of the three vectors vanishes due to charge conservation and $\operatorname{det}\left(\boldsymbol{v}_{i}, \boldsymbol{v}_{i+1}\right)= \pm 1\left(i \in \mathbb{Z}_{3}\right)$. Now two topological vertices

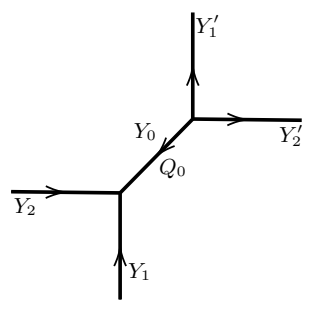

can be glued as

$$
\sum_{Y_{0}} C_{Y_{1}^{\mathrm{T}} Y_{2}^{\mathrm{T}} Y_{0}^{\mathrm{T}}}(q)(-1)^{(n+1)\left|Y_{0}\right|} q^{-n \kappa\left(Y_{0}\right) / 2} Q_{0}^{\left|Y_{0}\right|} C_{Y_{1}^{\prime} Y_{2}^{\prime} Y_{0}}
$$


where $\kappa$ is related to quadratic Casimir of the representation corresponding to $\left|Y_{0}\right|$, namely, $\kappa\left(Y_{0}\right)=\sum_{i} y_{i}\left(y_{i}-2 i+1\right)$ with $y_{i}$ being the number of boxes in the $i^{\text {th }}$ row. The framing number $n$ equals $\operatorname{det}\left(\boldsymbol{v}_{\text {in }}, \boldsymbol{v}_{\text {out }}\right)$, where the two vectors are chosen such that $\boldsymbol{v}_{\text {in }} \cdot \boldsymbol{v}_{\text {out }}>0$. The parameter $Q_{0}$ is the (exponentiated) Kähler parameter for the 2-cycle corresponding to the line in the dual toric diagram.

In [25], the above is extended to a refined topological vertex as

$$
\begin{aligned}
C_{Y_{1} Y_{2} Y_{0}}(q, t)= & \left(\frac{q}{t}\right)^{\left(\left\|Y_{2} \mid\right\|^{2}+\left\|Y_{0}\right\|^{2}\right) / 2} t^{\kappa\left(Y_{2}\right) / 2} P_{Y_{0}^{\mathrm{T}}}\left(t^{-\rho} ; q, t\right) \\
& \times \sum_{\eta}\left(\frac{q}{t}\right)^{\left(|\eta|+\left|Y_{1}\right|-\left|Y_{2}\right|\right) / 2} s_{Y_{1}^{\mathrm{T}} / \eta}\left(t^{-\rho} q^{-Y_{0}}\right) s_{Y_{2} / \eta}\left(t^{-Y_{0}^{\mathrm{T}}} q^{-\rho}\right),
\end{aligned}
$$

where $P_{Y_{0}^{\mathrm{T}}}\left(t^{-\rho} ; q, t\right)$ is the Macdonald function and $s_{\alpha / \beta}$ 's are the skew-Schur functions. The squared double slash denotes the quadratic sum of the number of boxes in each row of the Young tableau. Notice that the three Young tableaux are not cyclically symmetric and $Y_{0}$ corresponds to the preferred leg for gluing. One may check that when the $\Omega$-background parameters satisfy $q=t$, we would recover the unrefined topological vertex.

Define the framing factors,

$$
f_{Y}(q, t)=(-1)^{|Y|} q^{|| Y^{\mathrm{T}} \|^{2} / 2} t^{-\|Y\|^{2} / 2}, \quad \tilde{f}_{Y}(q, t)=(-1)^{|Y|} q^{\left(\| Y^{\mathrm{T}}||^{2}+|Y|\right) / 2} t^{-\left(\|Y\|^{2}+|Y|\right) / 2},
$$

and the edge factor, $\left(-Q_{0}\right)^{\left|Y_{0}\right|} \times$ [framing factor]. Then the topological string partition function takes the sum over all the Young tableaux of internal legs ${ }^{1}$ as

$$
Z_{\text {topo }}=\sum_{Y_{i}} \prod_{\text {edges }}[\text { edge factor }] \prod_{\text {vertices }}[\text { vertex factor }]
$$

Again, let us contemplate the SU(2) gauge theory with 4 flavours. The dual web diagram is

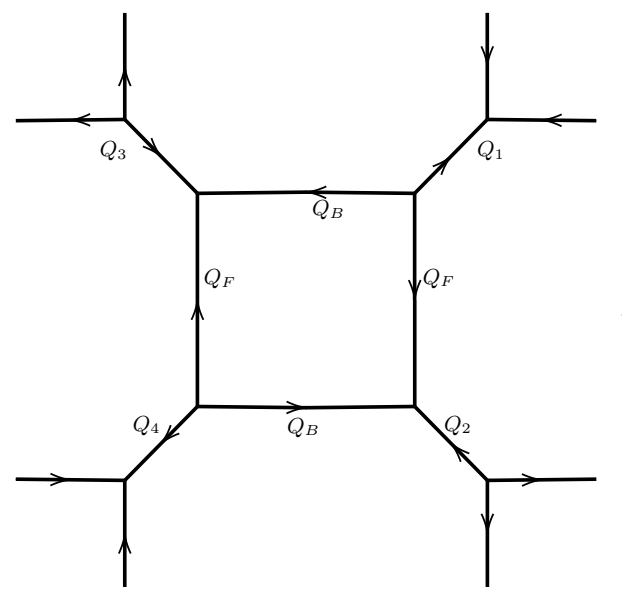

\footnotetext{
${ }^{1}$ The Young tableaux of external legs would be $\emptyset$.
} 
Following the gluing process, the partition function reads ${ }^{2}$

$$
\begin{aligned}
Z_{\text {topo }}= & \sum_{\lambda \rho \nu}\left(-Q_{F}\right)^{\left|\lambda_{1}\right|} \tilde{f}_{\lambda_{1}}(q, t)\left(-Q_{F}\right)^{\left|\lambda_{2}\right|} \tilde{f}_{\lambda_{2}}(t, q)\left(-Q_{B}\right)^{\left|\rho_{1}\right|} f_{\rho_{1}^{\mathrm{T}}}(q, t)\left(-Q_{B}\right)^{\left|\rho_{2}\right|} f_{\rho_{2}^{\mathrm{T}}}(t, q) \\
& \times\left(-Q_{1}\right)^{\left|\nu_{1}\right|}\left(-Q_{2}\right)^{\left|\nu_{2}\right|}\left(-Q_{3}\right)^{\left|\nu_{3}\right|}\left(-Q_{4}\right)^{\left|\nu_{4}\right|} C_{\lambda_{1}^{\mathrm{T}} \nu_{1}^{\mathrm{T}} \rho_{1}^{\mathrm{T}}}(q, t) C_{\nu_{4} \lambda_{1} \rho_{2}}(q, t) \\
& \times C_{\lambda_{2}^{\mathrm{T}} \nu_{2}^{\mathrm{T}} \rho_{2}^{\mathrm{T}}}(t, q) C_{\nu_{3} \lambda_{2} \rho_{1}}(t, q) C_{\nu_{3}^{\mathrm{T}} \emptyset \emptyset}(q, t) C_{\emptyset \nu_{2} \emptyset}(q, t) C_{\nu_{4}^{\mathrm{T}} \emptyset \emptyset}(t, q) C_{\emptyset \nu_{1} \emptyset}(t, q) .
\end{aligned}
$$

Recall the $4 \mathrm{~d}$ instanton partition function (2.9), which can be lifted to $5 \mathrm{~d}$ as [28]

$$
Z_{\text {inst }, 5 \mathrm{~d}}=\frac{1}{Z_{\mathrm{U}(1), 5 \mathrm{~d}}} \sum_{Y_{1,2}} \mathrm{e}^{2 \pi \mathrm{i} \tau\left(\left|Y_{1}\right|+\left|Y_{2}\right|\right)} z_{\mathrm{vec}, 5 \mathrm{~d}}(\vec{a}, \vec{Y}) z_{\text {matter }, 5 \mathrm{~d}} .
$$

It is discussed in [28-32] that under the parameter identification ${ }^{3}$

$$
q=\mathrm{e}^{-R \epsilon_{1}}, t=\mathrm{e}^{R \epsilon_{2}}, Q_{i}=\mathrm{e}^{-R\left(\mu_{i}-a\right)}, Q_{B}=\mathrm{e}^{2 \pi \mathrm{i} \tau} \mathrm{e}^{\frac{1}{2} R\left(-4 a+\sum_{i} \mu_{i}\right)}, Q_{F}=\mathrm{e}^{-2 R a},
$$

where $R$ is the radius of the compactified dimension $S^{1}, Z_{\text {topo }}$ reproduces $Z_{\text {inst, } 5 \mathrm{~d}}$ up to perturbative part and $\mathrm{U}(1) /$ extra factor $[30,33-38]$. Notice that when $\epsilon_{1}=-\epsilon_{2}$, i.e., $Q=0$, we have the unrefinement $q=t$. In particular, under the $4 \mathrm{~d}$ limit $R \rightarrow 0$, the $4 \mathrm{~d}$ topological A-model partition function would give the $4 \mathrm{~d}$ instanton partition function.

\subsection{From topological string partition functions to Seiberg-Witten curves}

As aforementioned, the low energy effective theory for $4 \mathrm{~d} \mathcal{N}=2$ can be encoded by the prepotential $\mathcal{F}$, where $\mathcal{F}=\lim _{\epsilon_{1,2} \rightarrow 0} \epsilon_{1} \epsilon_{2} \log Z_{\text {Nek }}$ in terms of the Nekrasov partition function ${ }^{4}$ which is in turn naturally related to topological partition functions in the A-model.

On the other hand, in the SW solution, the prepotential can be determined by the SW curve. Given such auxiliary curve $\Sigma$, it is possible to translate into the form $\lambda^{2}=q(z)$, where $\lambda$ is the Seiberg-Witten differential, and $q(z)$ is the meromorphic quadratic differential on the Gaiotto curve $\mathcal{C}[5,39]$. In this subsection, we demonstrate how this translation runs for the theory with a single $\mathrm{SU}(2)$ factor and $N_{f}=4$. This theory will constitute our running example throughout the paper.

To begin, following $\S 9.1$ of [39], $\Sigma$ for the $\mathrm{SU}(2)$ with $N_{f}=4$ theory in hyperelliptic form is

$$
\frac{f}{\tilde{z}}\left(\tilde{x}-\tilde{\mu}_{1}\right)\left(\tilde{x}-\tilde{\mu}_{2}\right)+\left(f^{\prime} \tilde{z}\right)\left(\tilde{x}-\tilde{\mu}_{3}\right)\left(\tilde{x}-\tilde{\mu}_{4}\right)=\tilde{x}^{2}-u
$$

where $f$ and $f^{\prime}$ are complex numbers and $u$ parametrizes the space of supersymmetric vacua, viz, the $u$-plane. We first choose the coordinate of $\tilde{z}$ so that $f^{\prime}=1$. Completing a square in $\tilde{x}$ by defining

$$
x=\tilde{x}+\frac{\frac{f}{\tilde{z}}\left(\tilde{\mu}_{1}+\tilde{\mu}_{2}\right)+\tilde{z}\left(\tilde{\mu}_{3}+\tilde{\mu}_{4}\right)}{2\left(1-\tilde{z}-\frac{f}{\tilde{z}}\right)},
$$

\footnotetext{
${ }^{2}$ More generally, for $\mathrm{SU}\left(N_{c}\right)$ gauge group with $N_{f}=2 N_{c}$, the partition function was given in [27].

${ }^{3}$ Often $Q_{i}$ would be written as $\mathrm{e}^{-R\left(-\mu_{i}-a\right)}$ for $i=2,4$. However, due to invariance under Weyl group symmetry, the Nekrasov partition function should not change under $\mu_{2,4} \rightarrow-\mu_{2,4}$.

${ }^{4}$ The finite non-zero deformation parameters could also physically make sense for SW curves, for example in the context of topological B-models as in appendix A.
} 

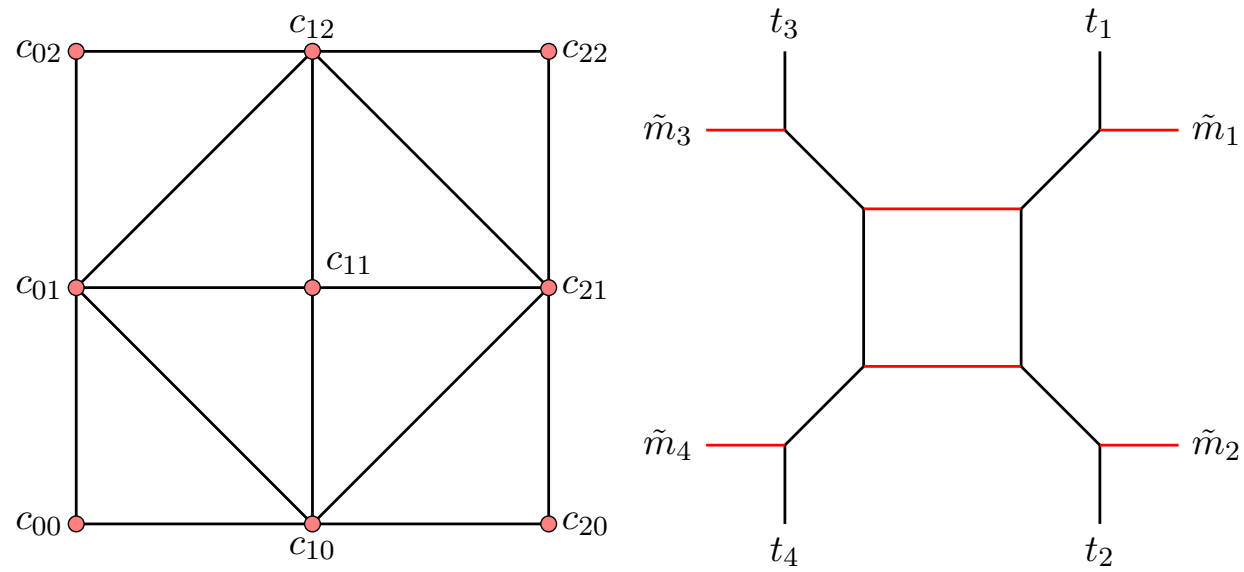

Figure 1. The toric diagram and its dual diagram for SU(2) with 4 flavours.

we obtain $x^{2}=g(\tilde{z})$ where $g$ has double poles at $c_{1,2}(f)$. Now rescale $z=\tilde{z} / c_{1}(f)$ so that the poles are at $z=1, \zeta$, and we get

$$
x^{2}=\frac{P(z)}{(z-1)^{2}(z-\zeta)^{2}}
$$

for some quartic polynomial $P(z)$ determined by $\tilde{\mu}_{i}, \zeta$ and $u$. Then $a$ and its magnetic dual $a_{D}$ can be obtained by integrating the SW differential $\lambda \equiv x \mathrm{~d} z / z$ along $A$ - and $B$-cycles:

$$
a=\oint_{A} \lambda, a_{D}=\frac{\partial \mathcal{F}}{\partial a}=\oint_{B} \lambda \text {. }
$$

Construction of SW curves from toric diagrams. For 5 d gauge theories, the 5-brane web diagrams can be used to construct SW curves. In fact, such a web diagram is exactly the same as the dual toric diagram in the geometric engineering in section 2.2 [40, 41]. The standard algorithm of constructing SW curves from toric diagrams are proposed in [42] and elaborated in [43]. Here, we will still focus on $\mathrm{SU}(2)$ with 4 flavours, where the web/dual diagram is reproduced in figure 1 , along with its toric diagram.

For each vertex $(i, j)$ in the toric diagram, we assign a non-zero number $c_{i j}$. With these coefficients, the SW curve is given as

$$
\sum_{i, j} c_{i j} t^{i} w^{j}=0
$$

By multiplying an overall constant to this equation, we can impose

$$
c_{22}=1
$$

without the loss of generality. There are four boundaries, so the boundary conditions according to the toric diagram now are

$$
\begin{aligned}
& |w| \gg 1, \quad c_{02} w^{2}+c_{12} t w^{2}+c_{22} t^{2} w^{2}=c_{22} w^{2}\left(t-t_{1}\right)\left(t-t_{3}\right), \\
& \left|w^{-1}\right| \gg 1, \quad c_{20} t^{2}+c_{10} t+c_{00}=c_{20}\left(t-t_{2}\right)\left(t-t_{4}\right), \\
& |t| \gg 1, \quad c_{20} t^{2}+c_{21} t^{2} w+c_{22} t^{2} w^{2}=c_{22} t^{2}\left(w-\tilde{m}_{1}\right)\left(w-\tilde{m}_{2}\right), \\
& \left|t^{-1}\right| \gg 1, \quad c_{02} w^{2}+c_{01} w+c_{00}=c_{02}\left(w-\tilde{m}_{3}\right)\left(w-\tilde{m}_{4}\right),
\end{aligned}
$$


where $t$ and $w$ can be thought of as the horizontal and vertical coordinates of the diagram respectively. In order for the curve to satisfy all the conditions consistently, we need the compatibility condition which reads

$$
\tilde{m}_{1} t_{1}^{-1} \tilde{m}_{2} t_{2}=\tilde{m}_{3} t_{3} \tilde{m}_{4} t_{4}^{-1} .
$$

Since the SW differential is invariant under the rescaling of $t$, we can impose

$$
t_{2}=1
$$

for simplicity. Also, by rescaling $w$, we can further impose

$$
\tilde{m}_{1} t_{1}^{-1} \tilde{m}_{2} t_{2}=1 .
$$

This condition turns out to correspond to the traceless condition of the vacuum expectation value of the $\mathrm{SU}(2)$ vector multiplet [29]. The instanton factor is the geometric average of $t_{i}$, which is

$$
\zeta \equiv\left(\frac{t_{3} t_{4}}{t_{1} t_{2}}\right)^{\frac{1}{2}}
$$

The only undetermined coefficient $c_{11}=-U^{\prime}$ is interpreted as Coulomb moduli parameter. Defining a parameter $S \equiv \tilde{m}_{1} \tilde{m}_{2} \tilde{m}_{3} \tilde{m}_{4}$, we have

$$
t_{1}=\tilde{m}_{1} \tilde{m}_{2}, \quad t_{2}=1, \quad t_{3}=\tilde{m}_{1} \tilde{m}_{2}(S)^{-\frac{1}{2}} \zeta, \quad t_{4}=(S)^{\frac{1}{2}} \zeta,
$$

and thus, the $\mathrm{SW}$ curve for $5 \mathrm{~d} \mathcal{N}=1 \mathrm{SU}(2)$ gauge theory with $N_{f}=4$ is

$$
\begin{array}{r}
\mathrm{t}^{2}\left(w-\tilde{m}_{1}\right)\left(w-\tilde{m}_{2}\right)-\mathrm{t}\left(w^{2} \tilde{m}_{1} \tilde{m}_{2}\left(1+\zeta(S)^{-\frac{1}{2}}\right)+w U^{\prime}+\tilde{m}_{1} \tilde{m}_{2}\left(1+\zeta(S)^{\frac{1}{2}}\right)\right) \\
+\zeta(S)^{-\frac{1}{2}}\left(\tilde{m}_{1} \tilde{m}_{2}\right)^{2}\left(w-\tilde{m}_{3}\right)\left(w-\tilde{m}_{4}\right)=0 .
\end{array}
$$

The 4d limit curve. Till now, the SW curve is a $5 \mathrm{~d}$ curve, its $4 \mathrm{~d} \mathrm{SW}$ curve can be obtained by taking the vanishing limit of size of compactification circle $\beta \rightarrow 0$, where we have

$$
w=e^{-\beta v}, \tilde{m}_{i}=e^{-\beta \mu_{i}} .
$$

We then expand the $5 \mathrm{~d}$ Coulomb parameter $U^{\prime}$ as

$$
U^{\prime}=\sum_{k=0}^{\infty} u_{k} \beta^{k}
$$

In particular,

$$
u_{0}=-2(1+\zeta), u_{1}=2(\zeta+1)\left(\mu_{1}+\mu_{2}\right) .
$$

For the $N_{f}=4$ curve, we then have the $4 \mathrm{~d}$ limit

$$
t^{2}\left(v-\mu_{1}\right)\left(v-\mu_{2}\right)+\zeta\left(v-\mu_{3}\right)\left(v-\mu_{4}\right)+t(1+\zeta)\left(-v^{2}+\frac{\zeta v}{1+\zeta} \sum_{i=1}^{4} \mu_{i}+U\right)=0,
$$

where

$$
U=-\frac{u_{2}}{1+\zeta}-\left(\mu_{1}+\mu_{2}\right)^{2}-\frac{\zeta}{4(1+\zeta)}\left(\sum_{i=1}^{4} \mu_{i}\right)^{2}
$$


Reparametrization. Let us rewrite the curve as

$$
\frac{t}{1+\zeta}\left(v-\mu_{1}\right)\left(v-\mu_{2}\right)-v^{2}+\frac{\zeta v \sum_{i=1}^{4} \mu_{i}}{1+\zeta}+U+\frac{\zeta}{t} \frac{1}{1+\zeta}\left(v-\mu_{3}\right)\left(v-\mu_{4}\right)=0 .
$$

Redefining

$$
\begin{aligned}
f / \tilde{z} & =\frac{t}{1+\zeta}, & f^{\prime} \tilde{z}=\frac{\zeta}{t(1+\zeta)} \\
\tilde{x} & =v-\frac{\zeta}{2(1+\zeta)} \sum_{i=1}^{4} \mu_{i}, & \tilde{\mu}_{i}=\mu_{i}-\frac{\zeta}{2(1+\zeta)} \sum_{i=1}^{4} \mu_{i}, \\
u & =\left(\frac{\zeta}{2(1+\zeta)} \sum_{i=1}^{4} \mu_{i}\right)^{2}+U &
\end{aligned}
$$

recovers the curve of form (2.27), which is reproduced here:

$$
\frac{f}{\tilde{z}}\left(\tilde{x}-\tilde{\mu}_{1}\right)\left(\tilde{x}-\tilde{\mu}_{2}\right)+\left(f^{\prime} \tilde{z}\right)\left(\tilde{x}-\tilde{\mu}_{3}\right)\left(\tilde{x}-\tilde{\mu}_{4}\right)=\tilde{x}^{2}-u .
$$

\subsection{From Seiberg-Witten curves to dessins d'enfants}

We begin with a refresher on some preliminary definitions and key results [44, 45].

Definition 2.1. A dessin d'enfant, or child's drawing, is an ordered pair $(X, \mathcal{D})$, where $X$ is an oriented compact topological surface and $\mathcal{D} \subset X$ is a finite graph, such that

1. $\mathcal{D}$ is a connected bipartite graph, and

2. $X \backslash \mathcal{D}$ is the union of finitely many topological discs that are the faces of $\mathcal{D}$.

There is a bijection between the dessins and Belyi maps known as the Grothendieck correspondence [46], where

Definition 2.2. A Belyi map $\beta$ is a holomorphic map from the Riemann surface $X$ to $\mathbb{P}^{1}$ ramified at only 3 points, which can be taken to be $\{0,1, \infty\} \in \mathbb{P}^{1}$.

Recall that ramification means that the only points $\tilde{x} \in X$ where $\left.\frac{d}{d x} \beta(x)\right|_{\tilde{x}}=0$ are such that $\beta(\tilde{x})=0,1$ or $\infty$. In other words, the local Taylor expansion of $\beta(x)$ about the pre-images $\tilde{x}$ of $\{0,1, \infty\}$ have (at least) vanishing linear term.

From Belyi maps to dessins. We can associate $\beta(x)$ to a dessin via its ramification indices: the order of vanishing of the Taylor series for $\beta(x)$ at $\tilde{x}$ is the ramification index $r_{\beta(\tilde{x}) \in\{0,1, \infty\}}(i)$ at that $i^{\text {th }}$ point. By convention, we mark one white node for the $i^{\text {th }}$ preimage of 0 with $r_{0}(i)$ edges emanating therefrom. Similarly, we mark one black node for the $j^{\text {th }}$ pre-image of 1 with $r_{1}(j)$ edges. We then connect the nodes with the edges, joining only black with white, such that each face is a polygon with $2 r_{\infty}(k)$ sides. In other words, there is one pre-image of $\infty$ corresponding to each polygon of $\mathcal{D}$. Moreover, there is a cyclic ordering arising from local monodromy winding around vertices, i.e., around local covering sheets that contain a common point.

The power of dessins comes from Belyi's remarkable theorem. 
Theorem 2.1. There exists an algebraic model of $X$ (as a Riemann surface) defined over $\overline{\mathbb{Q}}$ iff there exists a Belyi map on $X$.

Thus, the existence of a dessin on $X$ is equivalent to $X$ admitting an algebraic equation over the algebraic numbers. Moreover, the Galois group $\operatorname{Gal}(\overline{\mathbb{Q}}: \mathbb{Q})$ acts faithfully on the space of dessins.

Quadratic differentials. A (holomorphic) quadratic differential $q$ on a Riemann surface $X$ is a holomorphic section of the symmetric square of the contangent bundle. In terms of local coordinates $z, q=f(z) \mathrm{d} z \otimes \mathrm{d} z$, for some holomorphic function $f(z)$.

A curve $\gamma(t) \subset X$ can be classified by $q$ as

- Horizontal trajectory: $f(\gamma(t)) \dot{\gamma}(t)^{2}>0$;

- Vertical trajectory: $f(\gamma(t)) \dot{\gamma}(t)^{2}<0$.

Locally, one can find coordinates so that horizontal tracjectories look like concentric circles while vertical trajectories look like rays emanating from a single point.

Then we can define the Strebel differential:

Definition 2.3. For a Riemann surface $X$ of genus $g \geq 0$ with $n \geq 1$ marked points $\left\{p_{1}, \ldots, p_{n}\right\}$ such that $2-2 g<n$, and a given $n$-tuple $a_{i=1, \ldots, n} \in \mathbb{R}^{+}$, a Strebel differential $q=f(z) \mathrm{d} z^{2}$ is a quadratic differential such that

- $f$ is holomorphic on $X \backslash\left\{p_{1}, \ldots, p_{n}\right\}$;

- $f$ has a second-order pole at each $p_{i}$;

- the union of all non-compact horizontal trajectories of $q$ is a closed subset of $X$ of measure 0;

- every compact horizontal of $q$ is a simple loop $A_{i}$ centered at $p_{i}$ such that $a_{i}=$ $\oint_{A_{i}} \sqrt{q}$. (Here the branch of the square root is chosen so that the integral has a positive value with respect to the positive orientation of $A_{i}$ that is determined by the complex structure of $X$.)

The upshot is that [47]

Theorem 2.2. The Strebel differential is the pull-back, by a Belyi map $\beta: X \rightarrow \mathbb{P}^{1}$, of a quadratic differential on $\mathbb{P}^{1}$ with 3 punctures,

$$
q=\beta^{*}\left(\frac{\mathrm{d} \zeta^{2}}{4 \pi^{2} \zeta(1-\zeta)}\right)=\frac{(\mathrm{d} \beta)^{2}}{4 \pi^{2} \beta(1-\beta)}=\frac{\left(\beta^{\prime}\right)^{2}}{4 \pi^{2} \beta(1-\beta)} \mathrm{d} z^{2},
$$

where $z$ and $\zeta$ are coordinates on $X$ and $\mathbb{P}^{1}$ respectively.

Recall the definition of the SW differential

$$
\lambda=v \frac{\mathrm{d} z}{z} .
$$




\begin{tabular}{|c|c|c|c|}
\hline Graph & $\beta(t)$ & Ramification & Strebel $q$ \\
\hline$\Gamma(3)$ & $\frac{t^{3}(t+6)^{3}\left(t^{2}-6 t+36\right)^{3}}{1728(t-3)^{3}\left(t^{2}+3 t+9\right)^{3}}$ & {$\left[3^{4}\left|2^{6}\right| 3^{4}\right]$} & $-\frac{9 t\left(t^{3}+216\right)}{4 \pi^{2}\left(t^{3}-27\right)^{2}}$ \\
\hline$\Gamma_{0}(4) \cap \Gamma(2)$ & $\frac{\left(t^{4}+224 t^{2}+256\right)^{3}}{1728 t^{2}(t-4)^{4}(t+4)^{4}}$ & {$\left[3^{4}\left|2^{6}\right| 4^{2}, 2^{2}\right]$} & $-\frac{4 t^{4}+896 t^{2}+1024}{4 \pi^{2} t^{2}\left(t^{2}-16\right)^{2}}$ \\
\hline$\Gamma_{1}(5)$ & $\frac{\left(t^{4}+248 t^{3}+4064 t^{2}+22312 t+40336\right)^{3}}{1728(t+5)\left(t^{3}-t-31\right)^{5}}$ & {$\left[3^{4}\left|2^{6}\right| 5^{2}, 1^{2}\right]$} & $-\frac{t^{4}+248 t^{3}+4064 t^{2}+22312 t+40336}{4 \pi^{2}(t+5)^{2}\left(t^{2}-t-31\right)^{2}}$ \\
\hline$\Gamma_{0}(6)$ & $\frac{(t+7)^{3}\left(t^{3}+237 t^{2}+1443 t+2287\right)^{3}}{1728(t+3)^{2}(t+4)^{3}(t-5)^{6}}$ & {$\left[3^{4}\left|2^{6}\right| 6,3,2,1\right]$} & $-\frac{(t+7)\left(t^{3}+237 t^{2}+1443 t+2287\right)}{4 \pi^{2}(t+5)^{2}(t+3)^{2}(t+4)^{2}}$ \\
\hline$\Gamma_{0}(8)$ & $\frac{\left(t^{4}+240 t^{3}+2144 t^{2}+3840 t+256\right)^{3}}{1728 t(t+4)^{2}(t-4)^{8}}$ & {$\left[3^{4}\left|2^{6}\right| 8,2,1^{2}\right]$} & $-\frac{t^{4}+240 t^{3}+2144 t^{2}+3840 t+256}{4 \pi^{2} t^{2}\left(t^{2}-16\right)^{2}}$ \\
\hline$\Gamma_{0}(9)$ & $\frac{(t+6)^{3}\left(t^{3}+234 t^{2}+756 t+2160\right)^{3}}{1728\left(t^{2}+3 t+9\right)(t-3)^{9}}$ & {$\left[3^{4}\left|2^{6}\right| 9,1^{3}\right]$} & $-\frac{(t+6)\left(t^{3}+234 t^{2}+756 t+2160\right)}{4 \pi^{2}\left(t^{3}-27\right)^{2}}$ \\
\hline
\end{tabular}

Table 1. The list of the six genus-zero, torsion-free, congruence subgroups of the modular group $\Gamma$, of index 12. The corresponding Belyi maps $\beta(t)$ and their ramification indices, as well as the Strebel differentials are also shown. Note that the ramification indices for all 6 are such that there are 4 pre-images of 0 of order 3 and 6 pre-images of 1 of order 2 . The pre-images of $\infty$ (aka the cusp widths) all add to 12, as do the ramification indices for 0 and 1 . This is required by the fact that all the subgroups are of index 12 within $\Gamma$.

Then

$$
q=\lambda^{2}=v^{2} \frac{\mathrm{d} z^{2}}{z^{2}}=: \phi(z) \mathrm{d} z^{2}
$$

is the quadratic differential on $\mathcal{C}$. For our purposes, the important point to note is that the SW curve (2.29) can be written in the form (2.50) [39]. This construction will prove essential in what follows.

SW curves and dessins. As mentioned above, the SW curve $\Sigma$ is related to the quadratic differential $q$. Moving in the moduli space of the theory in question will alter the parameters in the SW curve, thereby altering the parameters in $q$ [9]. Following [47], it was found in [9] that at certain isolated points in the Coulomb branch $\mathcal{U}_{g, n}$, where $g$ is the genus of the Gaiotto curve $\mathcal{C}$ with $n$ marked points, $q$ is completely fixed and becomes a Strebel differential $q=\phi(t) \mathrm{d} t^{2}=\frac{\mathrm{d} \beta^{2}}{4 \pi^{2} \beta(t)(1-\beta(t))}$.

As examples for $\mathrm{SU}(2)$ with $N_{f}=4$, we will discuss 6 Strebel points in $\mathcal{U}_{g, n} \times \mathbb{R}^{n}$, for which the Belyi maps are presented in table 1. These six Belyi maps are those found in $[7,48]$ to be associated to the six genus zero, torsion-free, congruence subgroups of the

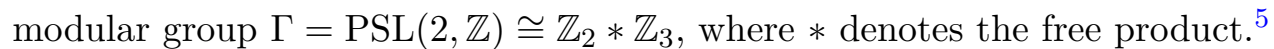

From the Belyi maps in table 1, we can compute the associated dessins as displayed in figure 2. The dessins d'enfants associated to each Strebel point of the generalised quiver theory in question turn out to have an interpretation as so-called ribbon graphs on the Gaiotto curve $\mathcal{C}$. For details, the readers are referred to $[9,47]$.

\footnotetext{
${ }^{5}$ For the background on the congruence subgroups of $\Gamma$, see appendix $C$. It remains an open question whether dessins associated to other subgroups of the modular group, perhaps of higher index, arise for other $\mathcal{N}=2$ generalised quiver theories in a parallel manner.
} 


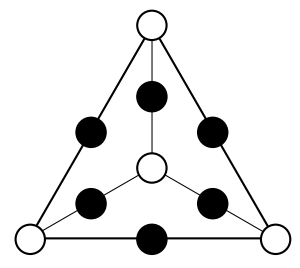

$\Gamma(3)$

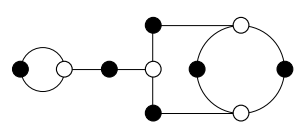

$\Gamma_{0}(6)$

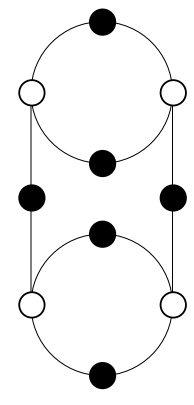

$\Gamma_{0}(4) \cap \Gamma(2)$

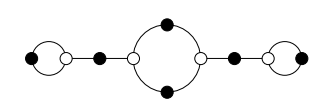

$\Gamma_{0}(8)$

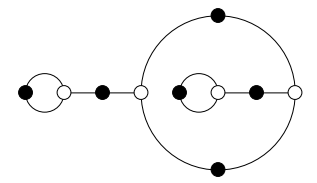

$\Gamma_{1}(5)$

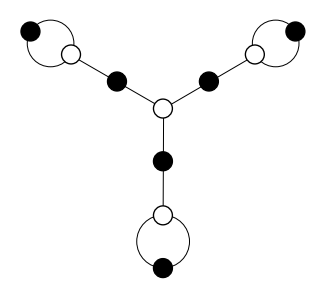

$\Gamma_{0}(9)$

Figure 2. The dessins d'enfants associated to the six Strebel points of the $\mathrm{SU}(2), N_{f}=4$ theory.

\section{From dessins to conformal blocks}

Let us now complete the cycle of the route map above by considering what gauge theory and CFT data we can obtain starting from these 6 dessins.

\subsection{The SU(2) with 4 flavours}

Given that all our graphs in figure 2 are drawn on the Riemann surface (genus zero) with 4 marked points (one for each face), we can naturally interpret these as Gaiotto curves [6, 9], and thence $\mathcal{N}=2$ gauge theories.

To begin, the Seiberg-Witten curve $\Sigma$ for the $\mathrm{SU}(2) N_{f}=4$ theory in algebraic form is standard [39]. For future convenience, we start with the SW curve of form (2.43) and write the SW differential as $[13,14]$

$$
\lambda_{\mathrm{SW}}=\frac{\sqrt{P_{4}(z)}}{z(z-1)(z-\zeta)} \mathrm{d} z, \quad P_{4}(z)=m_{0}^{2} \prod_{i=1}^{4}\left(z-\lambda_{i}\right)=m_{0}^{2} \sum_{i=0}^{4} z^{4-i} S_{i}
$$

under the substitution

$$
\begin{array}{rlrl}
\lambda_{\mathrm{SW}} & =v \mathrm{~d} z / z, & t & =z, \\
\mu_{1} & =m_{2}+m_{0}, \quad \mu_{2}=m_{2}-m_{0}, \quad \mu_{3}=m_{3}+m_{1}, \quad \mu_{4}=m_{3}-m_{1},
\end{array}
$$


The parameters $S_{i}$ are given in terms of the flavour mass and coupling parameters $m_{0,1,2,3}, \zeta, U \in \mathbb{C}$ so that $S_{0}=1$ for the top coefficient and

$$
\begin{aligned}
& m_{0}^{2} S_{1}=-\left(m_{0}^{2}+m_{2}^{2}(\zeta-1)+m_{0}^{2} \zeta+2 m_{2} m_{3} \zeta+(1+\zeta) U\right), \\
& m_{0}^{2} S_{2}=\left(m_{0}^{2}+m_{1}^{2}-m_{3}^{2}+2 m_{2} m_{3}\right) \zeta+m_{2}^{2}(\zeta-1) \zeta+2 m_{2} m_{3} \zeta^{2}+m_{3}^{2} \zeta^{2}+(1+\zeta)^{2} U, \\
& m_{0}^{2} S_{3}=-\left(\left(m_{1}^{2}-m_{3}^{2}\right) \zeta+\left(m_{1}^{2}+2 m_{2} m_{3}+m_{3}^{2}\right) \zeta^{2}+\zeta(1+\zeta) U\right), \\
& m_{0}^{2} S_{4}=m_{1}^{2} \zeta^{2} .
\end{aligned}
$$

Now the SW curve is of the form

$$
\begin{aligned}
z^{2}\left(v-\left(m_{0}+m_{2}\right)\right)\left(v-\left(m_{2}-m_{0}\right)\right)+z(1+\zeta) & \left(-v^{2}+\frac{2 \zeta}{(1+\zeta)}\left(m_{2}+m_{3}\right) v+U\right) \\
+ & \zeta\left(v-\left(m_{1}+m_{3}\right)\right)\left(v-\left(m_{3}-m_{1}\right)\right)=0 .
\end{aligned}
$$

On the other hand, the $S$-parameters can be written in terms of the $\lambda_{i}$ as standard symmetric polynomials,

$$
S_{k}=\sum_{1 \leq j_{1} \leq \ldots \leq j_{k} \leq 4} \lambda_{j_{1}} \ldots \lambda_{j_{k}} .
$$

Following appendix E.1, we can then write

$$
\frac{\mathrm{d} a(U)}{\mathrm{d} U}=-\frac{1}{\pi \mathrm{i}} \frac{1+\zeta}{m_{0} \sqrt{\left(\lambda_{2}-\lambda_{3}\right)\left(\lambda_{4}-\lambda_{1}\right)}} K(r),
$$

where

$$
r^{2}=\frac{\left(\lambda_{1}-\lambda_{2}\right)\left(\lambda_{3}-\lambda_{4}\right)}{\left(\lambda_{2}-\lambda_{3}\right)\left(\lambda_{4}-\lambda_{1}\right)},
$$

and $K(r)$ is the elliptic integral of the first kind. The right hand side of (3.6) implicitly depends on $U$, through $\lambda_{i}$ and thence $S_{i}$, thus we only need to integrate it to obtain $a(U)$ as a function of $U$, which could be a daunting task analytically.

Let us nevertheless attempt at some simplifications. First, we see that the right hand side depends only on the cross-terms in the four $\lambda_{i}$, which we will denote as $\lambda_{(i j)(k l)}=$ $\left(\lambda_{i}-\lambda_{j}\right)\left(\lambda_{k}-\lambda_{l}\right)$. Combining with (3.5), let us see whether these can be directly expressed in terms of $S_{i}$, and thence, in terms of $U$. This is a standard algebraic elimination problem and we readily find the following:

Lemma 3.1. Consider the monic cubic polynomial,

$$
\begin{aligned}
x^{3}+ & \left(-2 S_{2}^{2}+6 S_{1} S_{3}-24 S_{4}\right) x^{2} \\
& +\left(S_{2}^{4}-6 S_{1} S_{3} S_{2}^{2}+24 S_{4} S_{2}^{2}+9 S_{1}^{2} S_{3}^{2}+144 S_{4}^{2}-72 S_{1} S_{3} S_{4}\right) x+27 S_{4}^{2} S_{1}^{4}+4 S_{3}^{3} S_{1}^{3} \\
& -18 S_{2} S_{3} S_{4} S_{1}^{3}-144 S_{2} S_{4}^{2} S_{1}^{2}+4 S_{2}^{3} S_{4} S_{1}^{2}+6 S_{3}^{2} S_{4} S_{1}^{2}-18 S_{2} S_{3}^{3} S_{1}+192 S_{3} S_{4}^{2} S_{1} \\
& +80 S_{2}^{2} S_{3} S_{4} S_{1}+27 S_{3}^{4}-256 S_{4}^{3}+4 S_{2}^{3} S_{3}^{2}-S_{1}^{2} S_{2}^{2} S_{3}^{2}+128 S_{2}^{2} S_{4}^{2} \\
& -16 S_{2}^{4} S_{4}-144 S_{2} S_{3}^{2} S_{4} .
\end{aligned}
$$

Then the squares of the 3 cross-products

$$
x_{1}=\lambda_{(12)(34)}^{2}, x_{2}=\lambda_{(23)(41)}^{2}, x_{3}=\lambda_{(13)(24)}^{2}
$$

are the three roots of it. 
Of course, we can substitute the $S_{i}$ parameters in terms of the $m_{i}, \zeta, U$ parameters from (3.1), though the expression become too long to present here. Now, we have

$$
a\left(U_{0}\right)-a(U)=-\frac{1+\zeta}{m_{0} \pi \mathrm{i}} \int_{U}^{U_{0}} \frac{\mathrm{d} U^{\prime}}{\sqrt[4]{x_{2}\left(U^{\prime}\right)}} K\left(\frac{\sqrt[4]{x_{1}\left(U^{\prime}\right)}}{\sqrt[4]{x_{2}\left(U^{\prime}\right)}}\right)
$$

To determine the integral constant, we choose $U_{0}$ such that $a\left(U_{0}\right)=0$. We can find such $U_{0}$ by solving the discriminant of $P_{4}(z)$ where two branch points coincide and the $A$-cycle shrinks. ${ }^{6}$ Hence,

$$
a(U)=\frac{1+\zeta}{m_{0} \pi \mathrm{i}} \int_{U}^{U_{0}} \frac{\mathrm{d} U^{\prime}}{\sqrt[4]{x_{2}\left(U^{\prime}\right)}} K\left(\frac{\sqrt[4]{x_{1}\left(U^{\prime}\right)}}{\sqrt[4]{x_{2}\left(U^{\prime}\right)}}\right) .
$$

In general, when we integrate from some $U$ to $U_{0}$, the positions of branch points and cuts might change. Therefore, this is really a sum of integrals:

$$
\int_{U}^{U_{0}}=\int_{U}^{U_{1}}+\int_{U_{1}}^{U_{2}}+\cdots+\int_{U_{n-1}}^{U_{n}=U_{0}}
$$

such that $x_{i}$ does not change its expression for each integral on the right hand side.

Recall the definition of the Seiberg-Witten differential from (2.50), we have that

$$
\lambda_{\mathrm{SW}}^{2}=\phi_{\mathrm{SW}}(z) \mathrm{d} z^{2}
$$

is a quadratic differential. This is the above mentioned meromorphic quadratic differential on $\mathcal{C}$. Moving in the moduli space of the theory in question will alter the parameters in the Seiberg-Witten curve, thereby altering the parameters in $q$ (cf. [9]). Following [47], it was found in [9] that at certain isolated points in the Coulomb branch of the moduli space $\mathcal{U}_{g, n}$ of the gauge theory in question, where $g$ is the genus of the Gaiotto curve $\mathcal{C}$ with $n$ marked points, $q$ is completely fixed, which becomes a Strebel differential.

We therefore have two forms of the Strebel differentials, $\phi_{\beta}(t)$ coming from the dessin and $\phi_{\mathrm{SW}}(z)$ coming from the physics. Now, because dessins are rigid, they have no parameters. The insight of Belyi and Grothendieck is precisely that the maps $\beta$ have parameters fixed at very special algebraic points in moduli space. Thus, $\phi_{\beta}(t)$ is of a particular form, as a rational function in $t$ with fixed algebraic coefficients.

On the other hand $\phi_{\mathrm{SW}}(z)$ from the gauge theory has parameters which we saw earlier, corresponding to masses, couplings etc. Therefore, up to redefinition of the variables $(t, z)$ and identifying $\phi_{\mathrm{SW}}(z)$ and $\phi_{\beta}(t)$ it is natural to ask how the special values of the parameters from the dessin perspective fix the physical parameters in the gauge theory and if a dessin implicates any interesting physical theory.

We have now introduced all the necessary dramatis personae of our tale and our strategy is thus clear. There are also some further details that we should be careful about in the calculations. We will work through an example in detail to illustrate them in the following subsection.

\footnotetext{
${ }^{6}$ Alternatively, we may also integrate from $U$ to $\infty$ as the large $U$ behaviour can be determined as in appendix E.2.
} 


\section{$3.2 \quad$ Example: $\Gamma(3)$}

Let us take the dessin for $\Gamma(3)$, whose Belyi map is

$$
\beta(t)=\frac{t^{3}(t+6)^{3}\left(t^{2}-6 t+36\right)^{3}}{1728(t-3)^{3}\left(t^{2}+3 t+9\right)^{3}} .
$$

We can readily get the pre-images of 0,1 and $\infty$ :

\begin{tabular}{|c|c|c|}
\hline & Pre-image & Ramification \\
\hline$\beta^{-1}(0)$ & -6 & 3 \\
& 0 & 3 \\
& $3-3 i \sqrt{3}$ & 3 \\
& $3+3 i \sqrt{3}$ & 3 \\
\hline$\beta^{-1}(1)$ & $3(1-\sqrt{3})$ & 2 \\
& $3(1+\sqrt{3})$ & 2 \\
& $\left(\frac{3}{2}+\frac{3 i}{2}\right)(\sqrt{3}+(-2-i))$ & 2 \\
& $\left(-\frac{3}{2}-\frac{3 i}{2}\right)(\sqrt{3}+(2+i))$ & 2 \\
& $\frac{1}{2}((-3+9 i)-(3-3 i) \sqrt{3})$ & 2 \\
& $\frac{1}{2}((-3+9 i)+(3-3 i) \sqrt{3})$ & 2 \\
\hline$\beta^{-1}(\infty)$ & $\infty$ & 3 \\
& 3 & 3 \\
& $-\frac{3}{2} i(\sqrt{3}-i)$ & 3 \\
& $\frac{3}{2} i(\sqrt{3}+i)$ & 3 \\
\hline
\end{tabular}

We can construct the corresponding dessin as in figure 2. Subsequently, using (2.48), we see that the Strebel differential is $q=\phi_{\beta}(t) \mathrm{d} t^{2}$, where

$$
\phi_{\beta}(t)=-\frac{9 t\left(t^{3}+216\right)}{4 \pi^{2}\left(t^{3}-27\right)^{2}} .
$$

We have marked $\phi$ with a subscript $\beta$ to emphasize its dessin origin. On the other side, we have the Seiberg-Witten curve and quadratic differential for SU(2) with $N_{f}=4$ from (3.1) and (3.13), to be

$$
\begin{aligned}
\phi_{\mathrm{SW}}(z)= & \frac{P_{4}(z)}{(z(z-1)(z-\zeta))^{2}}, \quad \text { where } \\
P_{4}(z)= & z^{4} m_{0}^{2}-z^{3}\left(m_{0}^{2}+m_{2}^{2}(\zeta-1)+m_{0}^{2} \zeta+2 m_{2} m_{3} \zeta+(1+\zeta) U\right) \\
& +z^{2}\left(\left(m_{0}^{2}+m_{1}^{2}-m_{3}^{2}+2 m_{2} m_{3}\right) \zeta+m_{2}^{2}(\zeta-1) \zeta+2 m_{2} m_{3} \zeta^{2}+m_{3}^{2} \zeta^{2}+(1+\zeta)^{2} U\right) \\
& -z\left(\left(m_{1}^{2}-m_{3}^{2}\right) \zeta+\left(m_{1}^{2}+2 m_{2} m_{3}+m_{3}^{2}\right) \zeta^{2}+\zeta(1+\zeta) U\right)+m_{1}^{2} \zeta^{2} .
\end{aligned}
$$

Here, likewise we have marked $\phi$ with a subscript "SW" to emphasize its SeibergWitten origin. We have also explicitly written the differential coming from the SeibergWitten side in terms of the parameters $m_{0,1,2,3}, \zeta, U$. 
We need to match $(3.16)$ with $(3.17)$, up to an $\operatorname{PGL}(2, \mathbb{C})$ transformation on the complex variable $z$. The reason for this is that we are dealing in this example with a quadratic differential on the sphere. For curves of higher genus, such $\operatorname{PGL}(2, \mathbb{C})$ transformations are generically not permitted, as they will not preserve the structure of the poles and zeros of the quadratic differential.

We can therefore write

$$
z=\frac{a t+b}{c t+d}, \quad a, b, c, d \in \mathbb{C}
$$

and solve for complex coefficients $a, b, c, d$ as well as the parameters $m_{0,1,2,3}, \zeta, U$ so that we have identically for all $t$ that

$$
\phi_{\beta}(t)=\phi_{\mathrm{SW}}\left(\frac{a t+b}{c t+d}\right) .
$$

There are actually continuous families of $2 \times 2$ matrices solving this equation for a given dessin. As the elliptic curve is the same up to an overall factor, it turns out that each continuous family would simply scale the SW differential by $\phi_{\mathrm{SW}} \rightarrow k^{2} \phi_{\mathrm{SW}}$ with $k^{2} \in \mathbb{R}$, where the square comes from the $\lambda_{\mathrm{SW}}^{2}$ in the differential. Obviously, equating the numerators of $\phi_{\beta}$ and $\phi_{\mathrm{SW}}$ as well as equating their denominators would give a solution. For future convenience, such solution will be referred to as the "basic" values of the parametrization. Then other parametrizations would simply follow

$$
\phi_{\mathrm{SW}}=k^{2} \phi_{\mathrm{SW}, \mathrm{basic}} .
$$

There are two points we should pay attention to:

- As we will try to relate this to minimal models, due to modular invariance, we can only allow primary states with pure imaginary charges [49]. Recall the AGT relation (2.11), which in terms of $m_{i}$ is

$$
\frac{m_{0}}{\sqrt{\epsilon_{1} \epsilon_{2}}}+\frac{Q}{2}=\alpha_{4}, \frac{m_{1}}{\sqrt{\epsilon_{1} \epsilon_{2}}}+\frac{Q}{2}=\alpha_{1}, \frac{m_{2}}{\sqrt{\epsilon_{1} \epsilon_{2}}}=\alpha_{3}, \frac{m_{3}}{\sqrt{\epsilon_{1} \epsilon_{2}}}=\alpha_{2}, \frac{a}{\sqrt{\epsilon_{1} \epsilon_{2}}}+\frac{Q}{2}=\alpha_{\text {int }} .
$$

In fact, $\epsilon_{1,2}$ are not completely free once $Q=\left(\epsilon_{1}+\epsilon_{2}\right) / \sqrt{\epsilon_{1} \epsilon_{2}}$ is chosen. Moreover, to have real conformal dimensions, $m_{i}$ 's and $a$ should only be real or pure imaginary (depending on $\epsilon_{1,2}$ ). This is also the reason why $k^{2}$ should be real.

- One may easily check that an SW differential/elliptic curve would have the same $j$ invariant under $\phi \rightarrow k^{2} \phi$. As a result, the parameters, based on their mass dimensions or by looking at $P_{4}(z)$ and $a(U)$, would follow

$$
\begin{aligned}
& m_{i} \rightarrow k m_{i}, \quad a \rightarrow k a, \quad \epsilon_{i} \rightarrow k \epsilon_{i}, \quad U \rightarrow k^{2} U ; \\
& \zeta \rightarrow \zeta, \quad \alpha_{i, \text { int }} \rightarrow \alpha_{i, \text { int }}, \quad Q \rightarrow Q \text {. }
\end{aligned}
$$

Therefore, rather than discrete parameters, we would have families of differentials. Importantly, we can see that the coupling $\zeta$ is invariant. Following the AGT map, the dimensionless CFT parameters, $\alpha_{i \text {,int }}$ and $Q$, are also invariant under the scaling though we still have the freedom to choose $\sqrt{\epsilon_{1} \epsilon_{2}}$. 
Now expanding the above and setting all the coefficients of $t$ to vanish identically gives a complicated polynomial system in $\left(a, b, c, d, m_{0,1,2,3}, \zeta, U\right)$ for which one can find many solutions. For example, the following constitutes a solution (with $k^{2}=1$ ),

$$
m_{0}=-m_{1}=m_{2}=-m_{3}=\frac{1}{2 \sqrt{3} \pi}, \quad \zeta=\frac{1}{2}+\frac{\mathrm{i} \sqrt{3}}{2}, \quad U=\frac{1}{9 \pi^{2}}
$$

with $(a, b, c, d)=\left(\frac{1+\mathrm{i}}{\sqrt{2} 3^{3 / 4}}, \mathrm{i}\left(\frac{\sqrt[4]{3}}{2 \sqrt{2}}-\frac{3^{3 / 4}}{2 \sqrt{2}}\right)+\frac{\sqrt[4]{3}}{2 \sqrt{2}}+\frac{3^{3 / 4}}{2 \sqrt{2}}, 0, \frac{(1-\mathrm{i}) 3^{3 / 4}}{\sqrt{2}}\right)$. The numerator of the SW differential takes the form

$$
P_{4}(z)=-\frac{6 z^{4}-4 \mathrm{i}(\sqrt{3}-3 \mathrm{i}) z^{3}+(6+6 \mathrm{i} \sqrt{3}) z^{2}-8 \mathrm{i} \sqrt{3} z+3 \mathrm{i} \sqrt{3}-3}{72 \pi^{2}} .
$$

We now need the roots $\lambda_{i}$ of $P_{4}(z)$ as given in (3.1):

$$
z^{4}+\left(-2-\frac{2 \mathrm{i}}{\sqrt{3}}\right) z^{3}+(1+\mathrm{i} \sqrt{3}) z^{2}-\frac{4 \mathrm{i}}{\sqrt{3}} z+\frac{1}{2} \mathrm{i}(\sqrt{3}+\mathrm{i})=\prod_{i=1}^{4}\left(z-\lambda_{i}\right) .
$$

The SW curve itself is genus 1 and is in fact an elliptic curve. We can recast (3.17) as

$$
\begin{aligned}
y^{2}= & z^{4} m_{0}^{2}-z^{3}\left(m_{0}^{2}+m_{2}^{2}(\zeta-1)+m_{0}^{2} \zeta+2 m_{2} m_{3} \zeta+(1+\zeta) U\right) \\
& +z^{2}\left(\left(m_{0}^{2}+m_{1}^{2}-m_{3}^{2}+2 m_{2} m_{3}\right) \zeta+m_{2}^{2}(\zeta-1) \zeta+2 m_{2} m_{3} \zeta^{2}+m_{3}^{2} \zeta^{2}+(1+\zeta)^{2} U\right) \\
& -z\left(\left(m_{1}^{2}-m_{3}^{2}\right) \zeta+\left(m_{1}^{2}+2 m_{2} m_{3}+m_{3}^{2}\right) \zeta^{2}+\zeta(1+\zeta) U\right)+m_{1}^{2} \zeta^{2},
\end{aligned}
$$

where the redefinition $y^{2}=(z(z-1)(z-\zeta))^{2} \phi_{\mathrm{SW}}(z)=P_{4}(z)$ is used. Following appendix D, as one may check, the $j$-invariant we get from the parameterization (3.23) agrees with the one directly from the Strebel differential (3.16):

$$
j=0 .
$$

Indeed, $j=0$ corresponds to a special elliptic curve with $\mathbb{Z} / 3 \mathbb{Z}$-symmetry, much like the dessin for $\Gamma(3)$ itself.

In this case, we can integrate (3.11) numerically to obtain $a(U)=\frac{1}{3 \sqrt{3} \pi} \cdot{ }^{7}$ Now we can use the AGT relation (3.21) to get the parametrizations for CBs. If we take $Q=0$, we have

$$
\alpha_{1}=\alpha_{2}=-\alpha_{3}=-\alpha_{4}=\frac{\mathrm{i}}{2 \sqrt{3} \pi}, \alpha_{\mathrm{int}}=\frac{\mathrm{i}}{3 \sqrt{3} \pi},
$$

where we have chosen $-\epsilon_{1}=\epsilon_{2}=1$ as an example.

We can also have pure imaginary $m_{i}$ 's and $a$ for the above example such as

$$
m_{0}=-m_{1}=m_{2}=-m_{3}=\frac{\mathrm{i}}{2 \sqrt{3} \pi}, \zeta=\frac{1}{2}+\frac{\mathrm{i} \sqrt{3}}{2}, U=-\frac{1}{9 \pi^{2}}, a=\frac{\mathrm{i}}{3 \sqrt{3} \pi} .
$$

Then we can still get the same CFT parameters for $Q=0$ as in (3.28) with the choice $-\epsilon_{1}=\epsilon_{2}=\mathrm{i}$.

\footnotetext{
${ }^{7}$ Numerical integration would often give decimals rather than precise values. For instance, here we get $a \approx 0.06125877$. In some cases like here, we give exact values for $a$ with the help of minimal models. This can be done by checking multiple minimal models and finding certain $\epsilon_{1,2}$ so that $\Delta_{i}$ would fit into their Kac tables. Then the correct closed form of $a$ can be obtained if $\Delta_{\text {int }}$ also lives in these Kac tables for all these minimal models. (To get the correct CBs under AGT map, we further need the fusion rule, but even if the corresponding $\Delta_{\text {int }}$ does not satisfy the fusion rule for some CB, this could still be regarded as a verfication of fine-tuning $a$ as long as $\Delta_{\text {int }}$, along with $\Delta_{i}$, belongs to the Kac table.)
} 


\begin{tabular}{|c|c|c|c|c|c|c|c|}
\hline$\zeta=\mathrm{e}^{2 \pi \mathrm{i} \tau}$ & $m_{0}$ & $m_{1}$ & $m_{2}$ & $m_{3}$ & $U$ & $\sum_{i} m_{i}$ & $a=\frac{\alpha_{\text {int }}}{\sqrt{\epsilon_{1} \epsilon_{2}}}+\frac{Q}{2}$ \\
\hline \multirow{5}{*}{$\frac{1}{2}(1-i \sqrt{3})$} & $-\frac{i}{2 \sqrt{3} \pi}$ & $-\frac{i}{2 \sqrt{3} \pi}$ & $\frac{i}{2 \sqrt{3} \pi}$ & $-\frac{i}{2 \sqrt{3} \pi}$ & $-\frac{1}{9 \pi^{2}}$ & $\frac{i}{\sqrt{3} \pi}$ & $-\frac{i}{3 \sqrt{3} \pi}$ \\
\cline { 2 - 8 } & $-\frac{i}{2 \sqrt{3} \pi}$ & $\frac{i}{2 \sqrt{3} \pi}$ & $-\frac{i}{2 \sqrt{3} \pi}$ & $\frac{i}{2 \sqrt{3} \pi}$ & $-\frac{1}{9 \pi^{2}}$ & 0 & $-\frac{i}{3 \sqrt{3} \pi}$ \\
\cline { 2 - 8 } & $\frac{i}{2 \sqrt{3} \pi}$ & $-\frac{i}{2 \sqrt{3} \pi}$ & $\frac{i}{2 \sqrt{3} \pi}$ & $-\frac{i}{2 \sqrt{3} \pi}$ & $-\frac{1}{9 \pi^{2}}$ & 0 & $\frac{i}{3 \sqrt{3} \pi}$ \\
\cline { 2 - 8 } & $\frac{i}{2 \sqrt{3} \pi}$ & $\frac{i}{2 \sqrt{3} \pi}$ & $-\frac{i}{2 \sqrt{3} \pi}$ & $\frac{i}{2 \sqrt{3} \pi}$ & $-\frac{1}{9 \pi^{2}}$ & $-\frac{i}{\sqrt{3} \pi}$ & $\frac{i}{3 \sqrt{3} \pi}$ \\
\hline \multirow{5}{*}{$\frac{1}{2}(1+i \sqrt{3})$} & $-\frac{i}{2 \sqrt{3} \pi}$ & $-\frac{i}{2 \sqrt{3} \pi}$ & $\frac{i}{2 \sqrt{3} \pi}$ & $-\frac{i}{2 \sqrt{3} \pi}$ & $-\frac{1}{9 \pi^{2}}$ & $\frac{i}{\sqrt{3} \pi}$ & $\frac{i}{3 \sqrt{3} \pi}$ \\
\cline { 2 - 8 } & $-\frac{i}{2 \sqrt{3} \pi}$ & $\frac{i}{2 \sqrt{3} \pi}$ & $-\frac{i}{2 \sqrt{3} \pi}$ & $\frac{i}{2 \sqrt{3} \pi}$ & $-\frac{1}{9 \pi^{2}}$ & 0 & $\frac{i}{3 \sqrt{3} \pi}$ \\
\cline { 2 - 8 } & $\frac{i}{2 \sqrt{3} \pi}$ & $-\frac{i}{2 \sqrt{3} \pi}$ & $\frac{i}{2 \sqrt{3} \pi}$ & $-\frac{i}{2 \sqrt{3} \pi}$ & $-\frac{1}{9 \pi^{2}}$ & 0 & $-\frac{i}{3 \sqrt{3} \pi}$ \\
\cline { 2 - 8 } & $\frac{i}{2 \sqrt{3} \pi}$ & $\frac{i}{2 \sqrt{3} \pi}$ & $-\frac{i}{2 \sqrt{3} \pi}$ & $\frac{i}{2 \sqrt{3} \pi}$ & $-\frac{1}{9 \pi^{2}}$ & $-\frac{i}{\sqrt{3} \pi}$ & $-\frac{i}{3 \sqrt{3} \pi}$ \\
\hline
\end{tabular}

Table 2. The parameters obtained from $\Gamma(3)$. Using (3.21), we can get the values for $\alpha_{i}$ 's.

\subsection{Matching parameters}

Here, we report all parameters from the six dessins in tables 2-7. Notice that we are only giving solutions coming from $( \pm) \phi_{\mathrm{SW} \text {,basic }}$ with pure imaginary $m_{i}$ 's and $a$. There is actually a family for each parametrization following (3.22).

As the size of the table increases, we will give a more compact version for the remaining cases below. For each $\zeta$, there are usually $2^{4}=16$ possibilities. For $a$, as the sign of $a$ only depends on the sign of $m_{0}$ (in the following sense), " \pm " in $a$ means that $a$ has the same sign as $m_{0}$ while " $\mp$ " in $a$ indicates that $m_{0}$ and $a$ have opposite signs.

Based on the above calculations, there are some remarks we can make:

- One may check that the elliptic curves parametrized by these $m_{i}, \zeta$ and $U$ have the same $j$-invariants as in table 8 for the six Belyi maps. Moreover, there are two cases with $\zeta=(1 \pm i \sqrt{3}) / 2$, which are the cusp points for the fundamental diagram of $\mathrm{SL}(2, \mathbb{Z})$. They are exactly the dessins whose Belyi maps have $j$-invariant 0 .

- It is obvious that for each dessin, the parametrizations for different $\zeta$ 's are related by triality

$$
\zeta \leftrightarrow \zeta^{\prime}=\frac{1}{\zeta} \leftrightarrow \zeta^{\prime \prime}=1-\zeta
$$

This is explicitly listed in table 9. Modular invariance of the curve also leads to the following transformations of mass parameters:

$$
\begin{array}{rlrl}
\zeta & \leftrightarrow \frac{1}{\zeta}: & \left(m_{0}, m_{1}, m_{2}, m_{3}\right) & \leftrightarrow \frac{1}{|\zeta|}\left(m_{0}, m_{1}, m_{3}, m_{2}\right) ; \\
\zeta & \leftrightarrow 1-\zeta: \quad\left(m_{0}, m_{1}, m_{2}, m_{3}\right) \leftrightarrow\left(m_{0}, m_{2}, m_{1}, m_{3}\right) .
\end{array}
$$

In particular, the two rows for $\Gamma_{1}(5)$ are also related by triality: $1-\left(\frac{125}{2}+\frac{55}{2} \sqrt{5}\right)=$ $-\frac{123}{2}-\frac{55}{2} \sqrt{5}$. 


\begin{tabular}{|c|c|c|c|c|c|c|c|}
\hline$\zeta=\mathrm{e}^{2 \pi \mathrm{i} \tau}$ & $m_{0}$ & $m_{1}$ & $m_{2}$ & $m_{3}$ & $U$ & $\sum_{i} m_{i}$ & $a$ \\
\hline \multirow{16}{*}{$\frac{1}{2}$} & $-\frac{i}{8 \pi}$ & $-\frac{i}{4 \pi}$ & $-\frac{i}{4 \pi}$ & $-\frac{i}{8 \pi}$ & $-\frac{1}{192 \pi^{2}}$ & $\frac{3 i}{4 \pi}$ & $\frac{i}{8 \pi}$ \\
\hline & $\frac{i}{8 \pi}$ & $-\frac{i}{4 \pi}$ & $-\frac{i}{4 \pi}$ & $-\frac{i}{8 \pi}$ & $-\frac{1}{192 \pi^{2}}$ & $\frac{i}{2 \pi}$ & $-\frac{i}{8 \pi}$ \\
\hline & $-\frac{i}{8 \pi}$ & $-\frac{i}{4 \pi}$ & $-\frac{i}{4 \pi}$ & $\frac{i}{8 \pi}$ & $-\frac{3}{64 \pi^{2}}$ & $\frac{i}{2 \pi}$ & $\frac{i}{8 \pi}$ \\
\hline & $-\frac{i}{8 \pi}$ & $\frac{i}{4 \pi}$ & $-\frac{i}{4 \pi}$ & $-\frac{i}{8 \pi}$ & $-\frac{1}{192 \pi^{2}}$ & $\frac{i}{4 \pi}$ & $\frac{i}{8 \pi}$ \\
\hline & $\frac{i}{8 \pi}$ & $-\frac{i}{4 \pi}$ & $-\frac{i}{4 \pi}$ & $\frac{i}{8 \pi}$ & $-\frac{3}{64 \pi^{2}}$ & $\frac{i}{4 \pi}$ & $-\frac{i}{8 \pi}$ \\
\hline & $-\frac{i}{8 \pi}$ & $-\frac{i}{4 \pi}$ & $\frac{i}{4 \pi}$ & $-\frac{i}{8 \pi}$ & $-\frac{3}{64 \pi^{2}}$ & $\frac{i}{4 \pi}$ & $\frac{i}{8 \pi}$ \\
\hline & $\frac{i}{8 \pi}$ & $\frac{i}{4 \pi}$ & $-\frac{i}{4 \pi}$ & $-\frac{i}{8 \pi}$ & $-\frac{1}{192 \pi^{2}}$ & 0 & $-\frac{i}{8 \pi}$ \\
\hline & $-\frac{i}{8 \pi}$ & $\frac{i}{4 \pi}$ & $-\frac{i}{4 \pi}$ & $\frac{i}{8 \pi}$ & $-\frac{3}{64 \pi^{2}}$ & 0 & $\frac{i}{8 \pi}$ \\
\hline & $\frac{i}{8 \pi}$ & $-\frac{i}{4 \pi}$ & $\frac{i}{4 \pi}$ & $-\frac{i}{8 \pi}$ & $-\frac{3}{64 \pi^{2}}$ & 0 & $-\frac{i}{8 \pi}$ \\
\hline & $-\frac{i}{8 \pi}$ & $-\frac{i}{4 \pi}$ & $\frac{i}{4 \pi}$ & $\frac{i}{8 \pi}$ & $-\frac{1}{192 \pi^{2}}$ & 0 & $\frac{i}{8 \pi}$ \\
\hline & $\frac{i}{8 \pi}$ & $\frac{i}{4 \pi}$ & $-\frac{i}{4 \pi}$ & $\frac{i}{8 \pi}$ & $-\frac{3}{64 \pi^{2}}$ & $-\frac{i}{4 \pi}$ & $-\frac{i}{8 \pi}$ \\
\hline & $-\frac{i}{8 \pi}$ & $\frac{i}{4 \pi}$ & $\frac{i}{4 \pi}$ & $-\frac{i}{8 \pi}$ & $-\frac{3}{64 \pi^{2}}$ & $-\frac{i}{4 \pi}$ & $\frac{i}{8 \pi}$ \\
\hline & $\frac{i}{8 \pi}$ & $-\frac{i}{4 \pi}$ & $\frac{i}{4 \pi}$ & $\frac{i}{8 \pi}$ & $-\frac{1}{192 \pi^{2}}$ & $-\frac{i}{4 \pi}$ & $-\frac{i}{8 \pi}$ \\
\hline & $\frac{i}{8 \pi}$ & $\frac{i}{4 \pi}$ & $\frac{i}{4 \pi}$ & $-\frac{i}{8 \pi}$ & $-\frac{3}{64 \pi^{2}}$ & $-\frac{i}{2 \pi}$ & $-\frac{i}{8 \pi}$ \\
\hline & $-\frac{i}{8 \pi}$ & $\frac{i}{4 \pi}$ & $\frac{i}{4 \pi}$ & $\frac{i}{8 \pi}$ & $-\frac{1}{192 \pi^{2}}$ & $-\frac{i}{2 \pi}$ & $\frac{i}{8 \pi}$ \\
\hline & $\frac{i}{8 \pi}$ & $\frac{i}{4 \pi}$ & $\frac{i}{4 \pi}$ & $\frac{i}{8 \pi}$ & $-\frac{1}{192 \pi^{2}}$ & $-\frac{3 i}{4 \pi}$ & $\frac{i}{8 \pi}$ \\
\hline \multirow{16}{*}{2} & $-\frac{i}{4 \pi}$ & $-\frac{i}{2 \pi}$ & $-\frac{i}{4 \pi}$ & $-\frac{i}{2 \pi}$ & $\frac{1}{6 \pi^{2}}$ & $\frac{3 i}{2 \pi}$ & $\frac{i}{2 \pi}$ \\
\hline & $\frac{i}{4 \pi}$ & $-\frac{i}{2 \pi}$ & $-\frac{i}{4 \pi}$ & $-\frac{i}{2 \pi}$ & $\frac{1}{6 \pi^{2}}$ & $\frac{i}{\pi}$ & $-\frac{i}{2 \pi}$ \\
\hline & $-\frac{i}{4 \pi}$ & $-\frac{i}{2 \pi}$ & $\frac{i}{4 \pi}$ & $-\frac{i}{2 \pi}$ & $-\frac{1}{6 \pi^{2}}$ & $\frac{i}{\pi}$ & $\frac{i}{2 \pi}$ \\
\hline & $\frac{i}{4 \pi}$ & $-\frac{i}{2 \pi}$ & $\frac{i}{4 \pi}$ & $-\frac{i}{2 \pi}$ & $-\frac{1}{6 \pi^{2}}$ & $\frac{i}{2 \pi}$ & $-\frac{i}{2 \pi}$ \\
\hline & $-\frac{i}{4 \pi}$ & $-\frac{i}{2 \pi}$ & $-\frac{i}{4 \pi}$ & $\frac{i}{2 \pi}$ & $-\frac{1}{6 \pi^{2}}$ & $\frac{i}{2 \pi}$ & $\frac{i}{2 \pi}$ \\
\hline & $-\frac{i}{4 \pi}$ & $\frac{i}{2 \pi}$ & $-\frac{i}{4 \pi}$ & $-\frac{i}{2 \pi}$ & $\frac{1}{6 \pi^{2}}$ & $\frac{i}{2 \pi}$ & $\frac{i}{2 \pi}$ \\
\hline & $\frac{i}{4 \pi}$ & $\frac{i}{2 \pi}$ & $-\frac{i}{4 \pi}$ & $-\frac{i}{2 \pi}$ & $\frac{1}{6 \pi^{2}}$ & 0 & $-\frac{i}{2 \pi}$ \\
\hline & $-\frac{i}{4 \pi}$ & $\frac{i}{2 \pi}$ & $\frac{i}{4 \pi}$ & $-\frac{i}{2 \pi}$ & $-\frac{1}{6 \pi^{2}}$ & 0 & $\frac{i}{2 \pi}$ \\
\hline & $\frac{i}{4 \pi}$ & $-\frac{i}{2 \pi}$ & $-\frac{i}{4 \pi}$ & $\frac{i}{2 \pi}$ & $-\frac{1}{6 \pi^{2}}$ & 0 & $-\frac{i}{2 \pi}$ \\
\hline & $-\frac{i}{4 \pi}$ & $-\frac{i}{2 \pi}$ & $\frac{i}{4 \pi}$ & $\frac{i}{2 \pi}$ & $\frac{1}{6 \pi^{2}}$ & 0 & $\frac{i}{2 \pi}$ \\
\hline & $\frac{i}{4 \pi}$ & $-\frac{i}{2 \pi}$ & $\frac{i}{4 \pi}$ & $\frac{i}{2 \pi}$ & $\frac{1}{6 \pi^{2}}$ & $-\frac{i}{2 \pi}$ & $-\frac{i}{2 \pi}$ \\
\hline & $\frac{i}{4 \pi}$ & $\frac{i}{2 \pi}$ & $\frac{i}{4 \pi}$ & $-\frac{i}{2 \pi}$ & $-\frac{1}{6 \pi^{2}}$ & $-\frac{i}{2 \pi}$ & $-\frac{i}{2 \pi}$ \\
\hline & $-\frac{i}{4 \pi}$ & $\frac{i}{2 \pi}$ & $-\frac{i}{4 \pi}$ & $\frac{i}{2 \pi}$ & $-\frac{1}{6 \pi^{2}}$ & $-\frac{i}{2 \pi}$ & $\frac{i}{2 \pi}$ \\
\hline & $\frac{i}{4 \pi}$ & $\frac{i}{2 \pi}$ & $-\frac{i}{4 \pi}$ & $\frac{i}{2 \pi}$ & $-\frac{1}{6 \pi^{2}}$ & $-\frac{i}{\pi}$ & $-\frac{i}{2 \pi}$ \\
\hline & $-\frac{i}{4 \pi}$ & $\frac{i}{2 \pi}$ & $\frac{i}{4 \pi}$ & $\frac{i}{2 \pi}$ & $\frac{1}{6 \pi^{2}}$ & $-\frac{i}{\pi}$ & $\frac{i}{2 \pi}$ \\
\hline & $\frac{i}{4 \pi}$ & $\frac{i}{2 \pi}$ & $\frac{i}{4 \pi}$ & $\frac{i}{2 \pi}$ & $\frac{1}{6 \pi^{2}}$ & $-\frac{3 i}{2 \pi}$ & $-\frac{i}{2 \pi}$ \\
\hline \multirow{8}{*}{-1} & $-\frac{i}{4 \pi}$ & $-\frac{i}{4 \pi}$ & $-\frac{i}{2 \pi}$ & $\frac{i}{2 \pi}$ & \multirow{8}{*}{$\begin{array}{c}\text { Any } \\
\text { value }^{(*)}\end{array}$} & $\frac{i}{2 \pi}$ & \multirow{8}{*}{0} \\
\hline & $-\frac{i}{4 \pi}$ & $-\frac{i}{4 \pi}$ & $\frac{i}{2 \pi}$ & $-\frac{i}{2 \pi}$ & & $\frac{i}{2 \pi}$ & \\
\hline & $\frac{i}{4 \pi}$ & $-\frac{i}{4 \pi}$ & $-\frac{i}{2 \pi}$ & $\frac{i}{2 \pi}$ & & 0 & \\
\hline & $\frac{i}{4 \pi}$ & $-\frac{i}{4 \pi}$ & $\frac{i}{2 \pi}$ & $-\frac{i}{2 \pi}$ & & 0 & \\
\hline & $-\frac{i}{4 \pi}$ & $\frac{i}{4 \pi}$ & $-\frac{i}{2 \pi}$ & $\frac{i}{2 \pi}$ & & 0 & \\
\hline & $-\frac{i}{4 \pi}$ & $\frac{i}{4 \pi}$ & $\frac{i}{2 \pi}$ & $-\frac{i}{2 \pi}$ & & 0 & \\
\hline & $\frac{i}{4 \pi}$ & $\frac{i}{4 \pi}$ & $-\frac{i}{2 \pi}$ & $\frac{i}{2 \pi}$ & & $-\frac{i}{2 \pi}$ & \\
\hline & $\frac{i}{4 \pi}$ & $\frac{i}{4 \pi}$ & $\frac{i}{2 \pi}$ & $-\frac{i}{2 \pi}$ & & $-\frac{i}{2 \pi}$ & \\
\hline
\end{tabular}

$(*)$ : here, any complex number can be a basic value for $U$ since all the terms of $U$ in $P_{4}(z)$ contain $(1+\zeta)$ as well. Moreover, the integral for $a$ always vanishes.

Table 3. The parameters obtained from $\Gamma_{0}(4) \cap \Gamma(2)$. Using (3.21), we can get the values for $\alpha_{i}$ 's. 


\begin{tabular}{|c|c|c|c|c|c|c|}
\hline$\zeta=\mathrm{e}^{2 \pi \mathrm{i} \tau}$ & $m_{0}$ & $m_{1}$ & $m_{2}$ & $m_{3}$ & $U$ & $a$ \\
\hline \multirow{4}{*}{$\frac{1}{2}-\frac{11}{50} \sqrt{5}$} & \multirow[t]{2}{*}{$\pm \frac{i \sqrt{5}}{50 \pi}$} & \multirow[t]{2}{*}{$\pm \frac{i \sqrt{5}}{10 \pi}$} & $\frac{i \sqrt{5}}{10 \pi}$ & $\frac{i \sqrt{5}}{50 \pi}$ & \multirow[t]{2}{*}{$\frac{(-607+85 \sqrt{5})}{62750 \pi^{2}}$} & \multirow[t]{2}{*}{$\pm \frac{i \sqrt{5}}{25 \pi}$} \\
\hline & & & $-\frac{i \sqrt{5}}{10 \pi}$ & $-\frac{i \sqrt{5}}{50 \pi}$ & & \\
\hline & \multirow[t]{2}{*}{$\pm \frac{i \sqrt{5}}{50 \pi}$} & \multirow[t]{2}{*}{$\pm \frac{i \sqrt{5}}{10 \pi}$} & $\frac{i \sqrt{5}}{10 \pi}$ & $-\frac{i \sqrt{5}}{50 \pi}$ & \multirow[t]{2}{*}{$\frac{9(-69+20 \sqrt{5})}{31375 \pi^{2}}$} & \multirow[t]{2}{*}{$\mp \frac{i \sqrt{5}}{25 \pi}$} \\
\hline & & & $-\frac{i \sqrt{5}}{10 \pi}$ & $\frac{i \sqrt{5}}{50 \pi}$ & & \\
\hline \multirow{4}{*}{$\frac{1}{2}+\frac{11}{50} \sqrt{5}$} & \multirow[t]{2}{*}{$\pm \frac{i \sqrt{5}}{50 \pi}$} & \multirow[t]{2}{*}{$\pm \frac{i \sqrt{5}}{10 \pi}$} & $\frac{i \sqrt{5}}{10 \pi}$ & $\frac{i \sqrt{5}}{50 \pi}$ & \multirow[t]{2}{*}{$\frac{(-607-85 \sqrt{5})}{62750 \pi^{2}}$} & \multirow[t]{2}{*}{$\mp \frac{i \sqrt{5}}{25 \pi}$} \\
\hline & & & $-\frac{i \sqrt{5}}{10 \pi}$ & $-\frac{i \sqrt{5}}{50 \pi}$ & & \\
\hline & \multirow[t]{2}{*}{$\pm \frac{i \sqrt{5}}{50 \pi}$} & \multirow[t]{2}{*}{$\pm \frac{i \sqrt{5}}{10 \pi}$} & $\frac{i \sqrt{5}}{10 \pi}$ & $-\frac{i \sqrt{5}}{50 \pi}$ & \multirow[t]{2}{*}{$\frac{9(-69-20 \sqrt{5})}{31375 \pi^{2}}$} & \multirow[t]{2}{*}{$\mp \frac{i \sqrt{5}}{25 \pi}$} \\
\hline & & & $-\frac{i \sqrt{5}}{10 \pi}$ & $\frac{i \sqrt{5}}{50 \pi}$ & & \\
\hline \multirow{4}{*}{$\frac{125}{2}+\frac{55}{2} \sqrt{5}$} & \multirow[t]{2}{*}{$\pm \frac{i(5 \sqrt{5}+11)}{4 \pi}$} & \multirow[t]{2}{*}{$\pm \frac{i 5(5 \sqrt{5}+11)}{4 \pi}$} & $\frac{i(5 \sqrt{5}+11)}{4 \pi}$ & $\frac{i 5(5 \sqrt{5}+11)}{4 \pi}$ & \multirow[t]{2}{*}{100.010534} & \multirow[t]{2}{*}{$\pm \frac{i 2(5 \sqrt{5}+11)}{4 \pi}$} \\
\hline & & & $-\frac{i(5 \sqrt{5}+11)}{4 \pi}$ & $-\frac{i 5(5 \sqrt{5}+11)}{4 \pi}$ & & \\
\hline & \multirow[t]{2}{*}{$\pm \frac{i(5 \sqrt{5}+11)}{4 \pi}$} & \multirow[t]{2}{*}{$\pm \frac{i 5(5 \sqrt{5}+11)}{4 \pi}$} & $\frac{i(5 \sqrt{5}+11)}{4 \pi}$ & $-\frac{i 5(5 \sqrt{5}+11)}{4 \pi}$ & \multirow[t]{2}{*}{38.200625} & \multirow[t]{2}{*}{$\pm \frac{i 2(5 \sqrt{5}+11)}{4 \pi}$} \\
\hline & & & $-\frac{i(5 \sqrt{5}+11)}{4 \pi}$ & $\frac{i 5(5 \sqrt{5}+11)}{4 \pi}$ & & \\
\hline \multirow{4}{*}{$-\frac{123}{2}+\frac{55}{2} \sqrt{5}$} & \multirow[t]{2}{*}{$\pm \frac{i \sqrt{5}}{50 \pi}$} & \multirow[t]{2}{*}{$\pm \frac{i \sqrt{5}}{50 \pi}$} & $\frac{i \sqrt{5}}{10 \pi}$ & $\frac{i \sqrt{5}}{10 \pi}$ & \multirow[t]{2}{*}{-0.000843} & \multirow[t]{2}{*}{$\pm \frac{i \sqrt{5}}{25 \pi}$} \\
\hline & & & $-\frac{i \sqrt{5}}{10 \pi}$ & $-\frac{i \sqrt{5}}{10 \pi}$ & & \\
\hline & \multirow[t]{2}{*}{$\pm \frac{i \sqrt{5}}{50 \pi}$} & $\pm \frac{i \sqrt{5}}{50 \pi}$ & $\frac{i \sqrt{5}}{10 \pi}$ & $-\frac{i \sqrt{5}}{10 \pi}$ & -0.000674 & $\mp \frac{i \sqrt{5}}{25 \pi}$ \\
\hline & & & $-\frac{i \sqrt{5}}{10 \pi}$ & $\frac{i \sqrt{5}}{10 \pi}$ & & \\
\hline & $\pm \frac{i \sqrt{5}}{50 \pi}$ & $\pm \frac{i \sqrt{5}}{10 \pi}$ & $\frac{i \sqrt{5}}{50 \pi}$ & $\frac{i \sqrt{5}}{10 \pi}$ & -0.001278 & $\mp \frac{i \sqrt{5}}{25 \pi}$ \\
\hline$\frac{125}{2}-\frac{55}{2} \sqrt{5}$ & & & $-\frac{i \sqrt{5}}{50 \pi}$ & $-\frac{i \sqrt{5}}{10 \pi}$ & & \\
\hline & $\pm \frac{i \sqrt{5}}{50 \pi}$ & $\pm \frac{i \sqrt{5}}{10 \pi}$ & $\frac{i \sqrt{5}}{50 \pi}$ & $-\frac{i \sqrt{5}}{10 \pi}$ & -0.003346 & $\pm \frac{i \sqrt{5}}{25 \pi}$ \\
\hline & & & $-\frac{i \sqrt{5}}{50 \pi}$ & $\frac{i \sqrt{5}}{10 \pi}$ & & \\
\hline & $\pm \frac{i(5 \sqrt{5}+11)}{4 \pi}$ & $\pm \frac{i(5 \sqrt{5}+11)}{4 \pi}$ & $\frac{i 5(5 \sqrt{5}+11)}{4 \pi}$ & $\frac{i 5(5 \sqrt{5}+11)}{4 \pi}$ & 303.899917 & $\pm \frac{i 2(5 \sqrt{5}+11)}{4 \pi}$ \\
\hline$-\frac{123}{2}-\frac{55}{2} \sqrt{5}$ & & & $-\frac{i 5(5 \sqrt{5}+11)}{4 \pi}$ & $-\frac{i 5(5 \sqrt{5}+11)}{4 \pi}$ & & \\
\hline & $\pm \frac{i(5 \sqrt{5}+11)}{4 \pi}$ & $\pm \frac{i(5 \sqrt{5}+11)}{4 \pi}$ & $\frac{i 5(5 \sqrt{5}+11)}{4 \pi}$ & $-\frac{i 5(5 \sqrt{5}+11)}{4 \pi}$ & -10.195921 & $\pm \frac{i 2(5 \sqrt{5}+11)}{4 \pi}$ \\
\hline & & & $-\frac{i 5(5 \sqrt{5}+11)}{4 \pi}$ & $\frac{i 5(5 \sqrt{5}+11)}{4 \pi}$ & & \\
\hline
\end{tabular}

Table 4. The parameters obtained from $\Gamma_{1}(5)$. Using (3.21), we can get the values for $\alpha_{i}$ 's and $Q$. 


\begin{tabular}{|c|c|c|c|c|c|c|}
\hline$\zeta=\mathrm{e}^{2 \pi \mathrm{i} \tau}$ & $m_{0}$ & $m_{1}$ & $m_{2}$ & $m_{3}$ & $U$ & $a$ \\
\hline \multirow{4}{*}{$\frac{1}{2}$} & \multirow[t]{2}{*}{$\pm \frac{i}{4 \pi}$} & \multirow[t]{2}{*}{$\pm \frac{i \sqrt{109}}{2 \pi}$} & $\frac{2 i}{\pi}$ & $\frac{27 i}{4 \pi}$ & \multirow[t]{2}{*}{$\frac{595}{48 \pi^{2}}$} & \multirow[t]{2}{*}{ $\pm 0.30258 i$} \\
\hline & & & $-\frac{2 i}{\pi}$ & $-\frac{27 i}{4 \pi}$ & & \\
\hline & \multirow[t]{2}{*}{$\pm \frac{i}{4 \pi}$} & \multirow[t]{2}{*}{$\pm \frac{i \sqrt{109}}{2 \pi}$} & $\frac{2 i}{\pi}$ & $-\frac{27 i}{4 \pi}$ & \multirow[t]{2}{*}{$-\frac{269}{48 \pi^{2}}$} & \multirow[t]{2}{*}{ $\pm 0.30258 i$} \\
\hline & & & $-\frac{2 i}{\pi}$ & $\frac{27 i}{4 \pi}$ & & \\
\hline \multirow{4}{*}{$\frac{1}{2}$} & \multirow[t]{2}{*}{$\pm \frac{i}{4 \pi}$} & \multirow[t]{2}{*}{$\pm \frac{2 i}{\pi}$} & $\frac{i \sqrt{109}}{2 \pi}$ & $\frac{27 i}{4 \pi}$ & \multirow[t]{2}{*}{$\frac{-665+108 \sqrt{109}}{48 \pi^{2}}$} & \multirow[t]{2}{*}{ $\pm 0.741431 i$} \\
\hline & & & $-\frac{i \sqrt{109}}{2 \pi}$ & $-\frac{2 i}{\pi}$ & & \\
\hline & \multirow[t]{2}{*}{$\pm \frac{i}{4 \pi}$} & \multirow[t]{2}{*}{$\pm \frac{2 i}{\pi}$} & $\frac{i \sqrt{109}}{2 \pi}$ & $-\frac{27 i}{4 \pi}$ & \multirow[t]{2}{*}{$\frac{-665-108 \sqrt{109}}{48 \pi^{2}}$} & \multirow[t]{2}{*}{ $\pm 0.741431 i$} \\
\hline & & & $-\frac{i \sqrt{109}}{2 \pi}$ & $\frac{27 i}{4 \pi}$ & & \\
\hline \multirow{4}{*}{2} & \multirow[t]{2}{*}{$\pm \frac{i}{2 \pi}$} & \multirow[t]{2}{*}{$\pm \frac{i \sqrt{109}}{\pi}$} & $\frac{27 i}{2 \pi}$ & $\frac{4 i}{\pi}$ & \multirow[t]{2}{*}{$\frac{455}{3 \pi^{2}}$} & \multirow[t]{2}{*}{ $\pm 0.6051525 i$} \\
\hline & & & $-\frac{27 i}{2 \pi}$ & $-\frac{4 i}{\pi}$ & & \\
\hline & \multirow[t]{2}{*}{$\pm \frac{i}{2 \pi}$} & \multirow[t]{2}{*}{$\pm \frac{i \sqrt{109}}{\pi}$} & $\frac{27 i}{2 \pi}$ & $-\frac{4 i}{\pi}$ & \multirow[t]{2}{*}{$\frac{23}{3 \pi^{2}}$} & \multirow[t]{2}{*}{ $\pm 0.6051525 i$} \\
\hline & & & $-\frac{27 i}{2 \pi}$ & $\frac{4 i}{\pi}$ & & \\
\hline \multirow{4}{*}{2} & \multirow[t]{2}{*}{$\pm \frac{i}{2 \pi}$} & \multirow[t]{2}{*}{$\pm \frac{4 i}{\pi}$} & $\frac{27 i}{2 \pi}$ & $\frac{i \sqrt{109}}{\pi}$ & \multirow[t]{2}{*}{$\frac{125+54 \sqrt{109}}{3 \pi^{2}}$} & \multirow[t]{2}{*}{ $\pm 1.4828632 i$} \\
\hline & & & $-\frac{27 i}{2 \pi}$ & $-\frac{i \sqrt{109}}{\pi}$ & & \\
\hline & \multirow[t]{2}{*}{$\pm \frac{i}{2 \pi}$} & \multirow[t]{2}{*}{$\pm \frac{4 i}{\pi}$} & $\frac{27 i}{2 \pi}$ & $-\frac{i \sqrt{109}}{\pi}$ & \multirow[t]{2}{*}{$\frac{125-54 \sqrt{109}}{3 \pi^{2}}$} & \multirow[t]{2}{*}{ $\pm 1.4828632 i$} \\
\hline & & & $-\frac{27 i}{2 \pi}$ & $\frac{i \sqrt{109}}{\pi}$ & & \\
\hline
\end{tabular}

Table 5. The parameters obtained from $\Gamma_{0}(6)$. Using (3.21), we can get the values for $\alpha_{i}$ 's and $Q$.

\begin{tabular}{|c|c|c|c|c|c|c|}
\hline$\zeta=\mathrm{e}^{2 \pi \mathrm{i} \tau}$ & $m_{0}$ & $m_{1}$ & $m_{2}$ & $m_{3}$ & $U$ & $a$ \\
\hline \multirow{4}{*}{$\frac{1}{2}$} & \multirow[t]{2}{*}{$\pm \frac{i}{16 \pi}$} & \multirow[t]{2}{*}{$\pm \frac{i}{8 \pi}$} & $\frac{i}{2 \pi}$ & $\frac{i}{16 \pi}$ & \multirow[t]{2}{*}{$\frac{11}{768 \pi^{2}}$} & \multirow[t]{2}{*}{ \pm 0.0528623} \\
\hline & & & $-\frac{i}{2 \pi}$ & $-\frac{i}{16 \pi}$ & & \\
\hline & \multirow[t]{2}{*}{$\pm \frac{i}{16 \pi}$} & \multirow[t]{2}{*}{$\pm \frac{i}{8 \pi}$} & $\frac{i}{2 \pi}$ & $-\frac{i}{16 \pi}$ & \multirow[t]{2}{*}{$-\frac{7}{256 \pi^{2}}$} & \multirow[t]{2}{*}{ \pm 0.0528623} \\
\hline & & & $-\frac{i}{2 \pi}$ & $\frac{i}{16 \pi}$ & & \\
\hline \multirow{4}{*}{2} & \multirow[t]{2}{*}{$\pm \frac{i}{8 \pi}$} & \multirow[t]{2}{*}{$\pm \frac{i}{4 \pi}$} & $\frac{i}{8 \pi}$ & $-\frac{i}{\pi}$ & \multirow[t]{2}{*}{$\frac{7}{48 \pi^{2}}$} & \multirow[t]{2}{*}{ \pm 0.1057} \\
\hline & & & $-\frac{i}{8 \pi}$ & $\frac{i}{\pi}$ & & \\
\hline & \multirow[t]{2}{*}{$\pm \frac{i}{8 \pi}$} & \multirow[t]{2}{*}{$\pm \frac{i}{4 \pi}$} & $\frac{i}{8 \pi}$ & $\frac{i}{\pi}$ & \multirow[t]{2}{*}{$\frac{23}{48 \pi^{2}}$} & \multirow[t]{2}{*}{ \pm 0.1057} \\
\hline & & & $-\frac{i}{8 \pi}$ & $-\frac{i}{\pi}$ & & \\
\hline
\end{tabular}

Table 6. The parameters obtained from $\Gamma_{0}(8)$. Using (3.21), we can get the values for $\alpha_{i}$ 's and $Q$. 


\begin{tabular}{|c|c|c|c|c|c|c|}
\hline$\zeta=\mathrm{e}^{2 \pi \mathrm{i} \tau}$ & $m_{0}$ & $m_{1}$ & $m_{2}$ & $m_{3}$ & $U$ & $a$ \\
\hline \multirow{4}{*}{$\frac{1-i \sqrt{3}}{2}$} & \multirow[t]{2}{*}{$\pm \frac{i}{6 \sqrt{3} \pi}$} & \multirow[t]{2}{*}{$\pm \frac{i}{6 \sqrt{3} \pi}$} & $\frac{i}{6 \sqrt{3} \pi}$ & $\frac{i \sqrt{3}}{2 \pi}$ & \multirow[t]{2}{*}{$-\frac{i(33 i+25 \sqrt{3})}{162 \pi^{2}}$} & \multirow[t]{2}{*}{$\pm(-0.1402495+0.0315441 i)$} \\
\hline & & & $-\frac{i}{6 \sqrt{3} \pi}$ & $-\frac{i \sqrt{3}}{2 \pi}$ & & \\
\hline & \multirow[t]{2}{*}{$\pm \frac{i}{6 \sqrt{3} \pi}$} & \multirow[t]{2}{*}{$\pm \frac{i}{6 \sqrt{3} \pi}$} & $\frac{i}{6 \sqrt{3} \pi}$ & $-\frac{i \sqrt{3}}{2 \pi}$ & \multirow[t]{2}{*}{$-\frac{i(3 i+8 \sqrt{3})}{81 \pi^{2}}$} & \multirow[t]{2}{*}{$\pm(-0.0887502+0.0362071 i)$} \\
\hline & & & $-\frac{i}{6 \sqrt{3} \pi}$ & $\frac{i \sqrt{3}}{2 \pi}$ & & \\
\hline \multirow{4}{*}{$\frac{1+i \sqrt{3}}{2}$} & \multirow[t]{2}{*}{$\pm \frac{i}{6 \sqrt{3} \pi}$} & \multirow[t]{2}{*}{$\pm \frac{i}{6 \sqrt{3} \pi}$} & $\frac{i}{6 \sqrt{3} \pi}$ & $\frac{i \sqrt{3}}{2 \pi}$ & \multirow[t]{2}{*}{$\frac{i(-33 i+25 \sqrt{3})}{162 \pi^{2}}$} & \multirow[t]{2}{*}{$\pm(-0.1402495-0.0315441 i)$} \\
\hline & & & $-\frac{i}{6 \sqrt{3} \pi}$ & $-\frac{i \sqrt{3}}{2 \pi}$ & & \\
\hline & \multirow[t]{2}{*}{$\pm \frac{i}{6 \sqrt{3} \pi}$} & \multirow[t]{2}{*}{$\pm \frac{i}{6 \sqrt{3} \pi}$} & $\frac{i}{6 \sqrt{3} \pi}$ & $-\frac{i \sqrt{3}}{2 \pi}$ & \multirow[t]{2}{*}{$\frac{i(-3 i+8 \sqrt{3})}{81 \pi^{2}}$} & \multirow[t]{2}{*}{$\pm(-0.0887502-0.0362071 i)$} \\
\hline & & & $-\frac{i}{6 \sqrt{3} \pi}$ & $\frac{i \sqrt{3}}{2 \pi}$ & & \\
\hline
\end{tabular}

Table 7. The parameters obtained from $\Gamma_{0}(9)$. Using (3.21), we can get the values for $\alpha_{i}$ 's and $Q$.

\begin{tabular}{|c|c|}
\hline$\Gamma(3)$ & 0 \\
\hline$\Gamma_{0}(4) \cap \Gamma(2)$ & $\frac{35152}{9}$ \\
\hline$\Gamma_{1}(5)$ & $\frac{131072}{9}$ \\
\hline$\Gamma_{0}(6)$ & -3072 \\
\hline$\Gamma_{0}(8)$ & $\frac{21952}{9}$ \\
\hline$\Gamma_{0}(9)$ & 0 \\
\hline
\end{tabular}

Table 8. The $j$-invariants that correspond to the six index-12 Belyi maps.

\begin{tabular}{|c|c|c|c|}
\hline Dessin & $\zeta$ & $\zeta^{\prime}$ & $\zeta^{\prime \prime}$ \\
\hline$\Gamma(3)$ & $\frac{1}{2}(1 \pm i \sqrt{3})$ & $\frac{1}{2}(1 \mp i \sqrt{3})$ & $\frac{1}{2}(1 \mp i \sqrt{3})$ \\
\hline$\Gamma_{0}(4) \cap \Gamma(2)$ & 2 & $\frac{1}{2}$ & -1 \\
\hline$\Gamma_{1}(5)$ & $\frac{1}{2}-\frac{11}{50} \sqrt{5}$ & $\frac{125}{2}+\frac{55}{2} \sqrt{5}$ & $\frac{1}{2}+\frac{11}{50} \sqrt{5}$ \\
\cline { 2 - 4 } & $-\frac{123}{2}+\frac{55}{2} \sqrt{5}$ & $-\frac{123}{2}-\frac{55}{2} \sqrt{5}$ & $\frac{125}{2}-\frac{55}{2} \sqrt{5}$ \\
\hline$\Gamma_{0}(6)$ & 2 & $\frac{1}{2}$ & - \\
\hline$\Gamma_{0}(8)$ & 2 & $\frac{1}{2}$ & - \\
\hline$\Gamma_{0}(9)$ & $\frac{1}{2}(1 \pm i \sqrt{3})$ & - & $\frac{1}{2}(1 \mp i \sqrt{3})$ \\
\hline
\end{tabular}

Table 9. The parametrizations for each case are related by triality. The hyphens indicate that such $\zeta$ either gives no solution to mass parameters $\left(\Gamma_{0}(6)\right.$ and $\left.\Gamma_{0}(8)\right)$ or does not satisfy the transformations of masses $\left(\Gamma_{0}(9)\right)$. 


\subsection{Minimal models and $\Gamma(3)$}

As an example, let us match the parametrizations for $\Gamma(3)$ obtained above to 4-point $\mathrm{CBs}$ in minimal models. In fact, as we will see, such CB first appears for the tetracritical Ising model when $p^{\prime}=6$ and $p=5$, that is, $c=4 / 5$. As usual, we can write the 4 -point $\mathrm{CB}$ as

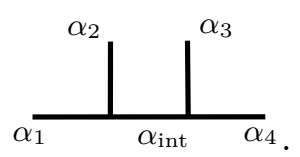

Then the intermediate field $\phi_{k, l}$ should satisfy the fusion rule

$$
\phi_{r, s} \times \phi_{m, n}=\sum_{\substack{k=|m-r|+1 \\ k-m+r-1 \in 2 \mathbb{Z}}}^{\min (m+r-1,2 p-1-m-r) \min \left(n+s-1,2 p^{\prime}-1-n-s\right)} \sum_{\substack{l=|n-s|+1 \\ l-n+s-1 \in 2 \mathbb{Z}}} \phi_{k, l},
$$

where the entire conformal family of a primary is implicit in the above abuse of notation. Let $\phi_{r_{i}, s_{i}}$ correspond to $\alpha_{1,4}$ and $\phi_{m_{i}, n_{i}}$ correspond to $\alpha_{2,3}(i=1,2)$. Then the fusion rule for the 4-point $\mathrm{CB}$ is

$$
\phi_{k, l} \in \phi_{r_{1}, s_{1}} \times \phi_{m_{1}, n_{1}}, \phi_{k, l} \in \phi_{r_{2}, s_{2}} \times \phi_{m_{2}, n_{2}}
$$

with constraints on $k, l$ indicated in (3.33).

Before we insert the specific values of the parametrizations, we can make some simplifications:

- Recall that the mass parameters are real or pure imaginary. If we have some parametrization with $m_{i} \in \mathbb{R}$, without loss of generality we can choose $\epsilon_{1}<0<\epsilon_{2}$. Then since $\frac{\epsilon_{1}+\epsilon_{2}}{\sqrt{\epsilon_{1} \epsilon_{2}}}=Q=\mathrm{i}\left(\sqrt{\frac{p^{\prime}}{p}}-\sqrt{\frac{p}{p^{\prime}}}\right)$, we have $\sqrt{\epsilon_{1} \epsilon_{2}}=-\mathrm{i} \epsilon_{2} \sqrt{\frac{p}{p^{\prime}}}$. Likewise, for some parametrization with $m_{i} \in \mathrm{i} \mathbb{R}$, without loss of generality we can choose $\epsilon_{1} / \mathrm{i}<0<\epsilon_{2} / \mathrm{i}$. Such two cases related by $m_{i} \rightarrow \mathrm{i} m_{i}$ should give the same $\epsilon_{1,2}$ up to a factor of $i$.

- If we make the choice in the above point for some specific $m_{i}$, then $m_{i} \rightarrow-m_{i}$ should give the same CFT parameters with $\epsilon_{1,2} \rightarrow \mathrm{i} \epsilon_{1,2}$. If we only have $m_{0} \rightarrow-m_{0}$ or $m_{1} \rightarrow-m_{1}$, then we should always get the same parametrization even without changing $\epsilon_{1,2}$ since the corresponding conformal dimension is $\frac{Q^{2}}{4}-\frac{m_{0,1}^{2}}{\epsilon_{1} \epsilon_{2}}$.

- Swapping $m_{2} \leftrightarrow m_{3}$ and swapping $m_{0} \leftrightarrow m_{1}$ simultaneously should give the same CFT parameters (for same $\epsilon_{1,2}$ ) due to the AGT map. This simply corresponds to read the $\mathrm{CB}$ (3.32) from the left or from the right.

In light of these points, it suffices to only contemplate one parametrization, ${ }^{8}$ say $m_{0}=$ $-m_{1}=m_{2}=-m_{3}=-\frac{1}{2 \sqrt{3}}$, for $\Gamma(3)$. When $p^{\prime}=6, p=5$, we find that there is only one possibility for $\Delta_{1}$ and $\Delta_{4}$, that is,

$$
\Delta_{1}=\Delta_{4}=\frac{1}{15}
$$

\footnotetext{
${ }^{8}$ Since $\Delta_{1}=\Delta_{4}$, when considering $\zeta \leftrightarrow 1 / \zeta$, it is equivalent to swapping both $m_{2} \leftrightarrow m_{3}$ and $m_{0} \leftrightarrow m_{1}$. Therefore, $\zeta=(1 \pm i \sqrt{3}) / 2$ should give the same parametrizations. Even if $|\zeta| \neq 1$, as long as $\Delta_{1}=\Delta_{4}$, swapping $2 \leftrightarrow 3$ always gives same CFT parameters as the extra factor of $1 /|\zeta|$ can be absorbed into $\sqrt{\epsilon_{1} \epsilon_{2}}$.
} 


\begin{tabular}{|c|cccccccc|}
\hline$\Delta_{1}$ & $(2,3)$ & $(2,3)$ & $(2,3)$ & $(2,3)$ & $(3,3)$ & $(3,3)$ & $(3,3)$ & $(3,3)$ \\
$\Delta_{2}$ & $(2,2)$ & $(2,2)$ & $(3,4)$ & $(3,4)$ & $(2,2)$ & $(2,2)$ & $(3,4)$ & $(3,4)$ \\
$\Delta_{3}$ & $(1,2)$ & $(4,4)$ & $(1,2)$ & $(4,4)$ & $(1,2)$ & $(4,4)$ & $(1,2)$ & $(4,4)$ \\
$\Delta_{4}$ & $(3,3)$ & $(2,3)$ & $(2,3)$ & $(3,3)$ & $(2,3)$ & $(3,3)$ & $(3,3)$ & $(2,3)$ \\
$\Delta_{\text {int }}$ & $(3,4)$ & $(3,4)$ & $(2,2)$ & $(2,2)$ & $(2,2)$ & $(2,2)$ & $(3,4)$ & $(3,4)$ \\
\hline
\end{tabular}

Table 10. There are 8 possible combinations. Each column gives a CB. In the leftmost column, $\Delta_{i}$ 's follow the nomenclature correpsonding to (3.36). For (3.37), it just swaps $2 \leftrightarrow 3$ (and $\Delta_{1}=\Delta_{4}$ ). Therefore, it essentially gives the same CBs. In other words, the two solutions just correspond to reading the 4-point $\mathrm{CB}$ (3.32) from left or from right.

There are two possible solutions for the remaining mass parameters (and deformation parameters):

$$
\begin{aligned}
& \epsilon_{2}=\frac{2}{\sqrt{3} \pi}, \quad \Delta_{2}=\frac{1}{40}, \quad \Delta_{3}=\frac{1}{8} \\
& \epsilon_{2}=-\frac{2}{\sqrt{3} \pi}, \quad \Delta_{2}=\frac{1}{8}, \quad \Delta_{3}=\frac{1}{40} .
\end{aligned}
$$

Moreover, for the intermediate channel,

$$
a=-\frac{1}{3 \sqrt{3} \pi}, \Delta_{\text {int }}=\frac{1}{40} .
$$

Hence, the intermediate channel $(k, l)$ obtained from $\Gamma(3)$ corresponds to $(2,2)$ or $(3,4)$ (and another $(k, l)$ satifying the fusion rule but not from the dessin is $(2,4)$ or $(3,2)$ ). It is not hard to see that the above two solutions both give the $8 \mathrm{CBs}$ in table 10 .

In fact, this corresponds to not only a CB in the tetracritical Ising model, but also CBs in many other minimal models. In figure 3, we give the Kac tables for a few examples.

By looking at these examples, one might see some patterns of the minimal models and the positions of conformal dimensions in cyan appeared in the Kac tables. Now, we are going to show

Proposition 3.2. The dessin $\Gamma(3)$ gives rise to the charges/momenta of the states in 4-point conformal blocks, where the corresponding weights of the primaries satisfy the conditions in table 11, in minimal models.

Following the specific values for $m_{i}$ and $a$, we can define $M_{0}:=\frac{m_{0}}{\sqrt{\epsilon_{1} \epsilon_{2}}}$ so that

$$
\alpha_{1}=-M_{0}+\frac{Q}{2}, \alpha_{2}=-M_{0}, \alpha_{3}=M_{0}, \alpha_{4}=M_{0}+\frac{Q}{2}, \alpha_{\text {int }}=\frac{2 M_{0}}{3}+\frac{Q}{2} .
$$

There are two possible choices for $\Delta_{1}$ in the Kac table. For future convenience, let us denote them as $\Delta_{r_{1}, s_{1}}$ and $\Delta_{r_{2}, s_{2}}$. Then

$$
\frac{\left(p^{\prime} r_{i}-p s_{i}\right)^{2}-\left(p^{\prime}-p\right)^{2}}{4 p^{\prime} p}=\frac{Q^{2}}{4}-M_{0}^{2}=-\frac{\left(p^{\prime}-p\right)^{2}}{4 p^{\prime} p}-M_{0}^{2} .
$$

Therefore,

$$
M_{0}^{2}=-\frac{\left(p^{\prime} r_{i}-p s_{i}\right)^{2}}{4 p^{\prime} p}
$$




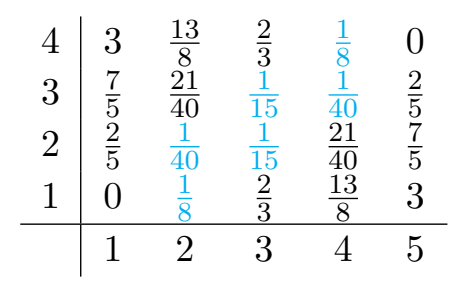

(a)

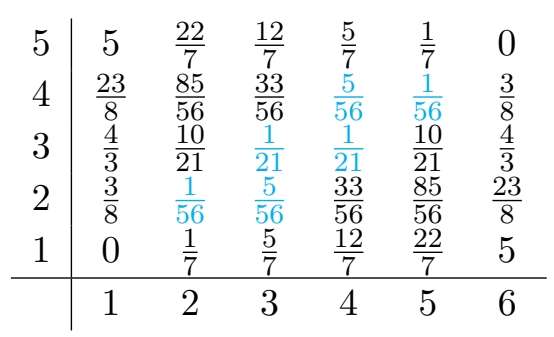

(c)

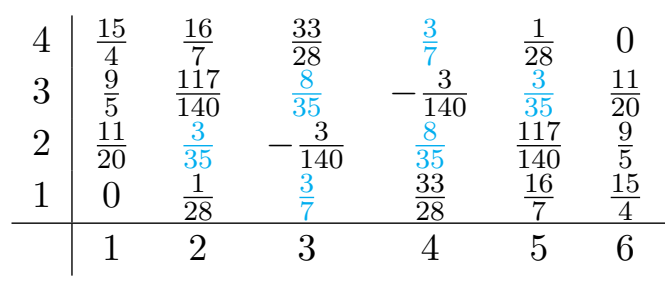

(b)

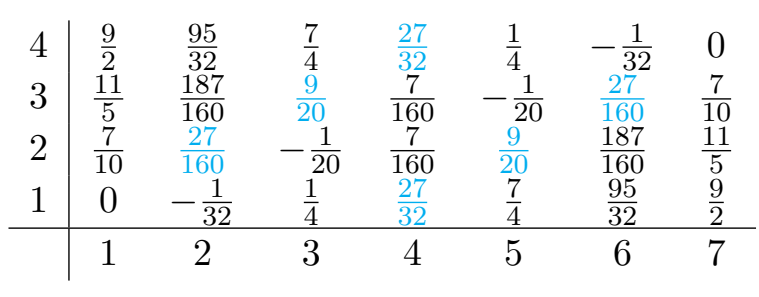

(d)

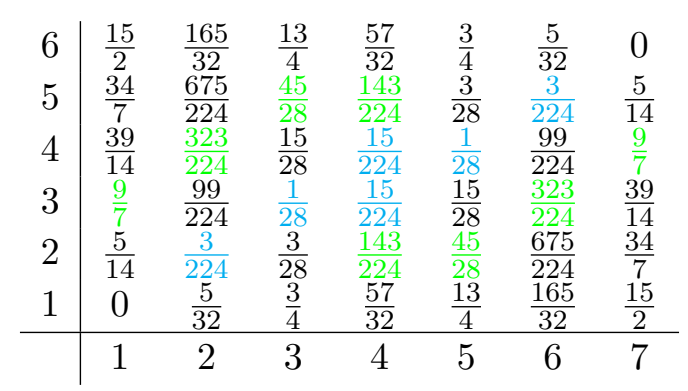

(e)

Figure 3. Here we list the first five possible examples of CBs that $\Gamma(3)$ corresponds to: (a) $p^{\prime}=6, p=5$, (b) $p^{\prime}=7, p=5$, (c) $p^{\prime}=7, p=6$, (d) $p^{\prime}=8, p=5$, (e) $p^{\prime}=8, p=7$. Those appeared in the CBs are in cyan in the Kac tables. For (e), we also have another combination of CBs in green.

It is also immediate from (3.39) that $\Delta_{1}=\Delta_{4}$. Hence, we can denote $\Delta_{2}$ or 3 as $\Delta_{m_{i}, n_{i}}$ without specifying whether $\left(m_{1,2}, n_{1,2}\right)$ corresponds to $\Delta_{2}$ or $\Delta_{3}$. We can plug this into $\Delta_{m_{i}, n_{i}}=\Delta_{3}=-M_{0}^{2}+Q M_{0}$ and get

$$
\left(p^{\prime} m_{i}-p^{\prime} n_{i}+x n_{2}\right)^{2}-x^{2}=\left(p^{\prime} r_{j}-p^{\prime} s_{j}+x s_{j}\right)^{2}-2 x\left(p^{\prime} r_{j}-p^{\prime} s_{j}+x s_{j}\right),
$$

where $x:=p^{\prime}-p$ is some positive integer. Its expansion gives

$$
\begin{aligned}
& p^{\prime 2}\left(m_{i}-n_{i}\right)^{2}+2 p^{\prime}\left(m_{i}-n_{i}\right) x n_{i}+x^{2} n_{i}^{2}-x^{2} \\
& =p^{\prime 2}\left(r_{j}-s_{j}\right)^{2}+2 p^{\prime}\left(r_{j}-s_{j}\right)\left(s_{j}-1\right) x+x^{2} s_{j}^{2}-2 x^{2} s_{j} .
\end{aligned}
$$

Since this is for general $p^{\prime}$, by comparing coefficients at different orders of $p^{\prime}$, we have

$$
m_{i}-n_{i}= \pm\left(r_{j}-s_{j}\right), n_{i}= \pm\left(s_{j}-1\right), n_{i}^{2}-1=s_{j}^{2}-2 s_{j},
$$




\begin{tabular}{|c|c|}
\hline Cases & Conditions \\
\hline All & $\left(r_{1}, s_{1}\right)=\left(p-r_{2}, p^{\prime}-s_{2}\right) \in 3(\mathbb{Z}, \mathbb{Z})$ \\
\hline$\left(r_{1}, s_{1}\right),\left(r_{1}+1, s_{1}+1\right),\left(r_{1}-1, s_{1}-1\right),\left(r_{1}, s_{1}\right)$ & \multirow{4}{*}{$\begin{array}{c}r_{1} \leq \frac{3(p-1)}{4}, s_{1} \leq \frac{3\left(p^{\prime}-1\right)}{4} \\
k=\frac{2}{3} r_{1}, l=\frac{2}{3} s_{1}\end{array}$} \\
\hline$\left(r_{1}, s_{1}\right),\left(r_{1}+1, s_{1}+1\right),\left(r_{2}+1, s_{2}+1\right),\left(r_{2}, s_{2}\right)$ & \\
\hline$\left(r_{1}, s_{1}\right),\left(r_{1}-1, s_{1}-1\right),\left(r_{2}-1, s_{2}-1\right),\left(r_{2}, s_{2}\right)$ & \\
\hline$\left(r_{2}, s_{2}\right),\left(r_{2}+1, s_{2}+1\right),\left(r_{2}-1, s_{2}-1\right),\left(r_{2}, s_{2}\right)$ & \\
\hline$\left(r_{2}, s_{2}\right),\left(r_{1}+1, s_{1}+1\right),\left(r_{1}-1, s_{1}-1\right),\left(r_{2}, s_{2}\right)$ & \multirow{4}{*}{$\begin{array}{c}\left(\frac{p+1}{2} \leq r_{1} \leq p-2\right. \\
\left.\text { or } \frac{p+1}{2} \leq r_{1} \leq \frac{3(p-1)}{4} \text { or } p=2 r_{1}\right) \\
\text { and }\left(\text { similar relations }{ }^{(*)} \text { with } p \rightarrow p^{\prime}, r_{1} \rightarrow s_{1}\right) \\
\text { and } k=p-\frac{2}{3} r_{1}, l=p^{\prime}-\frac{2}{3} s_{1}\end{array}$} \\
\hline$\left(r_{2}, s_{2}\right),\left(r_{1}+1, s_{1}+1\right),\left(r_{2}+1, s_{2}+1\right),\left(r_{1}, s_{1}\right)$ & \\
\hline$\left(r_{2}, s_{2}\right),\left(r_{1}-1, s_{1}-1\right),\left(r_{2}-1, s_{2}-1\right),\left(r_{1}, s_{1}\right)$ & \\
\hline$\left(r_{1}, s_{1}\right),\left(r_{2}+1, s_{2}+1\right),\left(r_{2}-1, s_{2}-1\right),\left(r_{1}, s_{1}\right)$ & \\
\hline
\end{tabular}

$(*)$ : note that the relation with $p=2 r_{1}$ and $p^{\prime}=2 s_{1}$ is automatically ruled out as $p^{\prime}-p=1$.

Table 11. The possible CBs of minimal models that $\Gamma(3)$ corresponds to.

where \pm can be seen from the symmetry of $p^{\prime 2}$ and $p^{\prime}$ terms in (3.43). Due to a similar symmetry for $\left(m_{i}, n_{i}\right) \leftrightarrow\left(p-m_{i}, p^{\prime}-n_{i}\right)$, it is possible to replace $\left(m_{i}, n_{i}\right)$ with $\left(p-m_{i}, p^{\prime}-n_{i}\right)$ or $\left(r_{j}, s_{j}\right)$ with $\left(p-r_{j}, p^{\prime}-s_{j}\right)$ in (3.42). It turns out that they also give the same set of equations. The third equation is actually redundant, and hence we have

$$
m_{i}-n_{i}= \pm\left(r_{j}-s_{j}\right), n_{i}= \pm\left(s_{j}-1\right) .
$$

Strictly speaking, in (3.42), we should really have $\left|p^{\prime} r_{j}-p^{\prime} s_{j}+x s_{j}\right|$ on the right hand side. Taking this into account, we would obtain another set of solutions with -1 replaced by +1 . Therefore,

$$
\begin{aligned}
m_{i} & =r_{j}-1, n_{i}=s_{j}-1, \\
\text { or } \quad m_{i} & =r_{j}+1, n_{i}=s_{j}+1 .
\end{aligned}
$$

As we also have similar relations for $\Delta_{2}$ and we have seen that $\Delta_{m_{1}, n_{1}} \neq \Delta_{m_{2}, n_{2}}$ for $Q \neq 0$, we learn that

$$
\left(m_{i}, n_{i}\right)=\left(r_{j}, s_{j}\right) \pm(1,1),\left(m_{1}, n_{1}\right) \neq\left(m_{2}, n_{2}\right), \quad\left(m_{1}, n_{1}\right) \neq\left(p-m_{2}, p^{\prime}-n_{2}\right) .
$$

For the intermediate channel, using $\frac{a}{\sqrt{\epsilon_{1} \epsilon_{2}}}=\frac{2 M_{0}}{3}$, we have

$$
\left(p^{\prime} k-p^{\prime} l+l\right)^{2}=\frac{4}{9}\left(p^{\prime} r_{j}-p^{\prime} s_{j}+s_{j}\right)^{2},
$$

so likewise,

$$
\begin{aligned}
k & =\frac{2}{3} r_{1}, & l & =\frac{2}{3} s_{1}, \\
\text { or } k & =p-\frac{2}{3} r_{1}, & l & =p^{\prime}-\frac{2}{3} s_{1},
\end{aligned}
$$

where without loss of generality we have chosen $j=1$ for convenience. As $k, l$ are integers, we must have $r_{1}, s_{1} \in 3 \mathbb{Z}$ (or in other words, $\left(p-r_{2}\right),\left(p^{\prime}-s_{2}\right) \in 3 \mathbb{Z}$ ). As $p=p^{\prime}-1$, 
it is straightforward to see that $k, l \in 2 \mathbb{Z}$ for $(3.50)$ while $(k, l) \in(2 \mathbb{Z}, 2 \mathbb{Z}+1)$ or $(k, l) \in$ $(2 \mathbb{Z}+1,2 \mathbb{Z})$ for $(3.51)$.

We also need to take the fusion rule into account. In general, there are $2^{2} \times\left(\begin{array}{l}4 \\ 2\end{array}\right)=24$ possible choices of external legs, where $2^{2}$ is the number of choices of $\Delta_{1}$ and $\Delta_{4}$ and $\left(\begin{array}{l}4 \\ 2\end{array}\right)$ corresponds to the choices of $\Delta_{2} \neq \Delta_{3}$. Therefore, we can discuss these possibilities case by case. Here, we will provide the details for three representative cases as examples. ${ }^{9}$

Example 1: $r_{1}, r_{1}+1, r_{1}-1, r_{1}$. In such case, the fusion rule gives

$$
\begin{aligned}
& 2 \leq k \leq \min \left(2 r_{1}, 2 p-2 r_{1}-2\right) \\
& 2 \leq k \leq \min \left(2 r_{1}-2,2 p-2 r_{1}\right) .
\end{aligned}
$$

Putting them together, we have

$$
2 \leq k \leq \min \left(2 r_{1}-2,2 p-2 r_{1}-2\right)
$$

Therefore,

$$
r_{1} \geq 2, p-r_{1} \geq 2 \text {. }
$$

In fact, we can omit $r_{1} \geq 2$ as we already have $r_{1} \in 3 \mathbb{Z}$. Furthermore, we also require $k-\left(r_{1}+1\right)+r_{1}-1 \in 2 \mathbb{Z}$, that is, $k \in 2 \mathbb{Z}$. We can write similar conditions for $l$. In particular, $l$ should also be even, so $(k, l)$ should obey (3.50). Therefore, we also need to plug (3.50) into the above inequality. This gives

$$
r_{1} \leq \frac{3(p-1)}{4}, s_{1} \leq \frac{3\left(p^{\prime}-1\right)}{4} .
$$

Comparing $p-2$ with $3(p-1) / 4$, we find that $p-2 \leq 3(p-1) / 4$ only when $p \leq 5$ (with equality at $p=5)$. However, for $p \leq 4$, we cannot have $p-r_{1} \geq 2$ as $r_{1} \in 3 \mathbb{Z}$. Hence, $r_{1} \leq \min (p-2,3(p-1) / 4)=3(p-1) / 4$ and likewise for $s_{1}$. In all, the conditions for this case are

$$
r_{1} \leq \frac{3(p-1)}{4}, s_{1} \leq \frac{3\left(p^{\prime}-1\right)}{4}, k=\frac{2}{3} r_{1}, l=\frac{2}{3} s_{1} .
$$

Example 2: $r_{\mathbf{2}}, r_{1}+\mathbf{1}, r_{1}-\mathbf{1}, r_{2}$. In such case, it is not hard to see that $k$ and $l$ should satisfy (3.51). Besides, the fusion rule gives

$$
\begin{aligned}
& \left|p-2 r_{1}-1\right|+1 \leq k \leq p-2 ; \\
& \left|p-2 r_{1}+1\right|+1 \leq k \leq p-2 .
\end{aligned}
$$

Putting them together, we have

$$
\max \left(\left|p-2 r_{1}-1\right|+1,\left|p-2 r_{1}+1\right|+1\right) \leq k \leq p-2 .
$$

Since $p-2 r_{1}-1<p-2 r_{1}+1$, there are three possibilities:

\footnotetext{
${ }^{9}$ Below we will use the correpsonding $r$ 's for external legs to denote each case.
} 
1. $p-2 r_{1}-1 \geq 0$ : if

$$
p \geq 2 r_{1}+1
$$

then

$$
p-2 r_{1}+2 \leq k \leq p-2 .
$$

Plugging $k=p-\frac{2}{3} r_{1}$ into (3.60), one may check that (3.59) and (3.60) are indeed consistent (they give the conditions $r_{1} \geq 2 / 3$ and $r_{1} \geq 3$ which are automatic as $\left.r_{1} \in 3 \mathbb{Z}\right)$.

2. $p-2 r_{1}+1 \leq 0$ : if

$$
p \leq 2 r_{1}-1
$$

then

$$
2 r_{1}-p+2 \leq k \leq p-2 .
$$

For this inequality to hold, we need $p \geq r_{1}+2$. Plugging $k=p-\frac{2}{3} r_{1}$ into the inequalities, we need $r_{1} \leq \frac{3(p-1)}{4}$. Following the above same reasoning, it suffices to keep $r_{1} \leq \frac{3(p-1)}{4}$.

3. $p-2 r_{1}=0:$ if

$$
p=2 r_{1}
$$

then

$$
2 \leq k \leq p-2 .
$$

Plugging $k=p-\frac{2}{3} r_{1}$ into the inequalities, one may check that these inequalities are indeed consistent (they give the conditions $r_{1} \geq 2 / 3$ and $r_{1} \geq 3$ which are automatic as $\left.r_{1} \in 3 \mathbb{Z}\right)$.

The disussion for $p^{\prime}, l, s_{1}$ is the same.

Example 3: $r_{1}, r_{1}+1, r_{1}-1, r_{2}$ In such case, the fusion rule gives

$$
\begin{aligned}
& 2 \leq k \leq \min \left(2 r_{1}, 2 p-2 r_{1}-2\right) \\
& 2 \leq k \leq \min \left(r_{1}+r_{2}-2,2 p-r_{1}-r_{2}\right)=\min (p-2, p)=p-2 .
\end{aligned}
$$

Putting them together, we have

$$
2 \leq k \leq \min \left(2 r_{1}-2,2 p-2 r_{1}-2, p-2\right) .
$$

Therefore,

$$
r_{1} \geq 2, p \geq 4, p-r_{1} \geq 2,
$$

where we can omit the first two conditions as we already have $r_{1} \in 3 \mathbb{Z}$. Furthermore, we also require $k-\left(r_{1}+1\right)+r_{1}-1 \in 2 \mathbb{Z}$, that is, $k \in 2 \mathbb{Z}$. We can write the similar conditions for $l$. In particular, $l$ should also be even. However, we also have $k-\left(r_{1}-1\right)+r_{2}-1 \in 2 \mathbb{Z}$, that is, $k-r_{1}-r_{2}=k-p=k-p^{\prime}+1 \in 2 \mathbb{Z}$. Likewise, $l-p^{\prime} \in 2 \mathbb{Z}$. This means that $k, l$ cannot be even at the same time (i.e., they should satisfy (3.51)). Hence, we reach an contradiction and this case is not possible. 
In fact, we can still reduce the number of cases to be checked. Since $r_{1}=p-r_{2}$, we have $r_{1} \pm 1=p-\left(r_{2} \mp 1\right)$. Therefore, we can rule out the cases where we choose $r_{1} \pm 1, r_{2} \mp 1$ from the $\left(\begin{array}{l}4 \\ 2\end{array}\right)$ possibilities as $\Delta_{2} \neq \Delta_{3}$. Hence, there are 16 cases (including the above three examples) overall. Moreover, just like in Example 3, we see that it fails to satisfy the fusion rule due to the parity of $k, l$. This can also be used to reduce the number of possible cases. One may check that

$$
\begin{aligned}
& r=r_{i}, m=r_{j} \pm 1, i=j \Rightarrow(k, l) \in 2(\mathbb{Z}, \mathbb{Z}) ; \\
& r=r_{i}, m=r_{j} \pm 1, i \neq j \Rightarrow(k, l) \in(2 \mathbb{Z}, 2 \mathbb{Z}+1) \text { or }(2 \mathbb{Z}+1,2 \mathbb{Z}) .
\end{aligned}
$$

This further reduces the number of possible cases (including the first two examples) to 8 . Although there are 8 distinct cases, there are only two conditions as in Example 1 and 2. This is because for the combination $r_{i}, r_{i} \pm 1, r_{j} \pm 1, r_{j}$, we always have

$$
2 \leq k \leq \min \left(2 r_{1}-2,2 p-2 r_{1}-2\right),
$$

and for the combination $r_{i}, r_{j \neq i} \pm 1, r_{\iota}, r_{\kappa \neq \iota} \pm 1$, we always have

$$
\max \left(\left|p-2 r_{1}-1\right|+1,\left|p-2 r_{1}+1\right|+1\right) \leq k \leq p-2 .
$$

This completes the proof, and the above conditions are summarized in table 11 . We can also see why the tetracritical Ising model is the one with smallest $p^{\prime}$ for $\Gamma(3)$. One way is to compute $p^{\prime}=3,4,5$ (with possible $p$ ) case by case, and none of them would give parametrizations from $\Gamma(3)$. Alternatively, it is straightforward to use the above conditions as well. Likewise, we can deduce that the smallest possible $p$ is 5 . Moreover, this also tells us why we cannot have $r_{1}=6$ or $s_{1}=6$ for $p^{\prime}=6,7$ and why $s_{1}=6$ is not allowed for $\left(p^{\prime}, p\right)=(8,5)$ as in figure 3 etc.

If a minimal model has CBs corresponding to $\Gamma(3)$, then $\left(r_{1}, s_{1}\right)=(3,3)$ (and hence $\left.\left(r_{2}, s_{2}\right)=\left(p-3, p^{\prime}-3\right)\right)$ must be one solution. It is not hard to find that $(k, l)$ is $(2,2)$ or $\left(p-2, p^{\prime}-2\right)$, and either $\Delta_{2}$ or $\Delta_{3}$ corresponds to $(2,2)$ or $\left(p-2, p^{\prime}-2\right)$ for all the eight cases. Therefore, we may use this to solve $M_{0}$ and $\epsilon_{1,2}$. Suppose $\Delta_{\text {int }}=\Delta_{3}$, then

$$
\frac{Q^{2}}{4}-\frac{4}{9} M_{0}^{2}=-M_{0}^{2}+Q M_{0}
$$

Hence, $M_{0}=\frac{3 Q}{10}$ or $\frac{3 Q}{2}$ with $Q=\frac{i}{\sqrt{p^{\prime}\left(p^{\prime}-1\right)}}$. If we consider $\Delta_{\text {int }}=\Delta_{2}$ (which we have seen that this would give no new $\mathrm{CBs}$ ), then we have the opposite values, that is, $M_{0}=-\frac{3 Q}{10}$ or $-\frac{3 Q}{2}$. Using $M_{0}=\frac{m_{0}}{\sqrt{\epsilon_{1} \epsilon_{2}}}$ and $\sqrt{\epsilon_{1} \epsilon_{2}} Q=\epsilon_{1}+\epsilon_{2}$, we may also solve $\epsilon_{1,2}$.

\subsection{Minimal models and $\Gamma_{0}(4) \cap \Gamma(2)$}

Let us now discuss one more example, $\Gamma_{0}(4) \cap \Gamma(2)$. We first focus on the cases when $\zeta=1 / 2$. In terms of the simplifications we can make as above, there are only two cases we need to consider. Again, we set $M_{0}=\frac{m_{0}}{\sqrt{\epsilon_{1} \epsilon_{2}}}$. In particular, one can find that the two cases only differ by $\Delta_{3}$. However, after some calculations, the fusion rule would always lead to $p^{\prime}, p \in 2 \mathbb{Z}$, which is impossible for coprime $p^{\prime}$ and $p$. 


\begin{tabular}{|c|c|}
\hline Cases & Conditions \\
\hline $2 r_{0}, 2 r_{0} \pm 1, r_{0} \pm 1, r_{0}$ & \multirow{4}{*}{$\begin{array}{c}r_{0} \leq \frac{p-1 \pm 1}{3} \\
k=2 r_{0}\end{array}$} \\
\hline $2 r_{0}, 2 r_{0} \pm 1, p-\left(r_{0} \pm 1\right), p-r_{0}$ & \\
\hline$p-2 r_{0}, p-\left(2 r_{0} \pm 1\right), r_{0} \pm 1, r_{0}$ & \\
\hline$p-2 r_{0}, p-\left(2 r_{0} \pm 1\right), p-\left(r_{0} \pm 1\right), p-r_{0}$ & \\
\hline$p-2 r_{0}, 2 r_{0} \pm 1, p-\left(r_{0} \pm 1\right), r_{0}$ & \multirow{4}{*}{$\begin{array}{c}\left(r_{0}<\frac{p \mp 1}{4} \text { or }\right. \\
\left.\frac{p \mp 1}{4} \leq r_{0} \leq \frac{p-1 / 2 \mp 1 / 2}{3} \text { or } r_{0}=\frac{p-1}{2}\right) \\
\text { and } k=p-2 r_{0}\end{array}$} \\
\hline$p-2 r_{0}, 2 r_{0} \pm 1, r_{0} \pm 1, p-r_{0}$ & \\
\hline $2 r_{0}, p-\left(2 r_{0} \pm 1\right), p-\left(r_{0} \pm 1\right), r_{0}$ & \\
\hline $2 r_{0}, p-\left(2 r_{0} \pm 1\right), r_{0} \pm 1, p-r_{0}$ & \\
\hline
\end{tabular}

Table 12. One set of possible CBs in minimal models that $\Gamma_{0}(4) \cap \Gamma(2)$ corresponds to. There are similar relations for $s_{0}, l, p^{\prime}$ by a simple substitution of the corresponding letters, where we have set $\alpha_{4}=\alpha_{r_{0}, s_{0}}$.

\begin{tabular}{|c|c|}
\hline Cases & Conditions \\
\hline $2 r_{0}, 2 r_{0} \pm 1, r_{0} \mp 1, r_{0}$ & $r_{0} \leq \frac{p-1 \pm 1}{3}$ \\
$k=2 r_{0}$ \\
\cline { 1 - 1 }$, 2 r_{0} \pm 1, p-\left(r_{0} \mp 1\right), p-r_{0}$ & \\
\hline$p-2 r_{0}, p-\left(2 r_{0} \pm 1\right), r_{0} \mp 1, r_{0}$ & $\left(r_{0}<\frac{p \mp 1}{4}\right.$ or \\
\cline { 1 - 1 }$-2 r_{0}, p-\left(2 r_{0} \pm 1\right), p-\left(r_{0} \mp 1\right), p-r_{0}$ & $\frac{p \mp 1}{4} \leq r_{0} \leq \frac{p-1 / 2 \mp 1 / 2}{3}$ or $\left.r_{0}=\frac{p-1}{2}\right)$ \\
\cline { 1 - 1 }$-2 r_{0}, 2 r_{0} \pm 1, p-\left(r_{0} \mp 1\right), r_{0}$ & and $k=p-2 r_{0}$ \\
\hline$p-2 r_{0}, 2 r_{0} \pm 1, r_{0} \mp 1, p-r_{0}$ & \\
\hline $2 r_{0}, p-\left(2 r_{0} \pm 1\right), p-\left(r_{0} \mp 1\right), r_{0}$ & \\
\hline $2 r_{0}, p-\left(2 r_{0} \pm 1\right), r_{0} \mp 1, p-r_{0}$ & \\
\hline
\end{tabular}

Table 13. The other set of possible CBs in minimal models that $\Gamma_{0}(4) \cap \Gamma(2)$ corresponds to. There are similar relations for $s_{0}, l, p^{\prime}$ by a simple substitution of the corresponding letters, where we have set $\alpha_{4}=\alpha_{r_{0}, s_{0}}$.

Next, for $\zeta=2$, it is very similar to $\zeta=1 / 2$ but with a swap of $m_{2}, m_{3}$ and an overall rescaling. We also have two distinct cases. For $(+,+,-,-),{ }^{10}$ using the same method yields the CBs in minimal models with conditions in table 12. Likewise, the other case with $(-,+,-,+)$ gives the conditions in table 13. It is not hard to see that for $(+,+,-,-)$, the first $\mathrm{CB}$ appears in the minimal model with $p^{\prime}=5, p=4$, viz, the tricritical Ising model. For $(-,+,-,+)$, the first CB appears in the minimal model with $p^{\prime}=4, p=3$, viz, the (critical) Ising model. The Kac tables and corresponding CBs are shown in figure 4.

Finally, let us consider $\zeta=-1$. Since $a$ always vanishes, $\Delta_{\text {int }}=\frac{Q^{2}}{4}=-\frac{\left(p^{\prime}-p\right)^{2}}{4 p^{\prime} p}$. Hence, $p^{\prime} k-p l=0$, that is, $p^{\prime} / p=l / k$. However, as $\operatorname{gcd}\left(p^{\prime}, p\right)=1$ and $k<p, l<p^{\prime}$, this is impossible.

Now that we have found two dessins that corresponds to CBs in minimal models, we can consider their CBs in the same minimal model. Such example would first appear when

\footnotetext{
${ }^{10}$ Here, it is still sufficient to choose two representatives for the two distinct cases. As different parametrizations of the masses would only differ by signs of $m_{i}$ 's, we will only use their signs to denote $\left(m_{0}, m_{1}, m_{2}, m_{3}\right)$. This should be clear from the tables in section 3.3.
} 

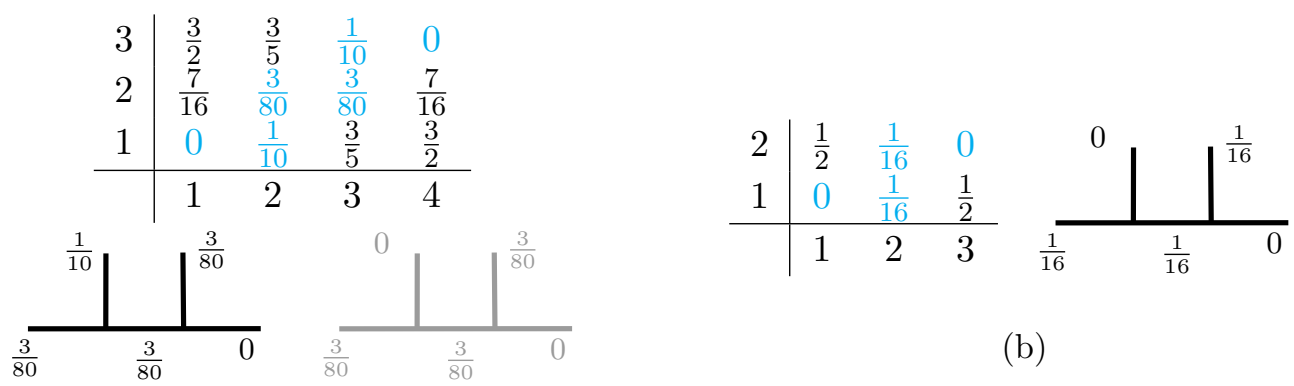

(b)

(a)

Figure 4. Here we list the first possible examples of CBs that $\Gamma_{0}(4) \cap \Gamma(2)$ corresponds to: (a) The first $\mathrm{CB}$ for $(+,+,-,-)$. For reference, the one in grey is the $\mathrm{CB}$ from $(-,+,-,+)$ for this minimal model. (b) The first CB for $(-,+,-,+)$.
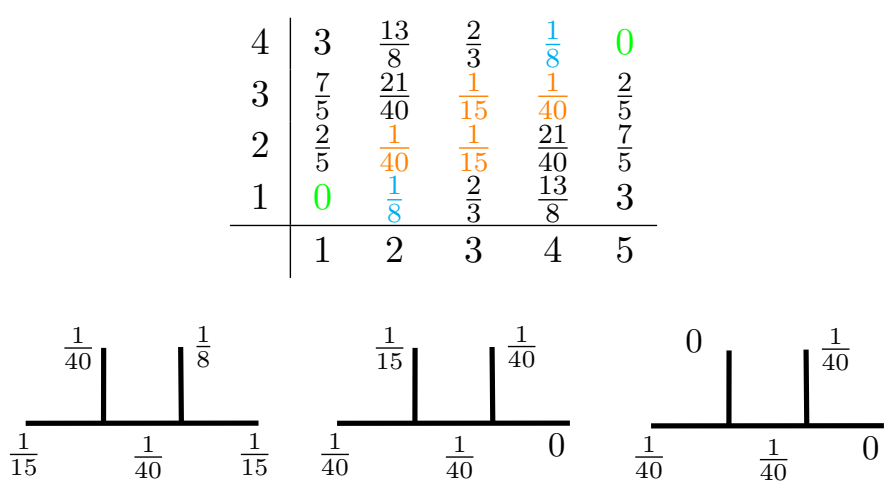

Figure 5. The CBs from $\Gamma(3)$ (cyan) and $\Gamma_{0}(4) \cap \Gamma(2)$ (green) in the tetracritical Ising model. The ones in orange appear for both of the dessins. The three CBs, from left to right, come from $\Gamma(3)$, $(+,+,-,-)$ and $(-,+,-,+)$ in $\Gamma_{0}(4) \cap \Gamma(2)$ respectively.

$p^{\prime}=6, p=5$ as in figure 5.

\subsection{Minimal models and general dessins}

Following the above steps, we can derive the results for any dessin in general.

Proposition 3.3. Suppose for a dessin, we have the gauge theory parameters with relation

$$
m_{1}= \pm / \mp k_{1} m_{0}, m_{2}= \pm / \mp k_{2} m_{0}, m_{3}= \pm / \mp k_{3} m_{0}, a= \pm / \mp k_{\mathrm{int}} m_{0},
$$

where $k_{i, \text { int }}>0$. Then the dessin corresponds to the states of 4-point CBs satisfying conditions in table 14 in minimal models.

In fact, we may further make the following conjecture.

Conjecture 3.4. For a dessin satisfying the conditions in proposition 3.3, it corresponds to a family of 4-point CBs whose states follow table 14.

So far, we have already discussed how a dessin can reproduce the charges/momenta of the states in a 4-point CB of a minimal model. However, as $\zeta$ is fixed for each dessin and we 


\begin{tabular}{|c|c|}
\hline Cases & Conditions \\
\hline$k_{1} r_{0}, k_{2} r_{0} \pm 1, k_{3} r_{0} \pm / \mp 1, r_{0}$ & \multirow{4}{*}{$\begin{array}{c}\max (|\boldsymbol{\Delta} \pm 1|,|\Delta \pm 1|)+1 \\
\leq k_{\text {int }} r_{0} \leq \frac{1}{2} \min (\star-3,4 p-3-\star) \\
\quad \text { and } k=k_{\text {int }} r_{0}\end{array}$} \\
\hline$k_{1} r_{0}, k_{2} r_{0} \pm 1, p-\left(k_{3} r_{0} \pm / \mp 1\right), p-r_{0}$ & \\
\hline$p-k_{1} r_{0}, p-\left(k_{2} r_{0} \pm 1\right), k_{3} r_{0} \pm / \mp 1, r_{0}$ & \\
\hline$p-k_{1} r_{0}, p-\left(k_{2} r_{0} \pm 1\right), p-\left(k_{3} r_{0} \pm / \mp 1\right), p-r_{0}$ & \\
\hline$p-k_{1} r_{0}, k_{2} r_{0} \pm 1, p-\left(k_{3} r_{0} \pm / \mp 1\right), r_{0}$ & \multirow{4}{*}{$\begin{array}{c}\max \left(\left|\left(k_{1}+k_{2}\right) r_{2} \pm 1\right|,\left|\left(k_{3}+1\right) r_{2} \pm 1\right|\right)+1 \\
\leq k_{\mathrm{int}} r_{0} \leq \min (p-2-|\mathbf{\Delta}|, p-2-|\Delta|), \\
\text { and } k=p-k_{\mathrm{int}} r_{0}\end{array}$} \\
\hline$p-k_{1} r_{0}, k_{2} r_{0} \pm 1, k_{3} r_{0} \pm / \mp 1, p-r_{0}$ & \\
\hline$k_{1} r_{0}, p-\left(k_{2} r_{0} \pm 1\right), p-\left(k_{3} r_{0} \pm / \mp 1\right), r_{0}$ & \\
\hline$k_{1} r_{0}, p-\left(k_{2} r_{0} \pm 1\right), k_{3} r_{0} \mp 1, p-r_{0}$ & \\
\hline
\end{tabular}

Table 14. The set of possible CBs in minimal models that a general dessin corresponds to. There are similar relations for $s_{0}, l, p^{\prime}$ by a simple substitution of the corresponding letters, where we have set $\alpha_{4}=\alpha_{r_{0}, s_{0}}$ and $\boldsymbol{\Delta}=\left(k_{2}-k_{1}\right) r_{0}, \Delta=\left(1-k_{3}\right) r_{0}, \star=\left(-\left|k_{1}+k_{2}-k_{3}-1\right|+\sum_{i} k_{i}\right) r_{0}$. In particular, $k_{i, \text { int }} r_{0} \in \mathbb{N}^{*}$ is a necessary condition.

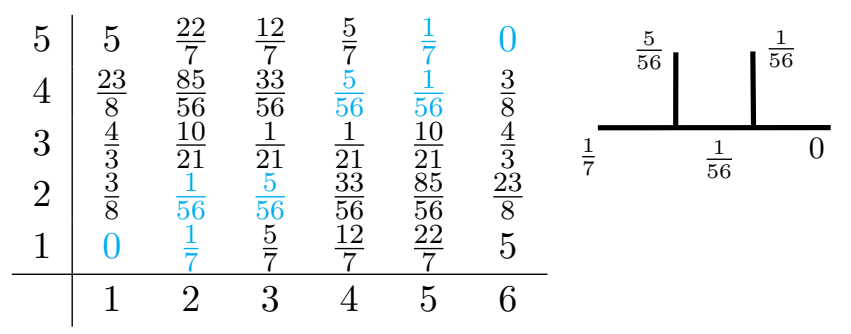

Figure 6. The $\mathrm{CB}$ on the right has conformal dimensions coloured cyan in the Kac table.

are only obtaining $\zeta$ by relating the Strebel and SW differentials rather than describing it as a concrete mathematical object in the language of dessins, further study on whether/how dessins could fully recover the CBs and the spectra is required.

With the conditions in table 14 , we can check what CBs in minimal models we can obtain from a dessin. For instance, when $\zeta=\frac{1}{2}+\frac{11}{50} \sqrt{5}$ for $\Gamma_{1}(5)$, we have $k_{2}=1, k_{1}=$ $k_{3}=5, k_{\text {int }}=2$. It is not hard to find that the first CB it corresponds to appears when $p^{\prime}=7, p=6$ as in figure 6 .

Examples not giving minimal models. From proposition 3.3, it is straightforward to see that there could be dessins that do not correspond to CBs in minimal models. Besides the inequalities in table 14 , a necessary condition is that $k_{i \text {,int }} r_{0}$ and $k_{i \text {,int }} s_{0}$ should be positive integers. Let us verify this with some examples.

For $\Gamma_{0}(6)$, there are two big classes of parametrizations. If $m_{2}$ or $m_{3}$ has the factor $\sqrt{109}$, then we cannot get the rational conformal dimensions for all the external legs. If instead $m_{1}$ has the factor $\sqrt{109}$, all the conformal dimensions can be rational since $\Delta_{1}=\frac{Q^{2}}{4}-M_{0}^{2}$. However, if we now express $M_{0}$ in terms of the labels $\left(r_{2}, s_{2}\right)$ for $\Delta_{4}$ and insert this into $\Delta_{1}$, we find that

$$
\left(p^{\prime} r_{1}-p s_{1}\right)^{2}=4 \times 109\left(p^{\prime} r_{2}-p s_{2}\right)^{2},
$$


where 109 is not a square number, and hence no integer solutions (except when $0=0$ which is excluded for minimal models). Therefore, it is not possible to get CBs in minimal models for $\Gamma_{0}(6)$.

For $\Gamma_{0}(8), m_{i}$ and $a$ are non-zero and cannot simultaneously be real/pure imaginary as in table 6 . Without loss of generality, suppose $\frac{m_{i}}{\sqrt{\epsilon_{1} \epsilon_{2}}}$ is pure imaginary and then $\frac{a}{\sqrt{\epsilon_{1} \epsilon_{2}}}$ is real. This yields

$$
\Delta_{\text {int }}=\frac{Q^{4}}{4}-\frac{a^{2}}{\epsilon_{1} \epsilon_{2}}<\frac{Q^{2}}{4} .
$$

Therefore,

$$
\frac{\left(p^{\prime} k-p l\right)^{2}-\left(p^{\prime}-p\right)^{2}}{4 p^{\prime} p}<\frac{Q^{2}}{4}=-\frac{\left(p^{\prime}-p\right)^{2}}{4 p^{\prime} p}
$$

In other words,

$$
\left(p^{\prime} k-p l\right)^{2}<0
$$

Hence, it is not possible to get CBs in minimal models for $\Gamma_{0}(8)$.

For $\Gamma_{0}(9)$, since the $a$ 's are not real or pure imaginary, it should not give CBs in minimal models.

\section{Acknowledgments}

OF wishes to thank R. Santachiara for useful discussions on the topic of this note, as well as related topics. The research of JB is supported by the CSC scholarship. OF is supported by the Australian Reasearch Council. YHH would like to thank STFC for grant ST/J00037X/1. EH would like to thank STFC for the PhD studentship. YX is supported by NSFC grant No. 20191301017. FY is supported by the NSFC grant No. 11950410490, by Fundamental Research Funds for the Central Universities A0920502051904-48, by Startup research grant A1920502051907-2-046, in part by NSFC grant No. 11501470 and No. 11671328, and by Recruiting Foreign Experts Program No. T2018050 granted by SAFEA.

\section{A The B-model and omega deformations}

When mapping gauge theory/SW geometry parameters to CFT parameters, we need to include a factor of $\frac{1}{\sqrt{\epsilon_{1} \epsilon_{2}}}$, which would lead to divergence under the flat space limit $\epsilon_{1,2} \rightarrow 0$. Here, we discuss a way in terms of topological B-model so that the SW geometry is still physically meaningful when $\epsilon_{1,2}$ are non-zero.

Recall that we have related $\mathcal{N}=2$ gauge theories to A-model topological strings. The mirror in B-model is defined by the equation

$$
v w+f(x, y)=0
$$

which is a $\mathrm{CY}_{3}$ that can be considered as fibration of $u v=c$ for some constant $c$ over the Riemann surface $f(x, y)$. In particular, $f(x, y)=0$ can be identified as the SW curve $\Sigma$. Denote the multiplicity of a BPS state in this $5 \mathrm{~d}$ theory as $N^{\beta}$, where $\beta$ is essentially the 
charge of the BPS state. ${ }^{11}$ Mathematically, the BPS configuration can be defined by a (complex) one-dimensional sheaf $\mathcal{F}$ (plus certain section in $H^{0}(\mathcal{F})$ ) such that

$$
\beta=\operatorname{ch}_{2}(\mathcal{F}), n=\chi(\mathcal{F})
$$

where $\beta \in H_{2}(\mathcal{M}, \mathbb{Z})$ and $n \in \mathbb{Z}$.

The topological string amplitude then has the expansion

$$
F\left(\epsilon_{1}, \epsilon_{2}, t\right)=\log (Z)=\sum_{n, g=0}^{\infty}\left(\epsilon_{1}+\epsilon_{2}\right)^{2 n}\left(\epsilon_{1} \epsilon_{2}\right)^{g-1} F^{(n, g)}(t),
$$

where $Z$ is known as the (refined) Pandharipande-Thomas (PT) partition function, and $g$ stands for the genus while $t$ denotes the Kähler parameter measuring the volume of a curve in $\beta$, which can be identified as the Coulomb parameter $a$ as we are focusing on $\operatorname{SU}(2)$ gauge group in this paper [50-52]. In particular, when $n=g=0, F^{(0,0)}$ is the prepotential $\mathcal{F}$. In the limit $\epsilon_{1,2} \rightarrow 0$, the PT partition function is naturally identified as the Nekrasov partition function at leading order:

$$
\log (Z)=\left(\epsilon_{1} \epsilon_{2}\right)^{-1} F^{(0,0)} .
$$

Moreover, $F^{(0,1)}$ and $F^{(1,0)}$ can also be determined using the metric on $\mathcal{M}$ and the discriminant of $\Sigma$ as in Equation (3.22) and (3.23) in [52]. Then $F^{(n, g)}$ with higher $(g+n)$ can be deduced from the (generalized) holomorphic anomaly equation [50-52]

$$
\bar{\partial}_{\bar{i}} F^{(n, g)}=\frac{1}{2} \bar{C}_{\bar{i}}^{j k}\left(\mathrm{D}_{j} \mathrm{D}_{k} F^{(n, g-1)}+\sum_{m, h}^{\prime} \mathrm{D}_{j} F^{(m, h)} \mathrm{D}_{k} F^{(n-m, g-h)}\right), g+n>1,
$$

where the three-point coupling $\bar{C}_{\bar{i}}^{j k}$ is given in $[51,52]$, and $\mathrm{D}_{i}$ is the covariant derivative. The prime in the sum indicates the omission of $(m, h)=(0,0),(n, g)$. We also require the first term on the right hand side to vanish if $g=0$.

Therefore, the non-zero $\epsilon_{1,2}$ would also make sense for the SW theory physically as the prepotential generates the topological string amplitudes. Hence, we could avoid the divergence when mapping the gauge theory parameters to CFT parameters as in section 3 .

\section{B Brane configurations}

\section{B.1 The type IIA brane configuration}

A type IIA configuration of parallel NS/D5-branes joined by D4-branes can be represented in $\mathrm{M}$ theory as a single M5-brane with a more complicated world history.

Before we write the rule for finding the Seiberg-Witten curve, we need to find out whether we have a $\mathrm{U}(N)$ or an $\mathrm{SU}(N)$ gauge theory. This is discussed in [53], and goes as follows.

\footnotetext{
${ }^{11}$ More precisely, we should also include the indices denoting the $\mathrm{SU}(2)_{L} \times \mathrm{SU}(2)_{R}$ spin representations, but for our purpose here, it suffices to label it with the topological data $\beta$ only. For more details, see for example [50].
} 
First, consider D5-branes and D4-branes in type IIA superstring theory. The worldvolume of a D5-brane is described as follows. D5-branes are located at $x^{7}=x^{8}=x^{9}=0$ and, in a semi-classical approximation, at fixed values of $x^{6}$. The world-volume of D5-branes are parameterised by values of $x^{0}, x^{1}, \cdots, x^{5}$. In addition, D4-branes are parameterised by $x^{0}, x^{1}, x^{2}, x^{3}$ and $x^{6}$. D4-branes have their $x^{6}$-coordinate finite so that they terminate on D5-branes. We need to introduce a complex variable $v=x^{4}+i x^{5}$. Classically, every D4brane is located at a definite value of $v$. Since a D4-brane ending on a D5-brane creates a dimple in the D5-brane, the value $x^{6}$ is the value measured at $v=\infty$, far from the disturbance created by the D4-brane. By minimizing the volume of the D5-brane, at large $v$, we obtain

$$
x^{6}=k \ln |v|+\text { const. }
$$

This is not well-defined for large $v$. Nevertheless, with D4-branes attached to the left and to the right of the D5-brane, we have

$$
x^{6}=k \sum_{i=1}^{q_{L}} \ln \left|v-a_{i}\right|-k \sum_{j=1}^{q_{R}} \ln \left|v-b_{j}\right|+\text { const },
$$

where $a_{i}$ and $b_{j}$ are the $v$-values, or $x^{6}$-coordinates of D4-branes ending on the left and right respectively. Now $x^{6}$ is well-defined for large $v$ if and only if $q_{L}=q_{R}$, that is, if the forces on both sides are balanced. For infrared divergence, we need to consider the motion of the D4-branes, whose movement causes the D5-brane to move. The motion of a D5-brane contributes to the kinetic energy of the D4-brane. The D5-brane kinetic energy is given by $\int \mathrm{d}^{4} x \mathrm{~d}^{2} v \sum_{\mu=0}^{3} \partial_{\mu} x^{6} \partial^{\mu} x^{6}$. Therefore, with $x^{6}$ in (B.2), we have

$$
k^{2} \int \mathrm{d}^{4} x \mathrm{~d}^{2} v\left|\operatorname{Re}\left(\sum_{i} \frac{\partial_{\mu} a_{i}}{v-a_{i}}-\sum_{j} \frac{\partial_{\mu} b_{j}}{v-b_{j}}\right)\right|^{2} .
$$

This integral converges if and only if

$$
\partial_{\mu}\left(\sum_{i} a_{i}-\sum_{j} b_{j}\right)=0
$$

so that

$$
\sum_{i} a_{i}-\sum_{j} b_{j}=q_{\alpha}
$$

where $q_{\alpha}$ is characteristic of $\alpha$-th plane. From the D4-brane point of view, (B.5) means the $\mathrm{U}(1)$ part of $\mathrm{U}(k)$ for $k$ D4-branes between two D5-branes are frozen. This is because $\sum_{i} a_{i}$ is the scalar part of $\mathrm{U}(1)$ vector multiplet in one factor $\mathrm{U}\left(k_{\alpha}\right)$ and $\sum_{j} b_{j}$ is the scalar part of the $\mathrm{U}(1)$ vector multiplet in the factor $\mathrm{U}\left(k_{\alpha+1}\right)$. Since, following (B.5), the difference is fixed by supersymmetry, the entire $\mathrm{U}(1)$ vector multiplet is missing, and we have $\mathrm{SU}(N)$.

\section{B.2 The M-theory brane configuration}

The world-volume of the M5-brane is such that,

1. It has arbitrary values in the first $\mathbb{M}^{4}$ coordinates $x^{0}, \cdots, x^{3}$, and is located at $x^{7}=$ $x^{8}=x^{9}=0 ;$ 
2. In the remaining four coordinates, which parametrize a 4-manifold $Q \cong \mathbb{R}^{3} \times S^{1}$, D5-brane worldvolume spans a $2 \mathrm{~d}$ surface $\Sigma$;

3. The $\mathcal{N}=2$ supersymmetry means we give $Q$ the complex structure in which $v=$ $x^{4}+i x^{5}$ and $s=x^{6}+i x^{10}$ are holomorphic, then $\Sigma$ is a complex Riemann surface in $Q$. This makes $\mathbb{M}^{4} \times \Sigma$ a supersymmetric cycle in the sense of [54] and so it ensures spacetime supersymmetry.

When projected to type IIA brane diagrams, $\Sigma$ has different components described locally by saying that $s$ is constant (the D5-branes) or that $v$ is constant (the $\mathrm{D} 4$-branes). In type IIA, different components can meet and singularity appears in there. However, in going to $\mathrm{M}$ theory, singularities disappear. Hence, for generic values of parameters, $\Sigma$ will be a smooth Riemann surface in $Q$.

\section{Congruence subgroups of the modular group}

In this appendix, we very briefly recall some essential details regarding the modular group $\Gamma \equiv \Gamma(1)=\operatorname{PSL}(2, \mathbb{Z})=\mathrm{SL}(2, \mathbb{Z}) /\{ \pm I\}$, the group of linear fractional transformations $\mathbb{Z} \ni z \rightarrow \frac{a z+b}{c z+d}$, with $a, b, c, d \in \mathbb{Z}$ and $a d-b c=1$. It is generated by the transformations $T$ and $S$ defined by

$$
T(z)=z+1 \quad, \quad S(z)=-1 / z .
$$

The presentation of $\Gamma$ is $\left\langle S, T \mid S^{2}=(S T)^{3}=I\right\rangle$.

The most important subgroups of $\Gamma$ are the congruence subgroups, defined by having the entries in the generating matrices $S$ and $T$ obeying some modular arithmetic. Of particular note are the following:

- Principal congruence subgroups:

$$
\Gamma(m):=\left\{A \in \mathrm{SL}(2 ; \mathbb{Z}) ; A_{i j} \equiv \pm I_{i j} \bmod m\right\} /\{ \pm I\} ;
$$

- Congruence subgroups of level $m$ : subgroups of $\Gamma$ containing $\Gamma(m)$ but not any $\Gamma(n)$ for $n<m$;

- Unipotent matrices:

$$
\Gamma_{1}(m):=\left\{A \in \operatorname{SL}(2 ; \mathbb{Z}) ; A_{i j} \equiv \pm\left(\begin{array}{ll}
1 & b \\
0 & 1
\end{array}\right)_{i j} \bmod m\right\} /\{ \pm I\}
$$

- Upper triangular matrices:

$$
\Gamma_{0}(m):=\left\{\left(\begin{array}{ll}
a & b \\
c & d
\end{array}\right) \in \Gamma ; c \equiv 0 \bmod m\right\} /\{ \pm I\} .
$$

In $[6,48]$, attention is drawn to the conjugacy classes of a particular family of subgroups of $\Gamma$ : the so-called genus zero, torsion-free congruence subgroups: 
- Torsion-free means that the subgroup contains no element of finite order other than the identity.

- To explain genus zero, first recall that the modular group acts on the upper half-plane $\mathcal{H}:=\{\tau \in \mathbb{C}, \operatorname{Im}(\tau)>0\}$ by linear fractional transformations $z \rightarrow \frac{a z+b}{c z+d}$. Then $\mathcal{H}$ gives rise to a compactification $\mathcal{H}^{*}$ when adjoining cusps, which are points on $\mathbb{R} \sqcup \infty$ fixed under some parabolic element (i.e. an element $A \in \Gamma$ not equal to the identity and for which $\operatorname{Tr}(A)=2$ ). The quotient $\mathcal{H}^{*} / \Gamma$ is a compact Riemann surface of genus 0 , i.e. a sphere. It turns out that with the addition of appropriate cusp points, the extended upper half plane $\mathcal{H}^{*}$ factored by various congruence subgroups will also be compact Riemann surfaces, possibly of higher genus. Such a Riemann surface, as a complex algebraic variety, is called a modular curve. The genus of a subgroup of the modular group is the genus of the modular curve produced in this way.

The genus zero torsion-free congruence subgroups of the modular group are very rare: there are only 33 of them, with index $I \in\{6,12,24,36,48,60\}$, as detailed in [48].

\section{Elliptic curves and $j$-invariants}

Given the Weierstrass function $\wp$

$$
\wp\left(z \mid \omega_{1}, \omega_{2}\right)=\frac{1}{z^{2}}+\sum_{n^{2}+m^{2} \neq 0}\left(\frac{1}{\left(z+m \omega_{1}+n \omega_{2}\right)^{2}}-\frac{1}{\left(m \omega_{1}+n \omega_{2}\right)^{2}}\right),
$$

where $\omega_{1}$ and $\omega_{2}$ are complex-valued vectors that span the lattice $\Lambda=\left\{m \omega_{1}+n \omega_{2}\right.$ : $m, n \in \mathbb{Z}\}$, and we can write $\wp\left(z \mid \omega_{1}, \omega_{2}\right)=\wp(z \mid \Lambda)$. The embedding of a torus, as an elliptic curve over $\mathbb{C}$ in the complex projective plane, follows from

$$
\left(\wp^{\prime}(z)\right)^{2}=4(\wp(z))^{3}-g_{2} \wp(z)-g_{3},
$$

where $\wp^{\prime}(z)$ is the derivative of $\wp(z)$ with respect to $z$. Naturally defined on a torus $\mathbb{C} / \Lambda$, $\wp$ is doubly-periodic with respect to lattice $\Lambda$. This torus can be embedded in the complex projective plane by $z \mapsto\left[1: \wp(z): \wp^{\prime}(z)\right]$. Close to the origin, $\wp(z)$ can be expanded as

$$
\wp(z \mid \Lambda)=\frac{1}{z^{2}}+g_{2} \frac{z^{2}}{20}+g_{3} \frac{z^{4}}{28}+\mathcal{O}\left(z^{6}\right)
$$

where

$$
\begin{aligned}
& g_{2}=60 \sum_{(m, n) \neq(0,0)}\left(\frac{1}{m \omega_{1}+n \omega_{2}}\right)^{4}, \\
& g_{3}=140 \sum_{(m, n) \neq(0,0)}\left(\frac{1}{m \omega_{1}+n \omega_{2}}\right)^{6} .
\end{aligned}
$$

The summed terms in $g_{2}$ and $g_{3}$ are the first two Eisenstein series respectively. The Eisenstein series $G_{2 k}$ with weight $2 k$ are modular forms of weight $2 k$, that is, they transform 
as $G_{2 k}(\tau) \mapsto(c \tau+d)^{2 k} G_{2 k}(\tau)$ under $\operatorname{SL}(2, \mathbb{Z})$ with $\tau=\omega_{1} / \omega_{2}$ in upper half-plane $\mathcal{H}$. If two lattices are related by a multiplication by a non-zero complex number $c$, then the corresponding curves are isomorphic. The $j$-invariants are defined as

$$
j(\tau)=1728 \frac{g_{2}^{3}}{g_{2}^{3}-27 g_{3}^{2}} .
$$

This definition shows that the $j$-invariant is a weight-zero modular form. From the above discussion, we can see that each isomorphism class of elliptic curves over $\mathbb{C}$ has the same $j$-invariant.

As the SW curves and Strebel differentials we have are of quartic form, $y^{2}=a z^{4}+$ $b z^{3}+c z^{2}+d z+q^{2}$, we can make the substitution (for $q \neq 0$ )

$$
z=\frac{2 q(X+c)-d^{2} /(2 q)}{Y}, y=-q+\frac{1}{2 q} \frac{2 q(X+c)-d^{2} /(2 q)}{Y}\left(\frac{2 q(X+c)-d^{2} / 2 q}{Y}-d\right)
$$

so that the elliptic curve can be expressed in the standard Weierstrass form

$$
Y^{2}+a_{1} X Y+a_{3} Y=X^{3}+a_{2} X^{2}+a_{4} X+a_{6},
$$

where

$$
a_{1}=\frac{d}{q}, a_{2}=c-\frac{d^{2}}{4 q^{2}}, a_{3}=2 b q, a_{4}=-4 a q^{2}, a_{6}=a d^{2}-4 a c q^{2} .
$$

Using SAGE [55], we can compute its $j$-invariant

$$
j=-\frac{\left(\left(a_{1}^{2}+4 a_{2}\right)^{2}-24 a_{1} a_{3}-48 a_{4}\right)^{3}}{\left(a_{2} a_{3}^{2}-a_{1} a_{3} a_{4}+a_{1}^{2} a_{6}-a 4^{2}+4 a_{2} a_{6}\right)\left(a_{1}^{2}+4 a_{2}\right)^{2}+8\left(a_{1} a_{3}+2 a_{4}\right)^{3}-9\left(a_{1}^{2}+4 a_{2}\right)\left(a_{1} a_{3}+2 a_{4}\right)\left(a_{3}^{2}+4 a_{6}\right)+27\left(a_{3}^{2}+4 a_{6}\right)^{2}} .
$$

If $q=0$ such as the Strebel differential for $\Gamma(3)$, we can replace $z$ and $y$ with $1 / z$ and $y / z^{2}$ respectively to obtain a quartic form with a non-vanishing constant term [56].

\section{E Elliptic functions and Coulomb moduli}

\section{E.1 The elliptic integral of first kind}

We first give a quick review on deriving (3.6). From (3.1), we have

$$
\frac{\mathrm{d} \lambda_{\mathrm{SW}}}{\mathrm{d} U}=-\frac{1+\zeta}{2 \sqrt{P_{4}(z)}}
$$

Then

$$
\frac{\mathrm{d} a}{\mathrm{~d} U}=\frac{1}{2 \pi i} \oint_{A} \frac{\mathrm{d} \lambda_{\mathrm{SW}}}{\mathrm{d} U}=-\frac{2}{2 \pi i} \int_{\lambda_{1}}^{\lambda_{2}} \frac{1+\zeta}{2 \sqrt{P_{4}(z)}} \mathrm{d} z .
$$

Therefore, the integral boils down to solving

$$
\int_{\lambda_{1}}^{\lambda_{2}} \frac{\mathrm{d} z}{\sqrt{\left(z-\lambda_{1}\right)\left(z-\lambda_{2}\right)\left(z-\lambda_{3}\right)\left(z-\lambda_{4}\right)}} .
$$

First, we make a $\operatorname{PSL}(2, \mathbb{Z})$ transformation, $z=\frac{A t+B}{C t+D}$, such that $\lambda_{1,2,3}$ are mapped to $0,1, \infty$ respectively. Then $A=\left(\lambda_{1}-\lambda_{2}\right) \lambda_{3}, B=\left(\lambda_{2}-\lambda_{3}\right) \lambda_{1}, C=\lambda_{1}-\lambda_{2}, D=\lambda_{2}-\lambda_{3}$ 
gives a solution, and $\lambda_{4}=\frac{\left(\lambda_{2}-\lambda_{3}\right)\left(\lambda_{4}-\lambda_{1}\right)}{\left(\lambda_{1}-\lambda_{3}\right)\left(\lambda_{3}-\lambda_{4}\right)}=: t_{0}$. After substitution of variables and some algebra, the integral becomes

$$
\frac{1}{\sqrt{\left(\lambda_{2}-\lambda_{3}\right)\left(\lambda_{4}-\lambda_{1}\right)}} \int_{0}^{1} \frac{\mathrm{d} t}{\sqrt{t(1-t)\left(1-t_{0}^{-1} t\right)}} .
$$

In particular,

$$
\int_{0}^{1} \frac{\mathrm{d} t}{\sqrt{t(1-t)\left(1-t_{0}^{-1} t\right)}}=\pi_{2} F_{1}\left(\frac{1}{2}, \frac{1}{2} ; 1, t_{0}^{-1}\right)=2 K\left(t_{0}^{-1 / 2}\right),
$$

where $K$ is the elliptic integral of the first kind

$$
K(x)=\int_{0}^{1} \frac{\mathrm{d} t}{\sqrt{\left(1-t^{2}\right)\left(1-x^{2} t^{2}\right)}} .
$$

Hence,

$$
\int_{\lambda_{1}}^{\lambda_{2}} \frac{\mathrm{d} z}{\sqrt{\left(z-\lambda_{1}\right)\left(z-\lambda_{2}\right)\left(z-\lambda_{3}\right)\left(z-\lambda_{4}\right)}}=\frac{2 K\left(\sqrt{\frac{\left(\lambda_{1}-\lambda_{2}\right)\left(\lambda_{3}-\lambda_{4}\right)}{\left(\lambda_{2}-\lambda_{3}\right)\left(\lambda_{4}-\lambda_{1}\right)}}\right)}{\sqrt{\left(\lambda_{2}-\lambda_{3}\right)\left(\lambda_{4}-\lambda_{1}\right)}} .
$$

\section{E.2 The elliptic logarithm}

Here we present an alternative way to obtain $a$ from $\mathrm{d} a / \mathrm{d} U$ by integrating from $U$ to $\infty$. Therefore, we need to determine $a$ when $U \rightarrow \infty$. At large $U$, we have

$$
\left.\frac{P_{4}(z)}{U}\right|_{U \rightarrow \infty}=(-1-\zeta) z^{3}+(1+\zeta)^{2} z^{2}+(-\zeta(1+\zeta)) z
$$

which yields

$$
\left.P_{4}(z)\right|_{U \rightarrow \infty}=-(1+\zeta) U z^{3}+(1+\zeta)^{2} U z^{2}-\zeta(1+\zeta) U z .
$$

If $|\zeta| \leq 1$, then

$$
\begin{aligned}
\left.\frac{\mathrm{d} a}{\mathrm{~d} U}\right|_{U \rightarrow \infty} & =-\frac{1+\zeta}{2 \pi i} \int_{\lambda_{1}}^{\lambda_{2}}\left((-(1+\zeta) U)\left(z^{3}-(1+\zeta) z^{2}+\zeta z\right)\right)^{-1 / 2} \mathrm{~d} z \\
& =\frac{\sqrt{1+\zeta}}{2 \pi \sqrt{U}} \int_{1}^{\infty}\left(z^{3}-(1+\zeta) z^{2}+\zeta z\right)^{-1 / 2} \mathrm{~d} z \\
& =-\frac{\sqrt{1+\zeta}}{\pi \sqrt{U}} \operatorname{EL}(1,0 ;-1-\zeta, \zeta),
\end{aligned}
$$

where EL is the elliptic logarithm defined as

$$
\mathrm{EL}(x, y ; a, b)=\frac{1}{2} \int_{\infty}^{x} \frac{\mathrm{d} t}{\sqrt{t^{3}+a t^{2}+b t}}, \quad y=\sqrt{x^{3}+a x^{2}+b x} .
$$

Therefore,

$$
\left.a(U)\right|_{U \rightarrow \infty}=-\frac{2}{\pi} \sqrt{1+\zeta} \operatorname{EL}(1,0 ;-1-\zeta, \zeta) \sqrt{U}
$$


However, notice that the above steps are not rigorous. We need to be careful about the branches of square roots. Taking this into account, when $1+\zeta<0$, there should be an minus extra sign, ${ }^{12}$ that is,

$$
\left.a(U)\right|_{U \rightarrow \infty, \zeta<-1}=\frac{2}{\pi} \sqrt{1+\zeta} \operatorname{EL}(1,0 ;-1-\zeta, \zeta) \sqrt{U} .
$$

Henceforth, we will not repeat this point below. As a sanity check, we can see what would happen at weak coupling. When $\zeta=0, \operatorname{EL}(1,0 ;-1,0)=\pi / 2$. We learn that ${ }^{13}$

$$
\left.a(U)\right|_{U \rightarrow \infty, \zeta \rightarrow 0}=-\sqrt{U},
$$

which is the familiar behaviour in the (semi)classical limit.

If $|\zeta|>1$, then we can just replace $\int_{1}^{\infty}$ with $\int_{\zeta}^{\infty}$, and hence

$$
\left.a(U)\right|_{U \rightarrow \infty}=-\frac{2}{\pi} \sqrt{1+\zeta} \operatorname{EL}(\zeta, 0 ;-1-\zeta, \zeta) \sqrt{U} .
$$

Likewise, we can also write down a similar expression for $a_{D}$ at large $U$,

$$
a_{D}=-\frac{2}{\pi} \sqrt{1+\zeta} \operatorname{EL}(0,0 ;-1-\zeta, \zeta) \sqrt{U}
$$

If we take $\zeta=0$, then $\operatorname{EL}(0,0 ;-1,0)$ goes to $\infty$. This is expected as the monopoles are heavy for weak coupling.

Therefore, the integral for $a(U)$ can be written as

$$
a(U)=a(\infty)+\frac{1+\zeta}{m_{0} \pi \mathrm{i}} \int_{U}^{\infty} \frac{\mathrm{d} U^{\prime}}{\sqrt[4]{x_{2}\left(U^{\prime}\right)}} K\left(\frac{\sqrt[4]{x_{1}\left(U^{\prime}\right)}}{\sqrt[4]{x_{2}\left(U^{\prime}\right)}}\right)
$$

where $\int_{U}^{\infty}$, just like (3.12), could still be a sum of integrals due to the non-trivial monodromy.

Open Access. This article is distributed under the terms of the Creative Commons Attribution License (CC-BY 4.0), which permits any use, distribution and reproduction in any medium, provided the original author(s) and source are credited.

\section{References}

[1] L.F. Alday, D. Gaiotto and Y. Tachikawa, Liouville correlation functions from four-dimensional gauge theories, Lett. Math. Phys. 91 (2010) 167 [arXiv:0906.3219] [INSPIRE].

[2] N. Seiberg and E. Witten, Electric-magnetic duality, monopole condensation, and confinement in $N=2$ supersymmetric Yang-Mills theory, Nucl. Phys. B 426 (1994) 19 [Erratum ibid. 430 (1994) 485] [hep-th/9407087] [INSPIRE].

\footnotetext{
${ }^{12}$ In practice, we usually choose a large cutoff (which can be either positive or negative) for $U$ instead of $\infty$ when performing numerical integrals. Therefore, the branches of square roots with $U$ inside are also important if we take $U$ to a large negative number.

${ }^{13}$ We may also have an extra minus sign for all the expressions of $\left.a(U)\right|_{U \rightarrow \infty}$ here. Mathematically, this should correspond to choosing a different branch in the redefinition of square root. Physically, this is due to the action of Weyl group of the gauge symmetry.
} 
[3] N. Seiberg and E. Witten, Monopoles, duality and chiral symmetry breaking in $N=2$ supersymmetric QCD, Nucl. Phys. B 431 (1994) 484 [hep-th/9408099] [InSPIRE].

[4] N.A. Nekrasov, Seiberg-Witten prepotential from instanton counting, Adv. Theor. Math. Phys. 7 (2003) 831 [hep-th/0206161] [INSPIRE].

[5] D. Gaiotto, $N=2$ dualities, JHEP 08 (2012) 034 [arXiv:0904.2715] [INSPIRE].

[6] Y.-H. He and J. McKay, $N=2$ gauge theories: congruence subgroups, coset graphs and modular surfaces, J. Math. Phys. 54 (2013) 012301 [arXiv:1201.3633] [INSPIRE].

[7] Y.-H. He, J. McKay and J. Read, Modular subgroups, dessins d'enfants and elliptic K3 surfaces, J. Comp. Math. 16 (2013) 271 [arXiv:1211.1931] [INSPIRE].

[8] Y.-H. He and J. McKay, Eta products, BPS states and K3 surfaces, JHEP 01 (2014) 113 [arXiv: 1308.5233] [INSPIRE].

[9] Y.-H. He and J. Read, Dessins d'enfants in $\mathcal{N}=2$ generalised quiver theories, JHEP 08 (2015) 085 [arXiv : 1503.06418] [INSPIRE].

[10] Y.-H. He and J. McKay, Sporadic and exceptional, arXiv:1505.06742 [INSPIRE].

[11] S.K. Ashok, F. Cachazo and E. Dell'Aquila, Children's drawings from Seiberg-Witten curves, Commun. Num. Theor. Phys. 1 (2007) 237 [hep-th/0611082] [InSPIRE].

[12] Y.-H. He, E. Hirst and T. Peterken, Machine-learning dessins d'enfants: explorations via modular and Seiberg-Witten curves, J. Phys. A 54 (2021) 075401 [arXiv:2004.05218] [INSPIRE].

[13] T. Eguchi and K. Maruyoshi, Penner type matrix model and Seiberg-Witten theory, JHEP 02 (2010) 022 [arXiv: 0911.4797] [INSPIRE].

[14] C. Kozcaz, S. Pasquetti and N. Wyllard, A\& B model approaches to surface operators and Toda theories, JHEP 08 (2010) 042 [arXiv: 1004.2025] [INSPIRE].

[15] M. Bershtein and O. Foda, AGT, Burge pairs and minimal models, JHEP 06 (2014) 177 [arXiv: 1404.7075] [INSPIRE].

[16] K.B. Alkalaev and V.A. Belavin, Conformal blocks of $W_{N}$ minimal models and AGT correspondence, JHEP 07 (2014) 024 [arXiv:1404.7094] [INSPIRE].

[17] P. Degiovanni, Moore and Seiberg equations, topological theories and Galois theory, Helv. Phys. Acta 67 (1994) 799 [INSPIRE].

[18] D. Poland, S. Rychkov and A. Vichi, The conformal bootstrap: theory, numerical techniques, and applications, Rev. Mod. Phys. 91 (2019) 015002 [arXiv:1805.04405] [INSPIRE].

[19] A.A. Belavin, A.M. Polyakov and A.B. Zamolodchikov, Infinite conformal symmetry in two-dimensional quantum field theory, Nucl. Phys. B 241 (1984) 333 [INSPIRE].

[20] N. Nekrasov and A. Okounkov, Seiberg-Witten theory and random partitions, Prog. Math. 244 (2006) 525 [hep-th/0306238] [INSPIRE].

[21] R.J. Rodger, A pedagogical introduction to the AGT conjecture, Master's thesis, Utrecht University, Utrecht, The Netherlands (2013).

[22] I. Antoniadis, E. Gava, K.S. Narain and T.R. Taylor, Topological amplitudes in string theory, Nucl. Phys. B 413 (1994) 162 [hep-th/9307158] [INSPIRE]. 
[23] M. Bershadsky, S. Cecotti, H. Ooguri and C. Vafa, Kodaira-Spencer theory of gravity and exact results for quantum string amplitudes, Commun. Math. Phys. 165 (1994) 311 [hep-th/9309140] [INSPIRE].

[24] M. Aganagic, A. Klemm, M. Mariño and C. Vafa, The topological vertex, Commun. Math. Phys. 254 (2005) 425 [hep-th/0305132] [InSPIRE].

[25] A. Iqbal, C. Kozcaz and C. Vafa, The refined topological vertex, JHEP 10 (2009) 069 [hep-th/0701156] [INSPIRE].

[26] H. Awata and H. Kanno, Instanton counting, Macdonald functions and the moduli space of D-branes, JHEP 05 (2005) 039 [hep-th/0502061] [INSPIRE].

[27] H. Awata and H. Kanno, Refined BPS state counting from Nekrasov's formula and Macdonald functions, Int. J. Mod. Phys. A 24 (2009) 2253 [arXiv:0805.0191] [InSPIRE].

[28] M. Taki, Refined topological vertex and instanton counting, JHEP 03 (2008) 048 [arXiv:0710.1776] [INSPIRE].

[29] L. Bao, E. Pomoni, M. Taki and F. Yagi, M5-branes, toric diagrams and gauge theory duality, JHEP 04 (2012) 105 [arXiv: 1112.5228] [INSPIRE].

[30] L. Bao, V. Mitev, E. Pomoni, M. Taki and F. Yagi, Non-Lagrangian theories from brane junctions, JHEP 01 (2014) 175 [arXiv:1310.3841] [INSPIRE].

[31] O. Foda and J.-F. Wu, From topological strings to minimal models, JHEP 07 (2015) 136 [arXiv: 1504.01925] [INSPIRE].

[32] O. Foda and J.-F. Wu, A Macdonald refined topological vertex, J. Phys. A 50 (2017) 294003 [arXiv: 1701.08541] [INSPIRE].

[33] H. Hayashi, S.-S. Kim, K. Lee and F. Yagi, Equivalence of several descriptions for $6 d$ SCFT, JHEP 01 (2017) 093 [arXiv:1607.07786] [INSPIRE].

[34] S.-S. Kim, M. Taki and F. Yagi, Tao probing the end of the world, PTEP 2015 (2015) 083B02 [arXiv: 1504.03672] [INSPIRE].

[35] C. Hwang, J. Kim, S. Kim and J. Park, General instanton counting and 5d SCFT, JHEP 07 (2015) 063 [Addendum ibid. 04 (2016) 094] [arXiv:1406.6793] [INSPIRE].

[36] H. Hayashi, H.-C. Kim and T. Nishinaka, Topological strings and $5 d T_{N}$ partition functions, JHEP 06 (2014) 014 [arXiv:1310.3854] [INSPIRE].

[37] O. Bergman, D. Rodríguez-Gómez and G. Zafrir, Discrete $\theta$ and the $5 d$ superconformal index, JHEP 01 (2014) 079 [arXiv:1310.2150] [INSPIRE].

[38] O. Bergman, D. Rodríguez-Gómez and G. Zafrir, 5-brane webs, symmetry enhancement, and duality in 5d supersymmetric gauge theory, JHEP 03 (2014) 112 [arXiv:1311.4199] [INSPIRE].

[39] Y. Tachikawa, $N=2$ supersymmetric dynamics for pedestrians, Springer, Germany (2013) [arXiv: 1312.2684] [INSPIRE].

[40] N.C. Leung and C. Vafa, Branes and toric geometry, Adv. Theor. Math. Phys. 2 (1998) 91 [hep-th/9711013] [INSPIRE].

[41] A. Gorsky, S. Gukov and A. Mironov, SUSY field theories, integrable systems and their stringy/brane origin. 2., Nucl. Phys. B 518 (1998) 689 [hep-th/9710239] [INSPIRE]. 
[42] O. Aharony, A. Hanany and B. Kol, Webs of $(p, q)$ five-branes, five-dimensional field theories and grid diagrams, JHEP 01 (1998) 002 [hep-th/9710116] [INSPIRE].

[43] S.-S. Kim and F. Yagi, 5d $E_{n}$ Seiberg-Witten curve via toric-like diagram, JHEP 06 (2015) 082 [arXiv: 1411.7903] [INSPIRE].

[44] E. Girondo and G. González-Diez, Introduction to compact Riemann surfaces and dessins d'enfants, London Mathematical Society Student Texts, Cambridge University Press, Cambdirg U.K. (2011).

[45] G. Jones and D. Singerman, Belyi functions, hypermaps and Galois groups, Bull. London Math. Soc. 28 (1996) 561.

[46] A. Grothendieck, Esquisse d'un programme, (1984).

[47] M. Mulase and M. Penkava, Ribbon graphs, quadratic differentials on Riemann surfaces, and algebraic curves defined over $\bar{q}$, Asian J. Math. 2 (1998) 11.

[48] J. McKay and A. Sebbar, $j$-invariants of arithmetic semistable elliptic surfaces and graphs, CRM Proceedings and Lecture Notes, American Mathematical Society, U.S.A. (2001).

[49] P. Francesco, P. Mathieu and D. Sénéchal, Conformal field theory, Springer, Germany (2012).

[50] M.-X. Huang, A. Klemm and M. Poretschkin, Refined stable pair invariants for $E$-, $M$ - and [p,q]-strings, JHEP 11 (2013) 112 [arXiv:1308.0619] [INSPIRE].

[51] M.-x. Huang and A. Klemm, Direct integration for general $\Omega$ backgrounds, Adv. Theor. Math. Phys. 16 (2012) 805 [arXiv: 1009.1126] [INSPIRE].

[52] M.-x. Huang, A.-K. Kashani-Poor and A. Klemm, The $\Omega$ deformed B-model for rigid $\mathcal{N}=2$ theories, Annales Henri Poincaré 14 (2013) 425 [arXiv: 1109.5728] [INSPIRE].

[53] E. Witten, Solutions of four-dimensional field theories via M-theory, Nucl. Phys. B 500 (1997) 3 [hep-th/9703166] [INSPIRE].

[54] K. Becker, M. Becker and A. Strominger, Five-branes, membranes and nonperturbative string theory, Nucl. Phys. B 456 (1995) 130 [hep-th/9507158] [InSPIRE].

[55] The Sage developers, SageMath, the Sage Mathematics Software System, version 9.1, https://www.sagemath.org (2020).

[56] I. Connell, Elliptic curve handbook, https://webs.ucm.es/BUCM/mat/doc8354.pdf. 